\title{
A New Pd-catalyzed Cascade Reaction for the Synthesis of Strained Aromatic Polycycles
}

Gaëlle Blond, Christophe Bour, Bahaâ Salem, and Jean Suffert*

Laboratoire de Pharmacochimie de la Communication Cellulaire (UMR 7175 CNRS/ULP), Faculté de Pharmacie, 64 route du Rhin 67401 ILLKIRCH-Cedex, France 


\section{Table of Contents}

General Information: S2

Characterization data for 1-98: S3-S33

Spectra for $10,11,22,23,24,25,26,27,28,34,35,36,37,38,39,40,74,75,76,80,81,83$, 84, 85 : S34-S68

\section{General Information.}

Reactions were run under an atmosphere of argon in oven-dried glassware using a standard syringe, cannula and septa apparatus. $\mathrm{Et}_{2} \mathrm{O}$ and $\mathrm{THF}$ were distilled from sodium benzophenone. Benzene and DMF were distilled from $\mathrm{CaH}_{2}, \mathrm{CH}_{2} \mathrm{Cl}_{2}$ was distilled from $\mathrm{P}_{2} \mathrm{O}_{5}$. $\mathrm{EtN}_{3}$ and $i-\mathrm{Pr}_{2} \mathrm{NH}$ were distilled from $\mathrm{KOH}$. Crude products were purified by flash column chromatography on Merck 230-400 mesh silica gel. Analytical TLC, was carried out on Merck (Kieselgel 60F-254) silica gel plates. ${ }^{1} \mathrm{H}$ spectra were recorded at 200 or $300 \mathrm{MHz}$ using the residual solvent signal as internal reference of $\mathrm{CDCl}_{3}, 7.26 \mathrm{ppm}$. Chemical shifts are quoted in ppm, coupling constants $(J)$ are given in Hz. The following abbreviations are used to describe peak patterns when appropriate: s (singlet), d (doublet), $\mathrm{t}$ (triplet), q (quartet), quint (quintet), m (multiplet), ap (apparent), br (broad). ${ }^{13} \mathrm{C}$ NMR spectra were recorded at 50 or $75 \mathrm{MHz}$ at ambient temperature in $\mathrm{CDCl}_{3}$ at $77.0 \mathrm{ppm}$ as internal reference. Multiplicities were determined in some cases by Jmod pulse sequence or by DEPT pulse sequence. Melting points were determined with a glass capillary apparatus and are uncorrected. I.R. were determined with a Perkin-Elmer 2000 apparatus. Mass spectra were obtained from 6 different apparatus, some are from the Service de spectrométrie de Masse of the Chemistry Institute of Strasbourg (France). Apparatus 1: GC-MS, Agilent 5975, EI, simple quadripole, apparatus 2 : HRMS, Bruker, MicroQTof, ESI, tandem Quadripole-Tof, apparatus 3: MS, Bruker, HCT ultra, APPI or ESI, IT, apparatus 4 : MS, Agilent, MSD 1100, ESI, Q, apparatus 5 : MS, Mariner, Applied Bio-System Perkin Elmer, ESI, Tof, apparatus 6 : Bruker, Esquire HCT plus TM, ESI, IT. $n$-BuLi was titrated using pivaloyl $o$-toluidine following the Suffert's method. ${ }^{1}$ Microwave irradiations have been performed using BIOTAGE Smith Initiator. All the reactions were carried out under argon atmosphere in microwaves tubes. $\mathrm{Pd}\left(\mathrm{PPh}_{3}\right)_{4}$ was prepared from $\mathrm{PdCl}_{2}$. Commercially available materials were used without further purification.

\footnotetext{
${ }^{1}$ Suffert, J. J. Org. Chem. 1989, 54, 509-510
} 


\section{Synthesis of 1 from 57}
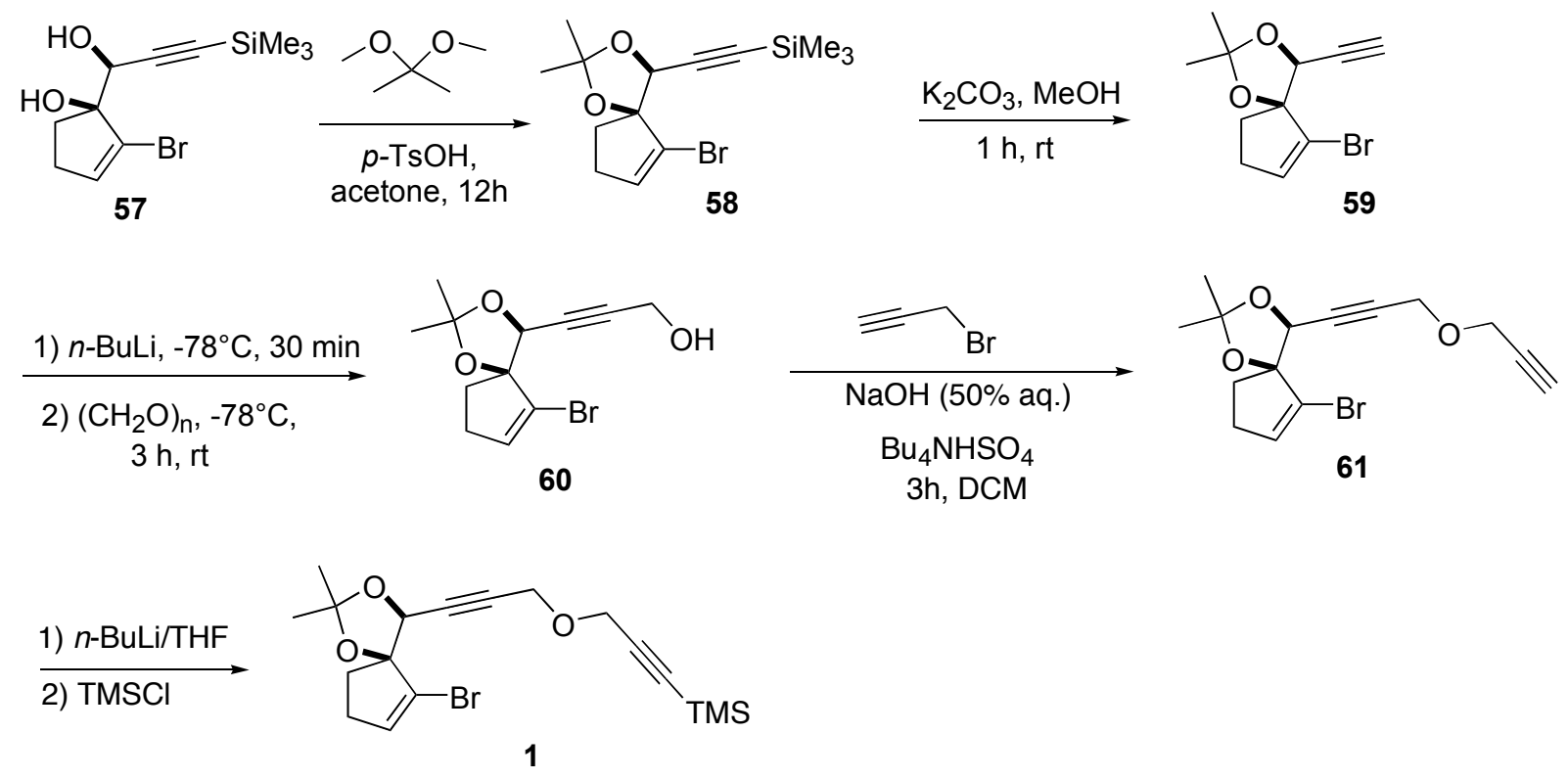

$\{[(4 \mathrm{~S} *, 5 \mathrm{~S} *)-6-$ bromo-2,2-dimethyl-1,3-dioxaspiro[4.4]non-6-en-4-yl]ethynyl\}(trimethyl)si lane (58)

A solution of diol anti $\mathbf{5 7 ^ { 2 }}$ (500 mg, $1.73 \mathrm{mmol}, 1$ eq.), 2,2-dimethoxypropane (6 mL, 49.0 mmol, 28 eq.), and $p$-TsOH (50 mg, $0.17 \mathrm{mmol}, 0.1$ eq.) in acetone (15 mL) was stirred during $12 \mathrm{~h}$ at room temperature. Then the mixture was quenched by addition of a saturated solution of $\mathrm{NaHCO}_{3}(50 \mathrm{~mL})$. After extraction with $\mathrm{Et}_{2} \mathrm{O}$, the combined organic layers were washed with water, brine, dried over $\mathrm{MgSO}_{4}$ and concentrated in vacuo. The crude product was purified by chromatography on silica gel (elution with hexane/Et ${ }_{2} \mathrm{O}: 80 / 20$ ) to give 500 $\mathrm{mg}(88 \%)$ of the product $\mathbf{5 8}$ as yellow oil.

$\mathbf{R f}=0.78$ (hexane/Et $\left.{ }_{2} \mathrm{O}: 80 / 20\right) .{ }^{1} \mathbf{H}$ NMR $\left(300 \mathrm{MHz}, \mathrm{CDCl}_{3}\right) \delta 6.11(\mathrm{t}, 1 \mathrm{H}, J=2.5 \mathrm{~Hz}), 4.78$ $(\mathrm{s}, 1 \mathrm{H}), 2.53-2.09(\mathrm{~m}, 4 \mathrm{H}), 1.65(\mathrm{~s}, 3 \mathrm{H}), 1.37(\mathrm{~s}, 3 \mathrm{H}), 0.16(\mathrm{~s}, 9 \mathrm{H}) .{ }^{13} \mathbf{C}$ NMR $(75 \mathrm{MHz}$, $\left.\mathrm{CDCl}_{3}\right) \delta 135.4,124.0,109.9,98.3,94.6,94.2,73.9,35.3,29.5,26.9,25.7,-0.5$. IR (FTIR, film) $: v=2962,2857,2177,1617,1454,1378,1326,1253,1212,1137,1097,1061,1026$, $973,942,849,760,701,649,518 \mathrm{~cm}^{-1}$.

\section{$(4 \mathrm{~S} *, 5 \mathrm{~S} *$ )-6-bromo-4-ethynyl-2,2-dimethyl-1,3-dioxaspiro[4.4]non-6-ene (59)}

A solution of compound $\mathbf{5 8}$ (500 mg, $1.52 \mathrm{mmol}, 1$ eq.) and $\mathrm{K}_{2} \mathrm{CO}_{3}$ (336 mg, $2.43 \mathrm{mmol}, 1.6$ eq.) in $\mathrm{MeOH}(10 \mathrm{~mL})$ was stirred during $1 \mathrm{~h}$ at $\mathrm{rt}$. Then the mixture was quenched by addition of a saturated solution of $\mathrm{NH}_{4} \mathrm{Cl}$. After extraction with AcOEt, the combined organic layers were washed with water, brine, dried over $\mathrm{MgSO}_{4}$ and concentrated in vacuo. The crude product was purified by chromatography on silica gel (elution with hexane/Et ${ }_{2} \mathrm{O}: 80 / 20$ ) to give $350 \mathrm{mg}(90 \%)$ of the product $\mathbf{5 9}$ as a white powder.

$\mathbf{R f}=0.63$ (hexane/Et $\left.{ }_{2} \mathrm{O}: 70 / 30\right) .{ }^{1} \mathbf{H}$ NMR $\left(300 \mathrm{MHz}, \mathrm{CDCl}_{3}\right) \delta 6.16($ br s, $1 \mathrm{H}), 4.81(\mathrm{~d}, 1 \mathrm{H}$, $J=2.3 \mathrm{~Hz}), 2.59(\mathrm{~d}, 1 \mathrm{H}, J=2.3 \mathrm{~Hz}), 2.53-2.11(\mathrm{~m}, 4 \mathrm{H}), 1.67(\mathrm{~s}, 3 \mathrm{H}), 1.40(\mathrm{~s}, 3 \mathrm{H}) .{ }^{\mathbf{1 3}} \mathbf{C} \mathbf{~ N M R}$ $\left(75 \mathrm{MHz}, \mathrm{CDCl}_{3}\right) \delta: 136.2,128.6,110.2,94.0,77.3,77.0,73.4,35.2,29.6,26.8,25.7$. IR (FTIR, film): $v=3238,2983,2928,2854,2120,1620,1433,1480,1323,1257,1212,1121$, 
$1063,934,879,847,814,734,695 \mathrm{~cm}^{-1}$.

3-[(4S *,5S*)-6-bromo-2,2-dimethyl-1,3-dioxaspiro[4.4]non-6-en-4-yl]prop-2-yn-1-ol (60)

To a solution of compound 59 (300 $\mathrm{mg}, 1.17 \mathrm{mmol}, 1$ eq.) in THF (10 mL) was added dropwise $n$-butyllithium ( $1 \mathrm{~mL}, 1.23 \mathrm{mmol}, 1.05$ eq.) at $-78^{\circ} \mathrm{C}$. The resulting solution was stirred for $30 \mathrm{~min}$ at $-78^{\circ} \mathrm{C}$ and paraformaldehyde $(53 \mathrm{mg}, 1.75 \mathrm{mmol}, 1.5 \mathrm{eq}$.) was then added. The reaction mixture was kept at $-78^{\circ} \mathrm{C}$ for $1 \mathrm{~h}$ and then allowed to warm up to rt for 3 $\mathrm{h}$ before addition of a saturated solution of $\mathrm{NaHCO}_{3}$. After extraction with $\mathrm{Et}_{2} \mathrm{O}$, the combined organic layers were washed with water, brine, dried over $\mathrm{Na}_{2} \mathrm{SO}_{4}$ and concentrated in vacuo. The crude product was purified by chromatography on silica gel (elution with hexane $\left./ \mathrm{Et}_{2} \mathrm{O}: 50 / 50\right)$ to afford $168 \mathrm{mg}(50 \%)$ of the product 60 as a colourless oil.

$\mathbf{R f}=0.18$ (hexane/Et $\left.{ }_{2} \mathrm{O}: 50 / 50\right) .{ }^{1} \mathbf{H}$ NMR $\left(300 \mathrm{MHz}, \mathrm{CDCl}_{3}\right) \delta 6.12($ br s, $1 \mathrm{H}), 4.81$ (br s, $1 \mathrm{H}), 4.28$ (br s, 2H), 2.56-2.10 (m, 4H), $1.62(\mathrm{~s}, 3 \mathrm{H}), 1.37(\mathrm{~s}, 3 \mathrm{H}) .{ }^{13} \mathrm{C}$ NMR $(75 \mathrm{MHz}$, $\left.\mathrm{CDCl}_{3}\right) \delta 136.2,123.4,109.9,93.9,87.4,78.8,73.3,50.9,35.1,29.5,26.8,25.6$. IR (FTIR, film): $v=3425,2985,2934,2858,1617,1451,1379,1256,1213,1169,1141,1059,1024$ $\mathrm{cm}^{-1}$.

$\left(4 S^{*}, 5 \mathrm{~S} *\right)$-6-bromo-2,2-dimethyl-4-[3-(prop-2-ynyloxy)prop-1-ynyl]-1,3-dioxaspiro[4.4]n on-6-ene (61)

To a solution of compound 60 (156 mg, $0.543 \mathrm{mmol}, 1$ eq.) in $\mathrm{CH}_{2} \mathrm{Cl}_{2}$ (3 mL) was added propargyl bromine (194 mg, $1.74 \mathrm{mmol}, 3.2$ eq., $80 \%$ in toluene), $n \mathrm{Bu}_{4} \mathrm{NHSO}_{4}(18 \mathrm{mg}, 0.05$ mmol, 0.1 eq.) and $1 \mathrm{~mL}$ of solution of $\mathrm{NaOH}(50 \%$ aq. $)$. After $3 \mathrm{~h}$ at RT, the produc twas extracted with $\mathrm{CH}_{2} \mathrm{Cl}_{2}$ and the combined organic layers were washed with brine and dried over $\mathrm{Na}_{2} \mathrm{SO}_{4}$ and concentrated in vacuo. The crude product was purified by chromatography on silica gel (elution with hexane/ $\left.\mathrm{Et}_{2} \mathrm{O}: 80 / 20\right)$ to afford $160 \mathrm{mg}(90 \%)$ of the product 61 as a colourless oil.

$\mathbf{R f}=0.18$ (hexane/Et $\left.{ }_{2} \mathrm{O}: 50 / 50\right) .{ }^{1} \mathbf{H}$ NMR $\left(300 \mathrm{MHz}, \mathrm{CDCl}_{3}\right) \delta 6.15($ br s, $1 \mathrm{H}), 4.85(\mathrm{~d}, 1 \mathrm{H})$, 4.33 (br s, 2H), 4.27 (s, 2H), $2.44(\mathrm{se}, 1 \mathrm{H}), 2.55-2.11(\mathrm{~m}, 4 \mathrm{H}), 1.66(\mathrm{~s}, 3 \mathrm{H}), 1.40(\mathrm{~s}, 3 \mathrm{H}) .{ }^{13} \mathrm{C}$ NMR $\left(75 \mathrm{MHz}, \mathrm{CDCl}_{3}\right) \delta 136.1,123.7,110.0,93.9,84.3,80.1,78.9,74.9,73.6,56.6,56.4$, 35.1, 29.6, 26.8, 25.7. IR (FTIR, film): $v=3288,2985,2935,2856,2362,2118,1616,1445$, $1379,1352,1256,1212,1167,1138,1084,1064,1035,917,934,891,850,759,734,669$, $519 \mathrm{~cm}^{-1}$.

[3-(\{3-[(4S *,5S *)-6-bromo-2,2-dimethyl-1,3-dioxaspiro[4-4]non-6-en-4-yl]prop-2ynyl $\}$ ox y)prop-1-ynyl](trimethyl)silane (1)

To a solution of compound 61 (126 mg, $0.387 \mathrm{mmol}, 1$ eq.) in anhydrous THF (7 mL) at $78^{\circ} \mathrm{C}$, was slowly added $n$-BuLi $(0.35 \mathrm{~mL}, 0.407 \mathrm{mmol}, 1.05$ eq. $)$. After $1 \mathrm{~h}$ at $-78^{\circ} \mathrm{C}$, TMSCl (46 mg, $0.422 \mathrm{mmol}, 1.09$ eq.) was added and the reaction mixture was kept during $2 \mathrm{~h}$ at $78^{\circ} \mathrm{C}$. A satured aqueous solution of $\mathrm{NaHCO}_{3}$ was then added in the mixture and the product extracted with $\mathrm{Et}_{2} \mathrm{O}$. The combined organic layers were washed with water, brine, dried over $\mathrm{Na}_{2} \mathrm{SO}_{4}$ and concentrated in vacuo. The crude product was purified by chromatography on silica gel (elution with hexane/Et $\left.{ }_{2} \mathrm{O}: 80 / 20\right)$ to afford $110 \mathrm{mg}(72 \%)$ of pure 1 as a brown oil.

$\mathbf{R f}=0.67\left(\right.$ hexane/Et $\left.{ }_{2} \mathrm{O}: 60 / 40\right) . \mathbf{N M R}^{1} \mathbf{H}\left(300 \mathrm{MHz}, \mathrm{CDCl}_{3}\right) \delta 6.13($ br s, $1 \mathrm{H}), 4.83(\mathrm{~d}, 1 \mathrm{H})$, $4.30($ br s, 2H), 4.25 (s, 2H), 2.55-2.10 (m, 4H), $1.64(\mathrm{~s}, 3 \mathrm{H}), 1.39$ (s, 3H), 0.17 (s, 9H). NMR ${ }^{13} \mathrm{C}\left(75 \mathrm{MHz}, \mathrm{CDCl}_{3}\right) \delta 136.1,123.7,110.0,100.5,93.9,84.5,79.9,73.6,57.2,56.5$, $35.1,29.5,26.8,25.7,-0.3$. IR (FTIR, film): $v=2959,2855,2172,1654,1617,1442,1379$, 
$1254,1212,1167,1138,1085,1001,891,847,761 \mathrm{~cm}^{-1}$.

\section{Synthesis of 4 from propargylic alcohol}
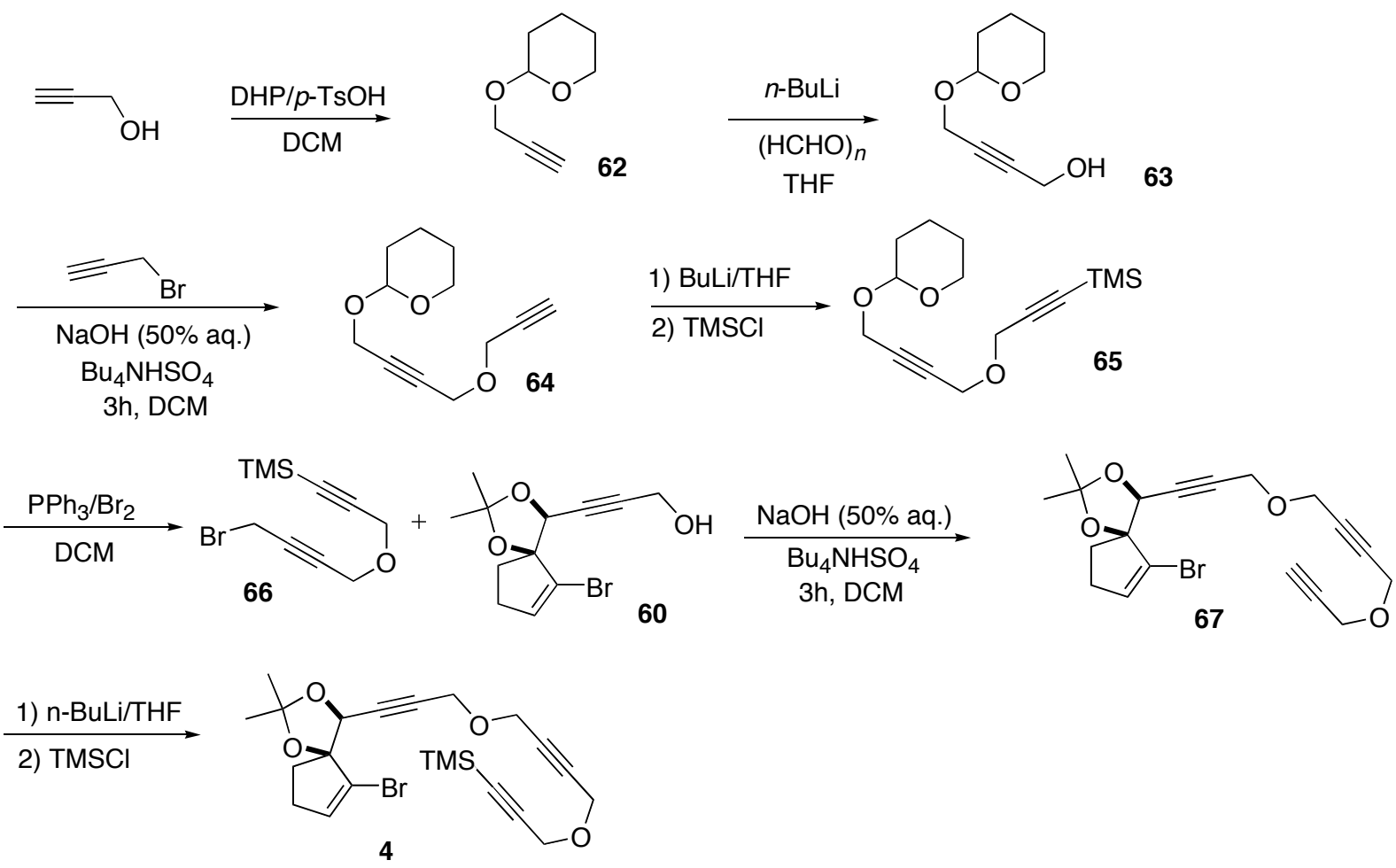

\section{2-(prop-2-ynyloxy)tetrahydro-2H-pyran (62)}

To a solution of propargylic alcohol $(20 \mathrm{~g}, 0.357 \mathrm{~mol}$, 1eq. $)$ in $\mathrm{CH}_{2} \mathrm{Cl}_{2}(20 \mathrm{~mL})$, at $0^{\circ} \mathrm{C}$ was slowly added dihydropyrane ( $45 \mathrm{~g}, 0.535 \mathrm{~mol}, 1 \mathrm{eq}$.) with a catalytic quantity of $p$-TsOH. After one night at room temperature, a saturated aqueous solution of $\mathrm{NaHCO}_{3}$ was added, and the usual aqueous treatment was realised. The crude mixture was distilled $\left(65^{\circ} \mathrm{C}, 20 \mathrm{mmHg}\right)$ to afford 62 (45 g, $90 \%)$ as a colorless oil.

$\mathbf{R f}=0.59$ (hexane/ $\left.\mathrm{Et}_{2} \mathrm{O}: 50 / 50\right) .{ }^{1} \mathbf{H}$ NMR $\left(200 \mathrm{MHz}, \mathrm{CDCl}_{3}\right) \delta 4.78(\mathrm{br} \mathrm{s}, 1 \mathrm{H}), 4.29(\mathrm{dd}, 1 \mathrm{H}$, $J=15.1 \mathrm{~Hz}, J=2.4 \mathrm{~Hz}), 4.18(\mathrm{dd}, 1 \mathrm{H}, J=15.1 \mathrm{~Hz}, J=2.4 \mathrm{~Hz}), 3.90-3.70(\mathrm{~m}, 1 \mathrm{H}), 3.59-3.43$ (m, $1 \mathrm{H}), 2.40(\mathrm{t}, 1 \mathrm{H}, J=2.4 \mathrm{~Hz}), 1.90-1.40(\mathrm{~m}, 6 \mathrm{H})$.

\section{4-(tetrahydro-2H-pyran-2-yloxy)but-2-yn-1-ol (63)}

To a solution of $62\left(4.6 \mathrm{~g}, 33.0 \mathrm{mmol}, 1\right.$ eq.) in anhydrous THF at $-78^{\circ} \mathrm{C}$ was slowly added $n$-BuLi (30 mL, $34.5 \mathrm{mmol}, 1.05$ eq.). After $1 \mathrm{~h}$ at $-78^{\circ} \mathrm{C}$, paraformaldehyde $(1.035 \mathrm{~g}, 34.5$ mmol, 1.05 eq.) was added. After one night at room temperature, the reaction mixture was extracted with $\mathrm{Et}_{2} \mathrm{O}$, then organic phase was washed with brine, dried over $\mathrm{Na}_{2} \mathrm{SO}_{4}$, filtrated and concentrated in vacuo. No purification was necessary to isolate product 63 ( $5 \mathrm{~g}, 89 \%)$ as pure yellow oil.

$\mathbf{R f}=0.17$ (hexane/Et $\left.{ }_{2} \mathrm{O}: 50 / 50\right) .{ }^{1} \mathbf{H}$ NMR $\left(300 \mathrm{MHz}, \mathrm{CDCl}_{3}\right) \delta 4.80$ (br s, $\left.1 \mathrm{H}\right), 4.40-4.21$ (m, 4H), 3.91-3.72 (m, 1H), 3.59-3.45 (m, 1H), 2.49 (br s, 1H), 1.92-1.48 (m, 6H). ${ }^{13} \mathbf{C ~ N M R}$ $\left(75 \mathrm{MHz}, \mathrm{CDCl}_{3}\right) \delta 96.8,84.4,81.4,61.9,54.3,50.9,30.1,25.2,18.9$.

2-\{[4-(prop-2-ynyloxy)but-2-ynyl]oxy\}tetrahydro-2H-pyran (64) 
Compound 64 was prepared following the same procedure than for the preparation of $\mathbf{6 1}$, starting from compound 63 (4.5 g, $26.4 \mathrm{mmol}, 1 \mathrm{eq}$ ), propargylic alcohol (14 g, $92.0 \mathrm{mmol}$, 3.5 eq., $80 \%$ in toluene), $n \mathrm{Bu}_{4} \mathrm{NHSO}_{4}(892 \mathrm{mg}, 2.6 \mathrm{mmol}, 0.1$ eq.) and $15 \mathrm{~mL}$ of solution of $\mathrm{NaOH}\left(50 \%\right.$ aq.) in $\mathrm{CH}_{2} \mathrm{Cl}_{2}(50 \mathrm{~mL})$. Aqueous treatment and chromatography on silica gel (elution with hexane/Et $\left.\mathrm{Et}_{2} \mathrm{O}: 80 / 20\right)$ afford $3.9 \mathrm{~g}(70 \%)$ of the product 64 as a viscous oil.

$\mathbf{R f}=0.35$ (hexane/Et $\left.{ }_{2} \mathrm{O}: 50 / 50\right) .{ }^{1} \mathbf{H}$ NMR $\left(300 \mathrm{MHz}, \mathrm{CDCl}_{3}\right) \delta 4.81-4.76(\mathrm{~m}, 1 \mathrm{H}), 4.33-4.21$ $(\mathrm{m}, 6 \mathrm{H}), 3.91-3.72(\mathrm{~m}, 1 \mathrm{H}), 3.59-3.45(\mathrm{~m}, 1 \mathrm{H}), 2.45(\mathrm{t}, 1 \mathrm{H}, J=2.4 \mathrm{~Hz}), 1.92-1.48(\mathrm{~m}, 6 \mathrm{H})$. ${ }^{13}$ C NMR $\left(75 \mathrm{MHz}, \mathrm{CDCl}_{3}\right) \delta 96.8,83.0,80.9,78.9,74.9,61.9,56.8,56.4,54.2,30.2$, $25.3,19.0$.

\section{Trimethyl(3-\{[4-(tetrahydro-2H-pyran-2-yloxy)but-2-ynyl]oxy\}prop-1-ynyl)silane (65)}

Compound 65 was prepared following the same procedure than for the preparation of $\mathbf{1}$, starting from compound 64 (3.5 g, $16.7 \mathrm{mmol}, 1$ eq.), $n$-BuLi (13 mL, $17.6 \mathrm{mmol}, 1.05$ eq.) and TMSCl (2 g, $18.2 \mathrm{mmol}, 1.09$ eq.) in anhydrous THF $(50 \mathrm{~mL})$. After $2 \mathrm{~h}$ of reaction, a saturated aqueous solution of $\mathrm{NaHCO}_{3}$ was added, and the usual aqueous treatment was applied. No purification was necessary to obtain pure product $65(4.7 \mathrm{~g}, 100 \%)$ as brown oil.

$\mathbf{R f}=0.55$ (hexane/Et $\left.{ }_{2} \mathrm{O}: 50 / 50\right) .{ }^{1} \mathbf{H}$ NMR $\left(300 \mathrm{MHz}, \mathrm{CDCl}_{3}\right) \delta 4.81-4.76(\mathrm{~m}, 1 \mathrm{H}), 4.33-4.19$ $(\mathrm{m}, 6 \mathrm{H}), 3.91-3.75(\mathrm{~m}, 1 \mathrm{H}), 3.59-3.45(\mathrm{~m}, 1 \mathrm{H}), 1.92-1.48(\mathrm{~m}, 6 \mathrm{H}), 0.16(\mathrm{~s}, 9 \mathrm{H}) .{ }^{13} \mathbf{C}$ NMR $\left(50 \mathrm{MHz}, \mathrm{CDCl}_{3}\right) \delta 100.5,96.8,92.0,82.9,81.1,61.9,57.3,56.8,54.2,30.2,25.3,19.0,-0.3$.

\section{$\{3-[(4-b r o m o b u t-2-y n y l) o x y] p r o p-1-y n y l\}($ trimethyl)silane (66)}

To a solution of triphenylphosphine $(6.4 \mathrm{~g}, 24.2 \mathrm{mmol}, 1.7$ eq. $)$ in $\mathrm{CH}_{2} \mathrm{Cl}_{2}(50 \mathrm{~mL})$ at $-10^{\circ} \mathrm{C}$ was added slowly $\mathrm{Br}_{2}(3.9 \mathrm{~g}, 24.2 \mathrm{mmol}, 1.7 \mathrm{eq}$. $)$. After $3 \mathrm{~h} 30$ at $-10^{\circ} \mathrm{C}$, a solution of $65(4 \mathrm{~g}$, $14.3 \mathrm{mmol}, 1$ eq.) in $\mathrm{CH}_{2} \mathrm{Cl}_{2}(25 \mathrm{~mL})$ was added at $-10^{\circ} \mathrm{C}$. After $2 \mathrm{~h}$ at $0^{\circ} \mathrm{C}$ and $2 \mathrm{~h}$ at room temperature, a saturated aqueous solution of $\mathrm{NaHCO}_{3}$ was added and the combined extracts were washed with brine and dried over $\mathrm{Na}_{2} \mathrm{SO}_{4}$. Chromatography on silica gel (elution with hexane/Et $\left.{ }_{2} \mathrm{O}: 80 / 20\right)$ afford $3.4 \mathrm{~g}(92 \%)$ of the product 66 as a brown oil.

$\mathbf{R f}=0.68$ (hexane/Et $\left.\mathrm{Et}_{2} \mathrm{O}: 50 / 50\right) . \mathbf{N M R}^{1} \mathbf{H}\left(200 \mathrm{MHz}, \mathrm{CDCl}_{3}\right) \delta 4.30(\mathrm{t}, 2 \mathrm{H}, J=2.0 \mathrm{~Hz}), 4.21$ $(\mathrm{t}, 2 \mathrm{H}), 3.95(\mathrm{t}, 2 \mathrm{H}, J=2.0 \mathrm{~Hz}), 0.17(\mathrm{~s}, 9 \mathrm{H}) . \mathbf{N M R}{ }^{13} \mathbf{C}\left(75 \mathrm{MHz}, \mathrm{CDCl}_{3}\right) \delta 100.4,92.3,82.2$, $81.8,57.5,56.5,13.9,-0.2$.

(4S *,5S *)-6-bromo-2,2-dimethyl-4-(3-\{[4-(prop-2-ynyloxy)but-2-ynyl]oxy\}prop-1-ynyl)-1 , 3-dioxaspiro[4.5]dec-6-ene (67)

To a solution of compound $\mathbf{6 0}$ (130 mg, $0.497 \mathrm{mmol}, 1$ eq.) in $\mathrm{CH}_{2} \mathrm{Cl}_{2}$ (6 mL) was added compound 66 (450 mg, $1.738 \mathrm{mmol}, 3,5$ eq.), $n-\mathrm{Bu}_{4} \mathrm{NHSO}_{4}(18 \mathrm{mg}, 0.054 \mathrm{mmol}, 0.1$ eq.) and $2 \mathrm{~mL}$ of solution of $\mathrm{NaOH}(50 \%$ aq.). After $2 \mathrm{~h}$ at RT $30 \mathrm{~mL}$ of water was added and the product was extracted with $\mathrm{CH}_{2} \mathrm{Cl}_{2}(25 \mathrm{~mL})$ and the combined organic layers were washed with brine and dried over $\mathrm{Na}_{2} \mathrm{SO}_{4}$ and concentrated in vacuo. The crude product was purified by chromatography on silica gel (elution with hexane/Et $\left.{ }_{2} \mathrm{O}: 85 / 15\right)$ to afford $110 \mathrm{mg}(56 \%)$ of the compound 67 as a yellow oil.

$\mathbf{R f}=0.49$ (hexane/Et $\left.{ }_{2} \mathrm{O}: 50 / 50\right) . \mathbf{N M R}^{1} \mathbf{H}\left(200 \mathrm{MHz}, \mathrm{CDCl}_{3}\right) \delta 6.10$ (br t, $\left.1 \mathrm{H}\right), 4.81(\mathrm{t}, 1 \mathrm{H}, J$ $=1.7 \mathrm{~Hz}), 4.27(\mathrm{~s}, 6 \mathrm{H}), 4.21(\mathrm{~d}, 2 \mathrm{H}, J=2.5 \mathrm{~Hz}), 2.44(\mathrm{t}, 1 \mathrm{H}, J=2.5 \mathrm{~Hz}), 2.41-2.08(\mathrm{~m}, 4 \mathrm{H})$, $1.62(\mathrm{~s}, 3 \mathrm{H}), 1.36(\mathrm{~s}, 3 \mathrm{H}) . \mathbf{N M R}{ }^{13} \mathbf{C}\left(50 \mathrm{MHz}, \mathrm{CDCl}_{3}\right) \delta 136.0,123.5,109.9,93.8,84.2,82.1$, $81.8,80.0,78.7,75.0,73.4,56.5,56.3,35.0,29.5,26.7,25.5$. IR (FTIR, film) : $v=3286$, 2984, 2936, 2855, 2362, 2251, 2117, 2007, 1711, 1616, 1443, 1378, 1349, 1256, 1212, 1167 , 
$1124,1069,1034,977,930,892,850,811,668 \mathrm{~cm}^{-1}$.

(3-\{[4-(\{3-[(4S*,5S*)-6-bromo-2,2-dimethyl-1,3-dioxaspiro[4.4]non-6-en-4-yl]prop-2ynyl \}oxy)but-2-ynyl]oxy\}prop-1-ynyl)(trimethyl)silane (4)

Compound 4 was prepared following the same procedure than for the preparation of $\mathbf{1}$, starting from compound 67 (100 mg, $0.254 \mathrm{mmol}, 1$ eq. $), n$-BuLi $(0.3 \mathrm{~mL}, 0.267 \mathrm{mmol}, 1.05$ eq.) and TMSCl (30 mg, $0.277 \mathrm{mmol}, 1.09$ eq.) in anhydrous THF (7 mL). After an aqueous treatment and chromatography on silica gel (elution with hexane/Et $\left.{ }_{2} \mathrm{O}: 85 / 15\right), 50 \mathrm{mg}(42 \%)$ of product 4 was obtained as a yellow oil.

$\mathbf{R f}=0.66$ (hexane / $\left.\mathrm{Et}_{2} \mathrm{O}: 50 / 50\right) . \mathbf{N M R}^{1} \mathbf{H}\left(200 \mathrm{MHz}, \mathrm{CDCl}_{3}\right) \delta 6.14(\mathrm{~m}, 1 \mathrm{H}), 4.84(\mathrm{~m}, 1 \mathrm{H})$, 4.39-4.27 (m, 6H), 4.23 (s, 2H), 2.55-2.17 (m, 4H), 1.65 (s, 3H), 1.39 (s, 3H), 0.18 (s, 9H). NMR ${ }^{13} \mathbf{C}\left(50 \mathrm{MHz}, \mathrm{CDCl}_{3}\right) \delta 136.1,123.7,110.0,100.4,93.9,92.1,84.3,82.1,82.1,80.1$, 73.6, 57.3, 56.7, 56.6, 35.1, 29.6, 26.9, 25.6, 0.3. IR (film) : $v=2953,2930,2856,2355$, $2340,2175,1727,1667,1607,1442,1375,1345,1248,1211,1121,1076,1001,889,844$, $761 \mathrm{~cm}^{-1}$.

\section{Synthesis of 10 from 30}
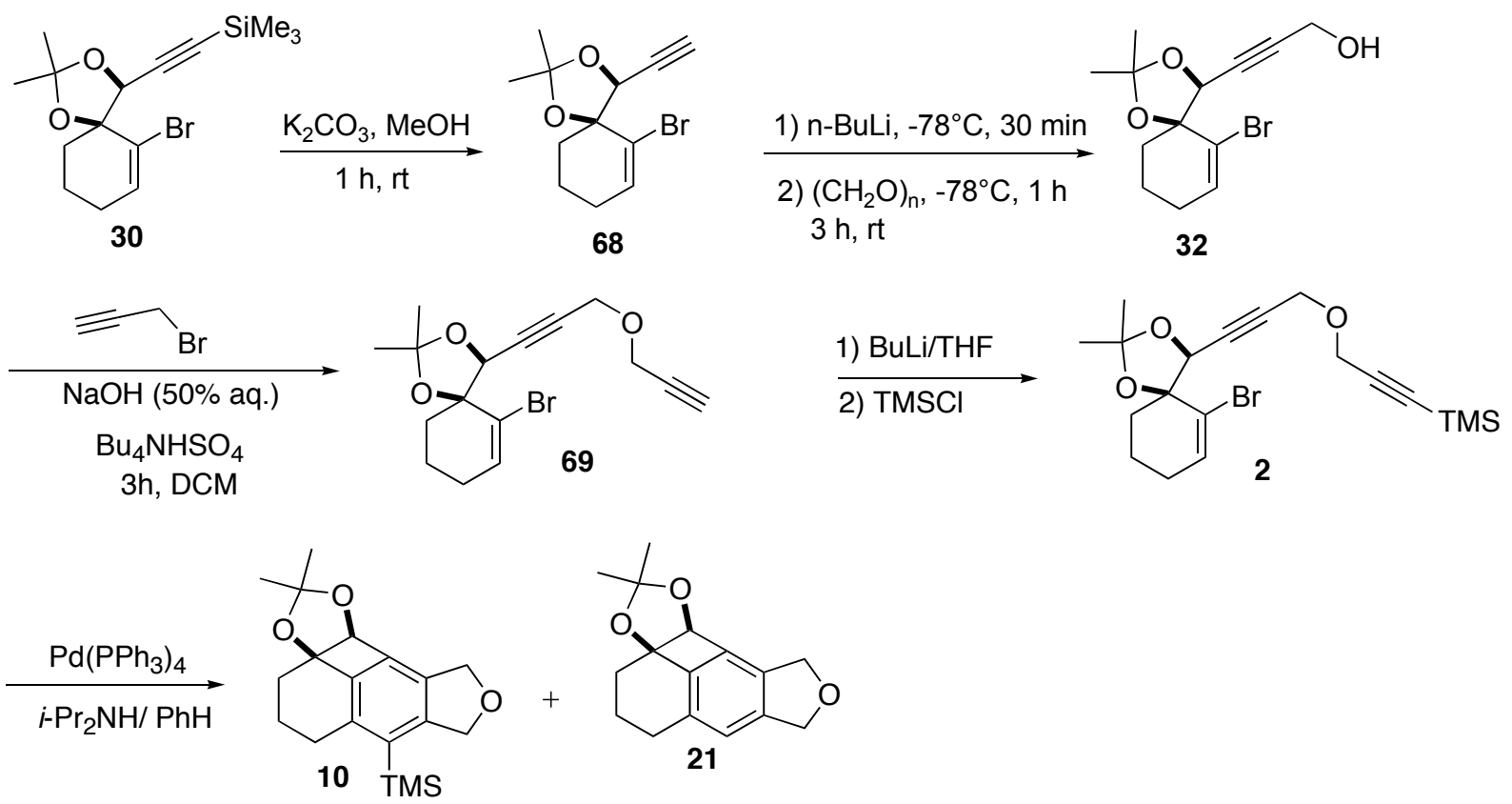

\section{$\left(4 S^{*}, 5 S^{*}\right)$-6-bromo-4-ethynyl-2,2-dimethyl-1,3-dioxaspiro[4.5] dec-6-ene (68)}

A solution of compound 30 ( $2 \mathrm{~g}, 5.83 \mathrm{mmol}, 1$ eq.) and $\mathrm{K}_{2} \mathrm{CO}_{3}$ (806 mg, $5.83 \mathrm{mmol}, 1$ eq.) in $\mathrm{MeOH}(29 \mathrm{~mL})$ was stirred during $1 \mathrm{~h}$ at $\mathrm{rt}$. Then the mixture was quenched by addition of a saturated solution of $\mathrm{NH}_{4} \mathrm{Cl}(30 \mathrm{~mL})$. After extraction with AcOEt, the combined organic phases were washed with water, brine, dried over $\mathrm{MgSO}_{4}$ and concentrated in vacuo. The crude product was purified by chromatography on silica gel (elution with heptane/AcOEt: $95 / 5)$ to give $1.57 \mathrm{~g}(99 \%)$ of $\mathbf{6 8}$ as a white powder.

$\mathbf{R f}=0.21($ heptane/AcOEt: $95 / 5) . \mathbf{M p}=64^{\circ} \mathrm{C} .{ }^{1} \mathbf{H}$ NMR $\left(200 \mathrm{MHz}, \mathrm{CDCl}_{3}\right) \delta 6.38(\mathrm{t}, J=4.2$ $\mathrm{Hz}, 1 \mathrm{H}), 4.72(\mathrm{~d}, J=2.4 \mathrm{~Hz}, 1 \mathrm{H}), 2.64(\mathrm{~d}, J=2.5 \mathrm{~Hz}, 1 \mathrm{H}), 2.16-1.79(\mathrm{~m}, 6 \mathrm{H}), 1.70(\mathrm{~s}, 3 \mathrm{H})$, $1.41(\mathrm{~s}, 3 \mathrm{H}) .{ }^{13} \mathrm{C}$ NMR $\left(75 \mathrm{MHz}, \mathrm{CDCl}_{3}\right) \delta 135.1,124.2,110.6,83.7,78.2,77.4,75.4,38.3$, 27.5, 26.3, 19.7. IR $\left(\mathrm{CHCl}_{3}\right) v=3287,2985,2939,2864,2360,2078,1456,1376,1259$, 
1210, 1167, 1124, 1068, 1021, 991, 946, 897, 875, 803, 750, 712, $520 \mathrm{~cm}^{-1}$. GC-MS (apparatus 1) : 270.0, $272.0[\mathrm{M}+\cdot]$.

\section{3 -[(4S*,5S*)-6-bromo-2,2-dimethyl-1,3-dioxaspiro[4.5]dec-6-en-4-yl]prop-2-yn-1-ol (32)}

To a solution of compound $68(1.29 \mathrm{~g}, 4.76 \mathrm{mmol}$, 1 eq.) in THF (40 mL) was added dropwise $n$-butyllithium (3.0 mL, $4.62 \mathrm{mmol}, 1.06$ eq., $1.54 \mathrm{M}$ in hexane) at $-78^{\circ} \mathrm{C}$. The resulting solution was stirred for $30 \mathrm{~min}$ at $-78^{\circ} \mathrm{C}$ and paraformaldehyde $(198 \mathrm{mg}, 6.6 \mathrm{mmol}$, 1.5 eq.) was then added. The reaction mixture was kept at $-78^{\circ} \mathrm{C}$ for $1 \mathrm{~h}$ and then allowed to warm up to $\mathrm{rt}$ for $3 \mathrm{~h}$ before addition of a saturated solution of $\mathrm{NaHCO}_{3}(20 \mathrm{~mL})$. After extraction with $\mathrm{Et}_{2} \mathrm{O}$, the combined organic layers were washed with water, brine, dried over $\mathrm{Na}_{2} \mathrm{SO}_{4}$ and concentrated in vacuo. The crude product was purified by chromatography on silica gel (elution with heptane/ $\left.\mathrm{Et}_{2} \mathrm{O}: 50 / 50\right)$ to afford $1.247 \mathrm{~g}(87 \%)$ of the product 32 as a yellow solid.

$\mathbf{R f}=0.14\left(\right.$ heptane/Et $\left.\mathrm{E}_{2} \mathrm{O}: 40 / 60\right) . \mathbf{M p}=45^{\circ} \mathrm{C} .{ }^{1} \mathbf{H}$ NMR $\left(200 \mathrm{MHz}, \mathrm{CDCl}_{3}\right) \delta 6.37(\mathrm{t}, J=4.2$ $\mathrm{Hz}, 1 \mathrm{H}), 4.73(\mathrm{t}, J=1.5 \mathrm{~Hz}, 1 \mathrm{H}), 4.33(\mathrm{dd}, J=6.1 \mathrm{~Hz}, J=1.5 \mathrm{~Hz}, 2 \mathrm{H}), 2.15-1.59(\mathrm{~m}, 7 \mathrm{H})$, $1.68(\mathrm{~s}, 3 \mathrm{H}), 1.40(\mathrm{~s}, 3 \mathrm{H}) .{ }^{13} \mathbf{C} \mathbf{N M R}\left(50 \mathrm{MHz}, \mathrm{CDCl}_{3}\right) \delta 135.2,124.2,110.5,87.5,83.6,80.0$, 75.4, 51.1, 38.2, 27.5, 26.4, 26.4, 19.7. IR $\left(\mathrm{CHCl}_{3}\right) v=3420,2938,2863,1634,1455,1378$, 1253, 1211, 1164, 1123, 1065, 1023, 988, 965, 901, 802, 710, $521 \mathrm{~cm}^{-1}$. GC-MS (apparatus 1) : $300.1,302.1[\mathrm{M}+\cdot]$.

\section{$(4 \mathrm{~S}$ *,5S *)-6-bromo-2,2-dimethyl-4-[3-(prop-2-ynyloxy)prop-1-ynyl]-1,3-dioxaspiro [4.5]dec-6-ene (69)}

Compound 69 was prepared following the same procedure than for the preparation of 61 , starting from compound 32 (180 mg, $0.60 \mathrm{mmol}, 1$ eq.), propargyl bromine (228 mg, 1.91 mmol, 3.2 eq., $80 \%$ in toluene), $n \mathrm{Bu}_{4} \mathrm{NHSO}_{4}(20 \mathrm{mg}, 0.06 \mathrm{mmol}, 0.1$ eq.) and $1 \mathrm{~mL}$ of solution of $\mathrm{NaOH}\left(50 \%\right.$ aq.) in $\mathrm{CH}_{2} \mathrm{Cl}_{2}(3 \mathrm{~mL})$. Aqueous treatment, extraction with $\mathrm{CH}_{2} \mathrm{Cl}_{2}$ $(20 \mathrm{~mL})$ and chromatography on silica gel (elution with hexane/Et $\left.{ }_{2} \mathrm{O}: 90 / 10\right)$ afford $150 \mathrm{mg}$ $(74 \%)$ of the product 69 as a yellow oil.

$\mathbf{R f}=0.75$ (hexane/Et $\left.{ }_{2} \mathrm{O}: 50 / 50\right) . \mathbf{N M R}^{1} \mathbf{H}\left(300 \mathrm{MHz}, \mathrm{CDCl}_{3}\right) \delta 6.36(\mathrm{t}, 1 \mathrm{H}, J=4.1 \mathrm{~Hz}), 4.75$ (br s, $1 \mathrm{H}), 4.33(\mathrm{se}, 2 \mathrm{H}), 4.29(\mathrm{t}, 2 \mathrm{H}, J=2.0 \mathrm{~Hz}), 2.43$ (t, $1 \mathrm{H}, J=2.0 \mathrm{~Hz}), 2.21-1.94(\mathrm{~m}$, $4 \mathrm{H}), 1.90-1.67(\mathrm{~m}, 2 \mathrm{H}), 1.68(\mathrm{~s}, 3 \mathrm{H}), 1.40(\mathrm{~s}, 3 \mathrm{H}) . \mathbf{N M R}{ }^{13} \mathbf{C}\left(75 \mathrm{MHz}, \mathrm{CDCl}_{3}\right) \delta 135.0$, $124.4,110.5,84.2,83.5,81.3,78.9,75.5,74.8,56.6,56.3,38.2,27.4,26.3,26.2$, 19.6. IR (FTIR, film): = 3286, 2986, 2940, 2861, 2118, 1633, 1447, 1378, 1255, 1211, 1164, 1122, 1080, 1039, 990, 965, 901, 806, 710, $669 \mathrm{~cm}^{-1}$. HRMS (Apparatus 5, ESI, positif ion): $\left(\mathrm{C}_{16} \mathrm{H}_{19} \mathrm{BrO}_{3} \mathrm{Na}\right)^{+}$calc. : 361.0321 , found : 361.0416 .

\section{[3-(\{3-[(4S*,5S*)-6-bromo-2,2-dimethyl-1,3-dioxaspiro[4.5]dec-6-en-4-yl]prop-2- ynyl\}oxy)prop-1-ynyl](trimethyl)silane (2)}

Compound 2 was prepared following the same procedure than for the preparation of $\mathbf{1}$, starting from compound 69 (150 mg, $0.442 \mathrm{mmol}, 1$ eq.), $n$-BuLi (0.52 mL, $0.464 \mathrm{mmol}, 1.05$ eq.) and $\mathrm{TMSCl}(51 \mathrm{mg}, 0.482 \mathrm{mmol}, 1.09$ eq.) in anhydrous THF (5 mL). After an aqueous treatment, extraction with $\mathrm{CH}_{2} \mathrm{Cl}_{2} 20 \mathrm{~mL}$ ) and chromatography on silica gel (elution with hexane/Et $\left.\mathrm{E}_{2} \mathrm{O}: 90 / 10\right), 140 \mathrm{mg}(77 \%)$ of product 2 was obtained as a yellow oil.

$\mathbf{R f}=0.38$ (hexane/Et $\left.{ }_{2} \mathrm{O}: 80 / 20\right) . \mathbf{N M R}^{1} \mathbf{H}\left(300 \mathrm{MHz}, \mathrm{CDCl}_{3}\right) \delta 6.37(\mathrm{t}, 1 \mathrm{H}, J=4.1 \mathrm{~Hz}), 4.76$ (br s, 1H), 4.33 (se, 2H), 4.29 (br s, 2H), 2.21-1.94 (m, 4H), 1.89-1.67 (m, 2H), $1.68(\mathrm{~s}, 3 \mathrm{H})$, 
$1.41(\mathrm{~s}, 3 \mathrm{H}), 0.19(\mathrm{~s}, 9 \mathrm{H}) . \mathbf{N M R}{ }^{13} \mathbf{C}\left(75 \mathrm{MHz}, \mathrm{CDCl}_{3}\right) \delta 134.9,124.4,110.4,100.6,91.9$, 84.2, 83.5, 81.1, 75.5, 57.1, 56.6, 56.3, 38.2, 27.4, 26.3, 26.2, 19.7, 0.2. IR (FTIR, film) : $v=$ $3348,2945,2861,2362,2175,1446,1378,1347,1253,1210,1164,1082,1040,1000,899$, $848,761,709 \mathrm{~cm}^{-1}$.

[(3aR*,10cS*)-2,2-dimethyl-5,6,10,10c-tetrahydro-4H,8H-furo[3",4":2',3']naphtho $\left[8^{\prime}, 1^{\prime}: 1,4,3\right]$ cyclobuta[1,2-d] $[1,3]$ dioxol-7-yl](trimethyl)silane (10) and $\left(3 \mathrm{a} R^{*}, 10 \mathrm{c} S^{*}\right)-2,2-$ dimethyl-5,6,10,10c-tetrahydro-4H, 8furo $\left[3^{\prime \prime}, 4^{\prime \prime}: 2^{\prime}, 3^{\prime}\right]$ naphtho $\left[8^{\prime}, 1^{\prime}: 1,4,3\right]$ cyclobuta[1,2d] [1,3]dioxole (21)

To a solution of compound 2 (100 mg, $0.243 \mathrm{mmol}, 1$ eq.) in anhydrous benzene ( $7 \mathrm{~mL})$ was added $\mathrm{Pd}\left(\mathrm{PPh}_{3}\right)_{4}(26 \mathrm{mg}, 0,023 \mathrm{mmol}, 0,1$ eq.) and diisopropyl amine $(3 \mathrm{~mL})$. The mixture was purged 5 min with argon and heated at $130^{\circ} \mathrm{C}$ under microwave irradiation during 20 min. The reaction mixture was concentrated in vacuo and purified by chromatography on silica gel (elution with heptane/Et $\left.{ }_{2} \mathrm{O}: 90 / 10\right)$ to afford $61 \mathrm{mg}(76 \%)$ of $\mathbf{1 0}$ as a yellow solid and $8 \mathrm{mg}(13 \%)$ of 21 as a yellow oil.

$10: \mathbf{R f}=0.38\left(\right.$ heptane $\left./ \mathrm{Et}_{2} \mathrm{O}: 80 / 20\right) . \mathbf{M p}=81^{\circ} \mathrm{C} . \mathbf{N M R}{ }^{1} \mathbf{H}\left(300 \mathrm{MHz}, \mathrm{CDCl}_{3}\right) \delta 5.27(\mathrm{~s}$, $1 \mathrm{H}), 5.07(\mathrm{~s}, 1 \mathrm{H}), 5.15-5.03(\mathrm{~m}, 3 \mathrm{H}), 3.10(\mathrm{dd}, 1 \mathrm{H}, J=17.3 \mathrm{~Hz}, J=6.4 \mathrm{~Hz}), 2.55-1.99(\mathrm{~m}$, $4 \mathrm{H}), 1.69-1.46(\mathrm{~m}, 1 \mathrm{H}), 1.51(\mathrm{~s}, 3 \mathrm{H}), 1.41(\mathrm{~s}, 3 \mathrm{H}), 0.31(\mathrm{~s}, 9 \mathrm{H}) . \mathbf{N M R}{ }^{13} \mathbf{C}\left(50 \mathrm{MHz}, \mathrm{CDCl}_{3}\right) \delta$ 149.5, 146.8, 140.5, 139.6, 133.1, 131.0, 114.6, 89.1, 83.8, 74.3, 70.6, 29.0, 28.9 , 28.2 , 28.0, 22.6, 1.0. COSY, HSQC, HMBC, NOESY. IR (FTIR, film) : $v=3377,2944,2855,1612$, 1433, 1381, 1307, 1253, 1198, 1147,1097, 1063, 1006, 919, 859, 839, $734 \mathrm{~cm}^{-1}$. GC-MS (apparatus 1) : $330.2\left[\mathrm{M}^{+\bullet}\right], 272.1,243.1,73.1$.

$21: \mathbf{R f}=0.28\left(\right.$ heptane $\left./ \mathrm{Et}_{2} \mathrm{O}: 80 / 20\right) . \mathbf{N M R}{ }^{1} \mathbf{H}\left(200 \mathrm{MHz}, \mathrm{CDCl}_{3}\right) \delta 6.94(\mathrm{~s}, 1 \mathrm{H}), 5.27(\mathrm{~s}$, $1 \mathrm{H}), 5.15-4.91(\mathrm{~m}, 4 \mathrm{H}), 3.06-2.95(\mathrm{~m}, 1 \mathrm{H}), 2.51-1.99(\mathrm{~m}, 4 \mathrm{H}), 1.63-1.55(\mathrm{~m}, 1 \mathrm{H}), 1.52(\mathrm{~s}$, $3 \mathrm{H}), 1.43(\mathrm{~s}, 3 \mathrm{H}) . \mathbf{N M R}{ }^{13} \mathbf{C}\left(50 \mathrm{MHz}, \mathrm{CDCl}_{3}\right) \delta 148.2,144.1,140.0,134.2,132.3,121.2$, 114.6, 88.3, 84.1, 73.2, 71.1, 29.4, 29.0, 28.2, 25.5, 22.0. MS (apparatus 3, ESI, positif ion): $259.1\left[\mathrm{MH}^{+}\right], 242.1,201.1\left[\mathrm{M}+\mathrm{K}^{+}\right]$.

\section{Synthesis of 13 from 32}<smiles>CSC#CCOCC#CCBr</smiles>

32

1) n-BuLi/THF

2) TMSCI

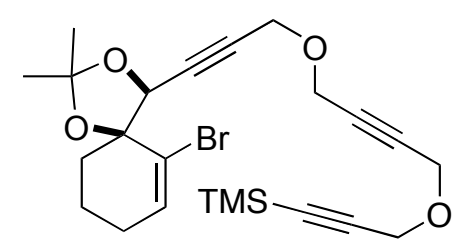

5
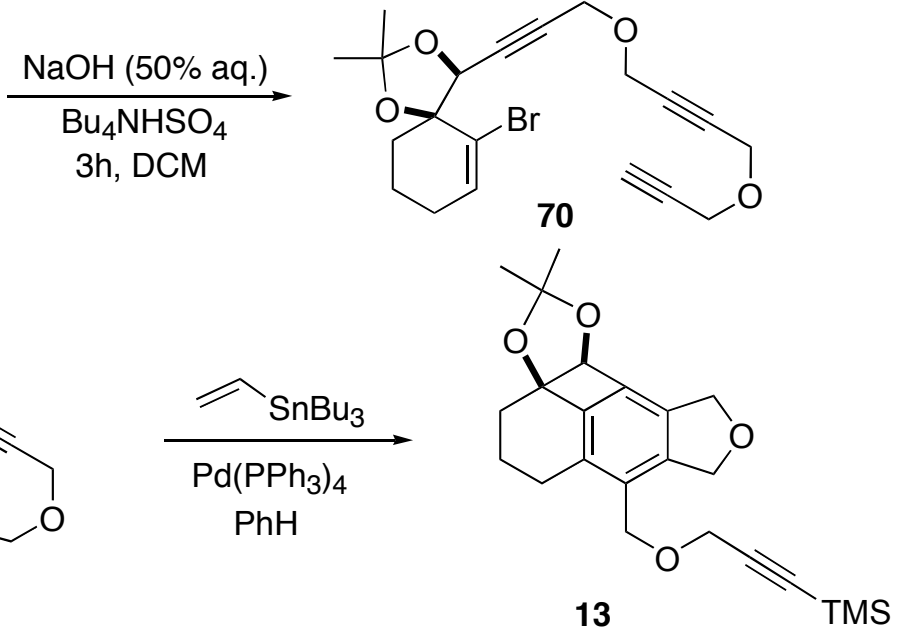

$\left(4 S^{*}, 5 \mathrm{~S} *\right)-6-$ bromo-2,2-dimethyl-4-(3-\{[4-(prop-2-ynyloxy)but-2-ynyl]oxy\}prop-1-ynyl)-1 ,3-dioxaspiro[4.5]dec-6-ene (70)

To a solution of compound $32\left(130 \mathrm{mg}, 0.430 \mathrm{mmol}, 1\right.$ eq.) in $\mathrm{CH}_{2} \mathrm{Cl}_{2}(10 \mathrm{~mL})$ was added 
compound 66 (336 mg, $1.30 \mathrm{mmol}, 3.2$ eq.), $n-\mathrm{Bu}_{4} \mathrm{NHSO}_{4}(20 \mathrm{mg}, 0.04 \mathrm{mmol}, 0.1$ eq.) and 2 $\mathrm{mL}$ of solution of $\mathrm{NaOH}(50 \%$ aq.). After $6 \mathrm{~h}$ at $\mathrm{RT}$, water was added $(20 \mathrm{~mL})$ and the product extracted with $\mathrm{CH}_{2} \mathrm{Cl}_{2}(20 \mathrm{~mL})$ and the combined organic layers were washed with brine and dried over $\mathrm{Na}_{2} \mathrm{SO}_{4}$ and concentrated in vacuo. The crude product was purified by chromatography on silica gel (elution with hexane/Et $\left.\mathrm{Et}_{2} \mathrm{O}: 85 / 15\right)$ to afford $135 \mathrm{mg}(77 \%)$ of the product $\mathbf{7 0}$ as a yellow oil.

$\mathbf{R f}=0.46\left(\right.$ hexane/Et $\left.\mathrm{E}_{2} \mathrm{O}: 50 / 50\right) . \mathbf{N M R}{ }^{1} \mathbf{H}\left(300 \mathrm{MHz}, \mathrm{CDCl}_{3}\right) \delta 6.33(\mathrm{t}, 1 \mathrm{H}, J=3.8 \mathrm{~Hz}), 4.72$ $(\mathrm{s}, 1 \mathrm{H}), 4.30(\mathrm{~s}, 4 \mathrm{H}), 4.27(\mathrm{~s}, 2 \mathrm{H}), 4.21(\mathrm{~d}, 2 \mathrm{H}, J=2.5 \mathrm{~Hz}), 2.44(\mathrm{t}, 1 \mathrm{H}, J=2.5 \mathrm{~Hz})$, 2.21-1.92 (m, 4H), 1.88-1.59 (m, 2H), $1.65(\mathrm{~s}, 3 \mathrm{H}), 1.37(\mathrm{~s}, 3 \mathrm{H}) . \mathbf{N M R}{ }^{13} \mathbf{C}\left(50 \mathrm{MHz}, \mathrm{CDCl}_{3}\right)$ $\delta 134.9,124.2,110.3,84.2,83.4,82.2,81.8,81.2,78.7,75.4,75.0,56.5,56.4,56.3,38.1$, 27.3, 26.2 , 26.1, 19.5. IR (FTIR, film) $: v=3287,2983,2938,2856,2362,2250,2116,158$ è, $1444,1379,1255,1210,1119,1073,1038,989,905,732 \mathrm{~cm}^{-1}$.

(3-\{[4-(\{3-[(4S*,5S*)-6-bromo-2,2-dimethyl-1,3-dioxaspiro[4.5]dec-6-en-4-yl]prop-2ynyl $\}$ oxy)but-2-ynyl]oxy\}prop-1-ynyl)(trimethyl)silane (5)

Compound 5 was prepared following the same procedure than for the preparation of $\mathbf{1}$, starting from compound 70 (130 mg, $0.32 \mathrm{mmol}, 1$ eq.), $n$-BuLi ( $0.3 \mathrm{~mL}, 0.34 \mathrm{mmol}, 1.05$ eq.) and $\mathrm{TMSCl}$ (38 mg, $0.35 \mathrm{mmol}, 1.09$ eq.) in anhydrous THF (7 mL). After an aqueous treatment and chromatography on silica gel (elution with hexane/Et $\left.{ }_{2} \mathrm{O}: 85 / 15\right), 120 \mathrm{mg}(78 \%$ ) of product 5 was obtained as a colorless oil.

$\mathbf{R f}=0.70\left(\right.$ hexane/Et $\left.{ }_{2} \mathrm{O}: 50 / 50\right) . \mathbf{N M R}^{1} \mathbf{H}\left(300 \mathrm{MHz}, \mathrm{CDCl}_{3}\right) \delta 6.36(\mathrm{t}, 1 \mathrm{H}, J=4.1 \mathrm{~Hz}), 4.75$ $(\mathrm{s}, 1 \mathrm{H}), 4.40-4.25(\mathrm{~m}, 6 \mathrm{H}), 4.23(\mathrm{~s}, 2 \mathrm{H}), 2.24-1.94(\mathrm{~m}, 4 \mathrm{H}), 1,90-1.59(\mathrm{~m}, 2 \mathrm{H}), 1.68(\mathrm{~s}, 3 \mathrm{H})$, $1.39(\mathrm{~s}, 3 \mathrm{H}), 0.17(\mathrm{~s}, 9 \mathrm{H}) . \mathbf{N M R}{ }^{13} \mathbf{C}\left(50 \mathrm{MHz}, \mathrm{CDCl}_{3}\right) \delta 135.0,124.3,110.5,100.4,92.1$, 84.3, 83.5, 82.1, 81.3, 77.6, 75.5, 57.3, 56.6, 56.5, 38.2, 27.4, 26.3, 26,2, 19.6, -0.3. IR (FTIR, film): $v=2944,2856,2361,2175,1443,1377,1345,1251,1210,1162,1120,1075$, $1039,898,845,760,670 \mathrm{~cm}^{-1}$.

$\left(3-\left\{\left[\left(3 a R^{*}, 10 c S^{*}\right)-2,2-d i m e t h y l-5,6,10,10 c-t e t r a h y d r o-4 H, 8 H-f u r o\left[3 ", 4 ": 2^{\prime}, 3^{\prime}\right]\right.\right.\right.$ naphtho[ $\left[8^{\prime}, 1^{\prime}: 1,4,3\right]$ cyclobuta[1,2-d] [1,3] dioxol-7-yl]methoxy\}prop-1-ynyl)(trimethyl) silane (13)

To a solution of compound 5 (110 mg, $0.229 \mathrm{mmol}, 1$ eq.) in anhydrous benzene (10 mL) was added tributylstannylethylene ( $95 \mathrm{mg}, 0.298 \mathrm{mmol}, 5$ eq.) and $\mathrm{Pd}\left(\mathrm{PPh}_{3}\right)_{4}(27 \mathrm{mg}, 0.030 \mathrm{mmol}$, 0.1 eq.). After $5 \mathrm{~h}$ at $90^{\circ} \mathrm{C}$, the crude mixture was evaporated in vacuo and purified by chromatography on silica gel (elution with hexane/Et ${ }_{2} \mathrm{O}: 80 / 20$ ), affording $9 \mathrm{mg}(11 \%)$ of impure compound $\mathbf{1 3}$ as yellow oil.

$\mathbf{R f}=0.55$ (hexane $\left./ \mathrm{Et}_{2} \mathrm{O}: 50 / 50\right) . \mathbf{N M R}{ }^{1} \mathbf{H}\left(300 \mathrm{MHz}, \mathrm{CDCl}_{3}\right) \delta 5.25(\mathrm{~s}, 1 \mathrm{H}), 5.20-5.05(\mathrm{~m}$, $3 \mathrm{H}), 4.94(\mathrm{~m}, 1 \mathrm{H}), 4.53(\mathrm{~d}, 1 \mathrm{H}, J=11.2 \mathrm{~Hz}), 4.44(\mathrm{~d}, 1 \mathrm{H}, J=11.2 \mathrm{~Hz}), 4.16(\mathrm{~s}, 2 \mathrm{H})$, 3.10-2.94 (m, 1H), 2.60-2.42 (m, 1H), 2.38-1.98 (m, 4H), $1.56(\mathrm{~s}, 3 \mathrm{H}), 1.40(\mathrm{~s}, 3 \mathrm{H}), 0.21(\mathrm{~s}$, 9H). NMR ${ }^{13} \mathbf{C}\left(75 \mathrm{MHz}, \mathrm{CDCl}_{3}\right) \delta 148.4,143.9,139.9,133.1,133.1,130.3,114.7,101.2$, $92.0,88.4,83.9,72.5,71.3,65.8,58.1,29.7,29.0,23.9,22.1,-0.2$.

\section{Synthesis of 7 from allylamine and 2-butyn-1,4-diol}




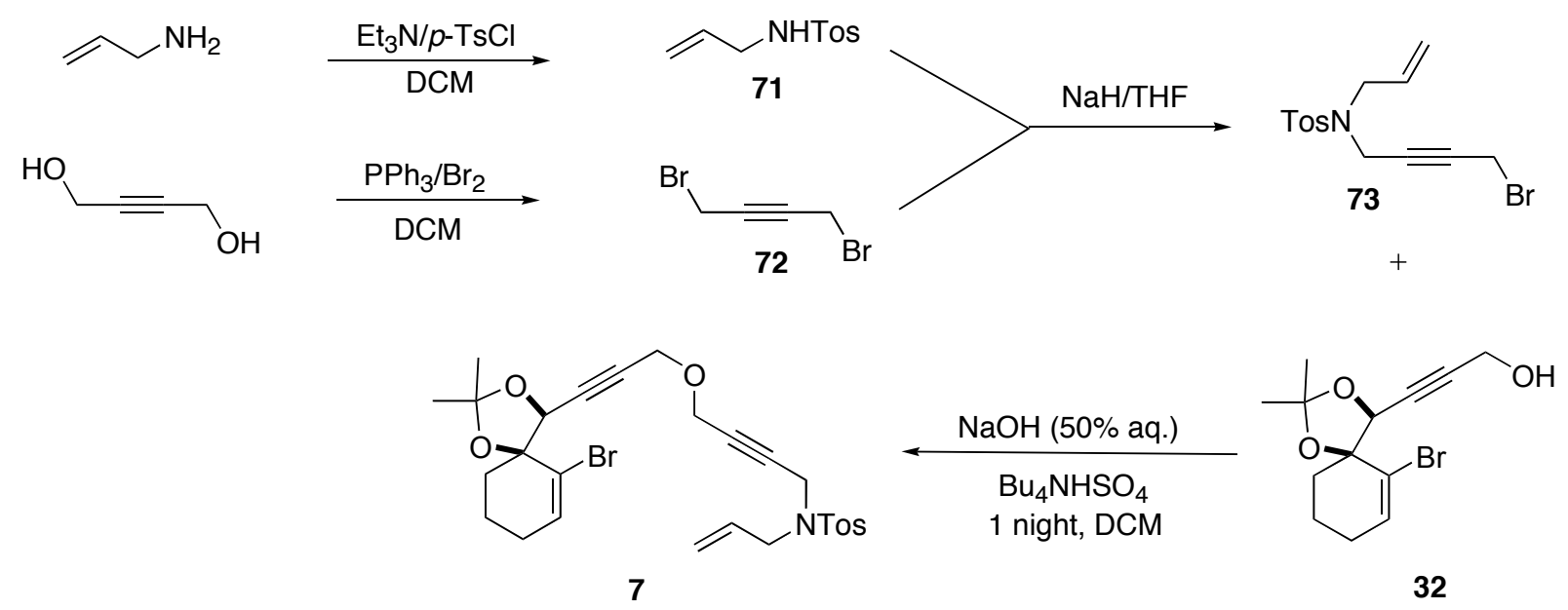

\section{N-allyl-N-(p-tosyl)amine (71)}

To a solution of allylamine ( $2 \mathrm{~g}, 35.0 \mathrm{mmol}, 1.1 \mathrm{eq}$.$) and triethylamine (4.5 \mathrm{~mL}, 31.8 \mathrm{mmol}, 1$ eq.) in $\mathrm{CH}_{2} \mathrm{Cl}_{2}(150 \mathrm{~mL})$ in reflux, was added slowly $p$ - $\mathrm{TsCl}(6 \mathrm{~g}, 31.8 \mathrm{mmol}, 1$ eq. $)$. After $1 \mathrm{~h}$ in reflux and $30 \mathrm{~min}$ at room temperature, the crude reaction was added to a saturated aqueous solution of $\mathrm{NaHCO}_{3}(90 \mathrm{~mL})$. The product was extracted with $\mathrm{CH}_{2} \mathrm{Cl}_{2}(100 \mathrm{~mL})$, washed with brine $(2 * 1 \mathrm{~mL})$ and dried over $\mathrm{Na}_{2} \mathrm{SO}_{4}$, filtrated and evaporated in vacuo. $6.7 \mathrm{~g}(99 \%)$ of compound 71 was obtained as lightly pink solid without any other purification.

$\mathbf{R f}=0.20$ (hexane/Et $\left.{ }_{2} \mathrm{O}: 50 / 50\right) . \mathbf{N M R}^{1} \mathbf{H}\left(300 \mathrm{MHz}, \mathrm{CDCl}_{3}\right) \delta 7.76(\mathrm{~d}, 2 \mathrm{H}, J=7.8 \mathrm{~Hz})$, $7.32(\mathrm{~d}, 2 \mathrm{H}, J=7.8 \mathrm{~Hz}), 5.72(\mathrm{~m}, 1 \mathrm{H}), 5.17(\mathrm{dd}, 1 \mathrm{H}, J=1.5 \mathrm{~Hz}, J=17.1 \mathrm{~Hz}), 5.10(\mathrm{dd}, 1 \mathrm{H}, J$ $=1.5 \mathrm{~Hz}, J=10.5 \mathrm{~Hz}), 4.59(\mathrm{~s}, 1 \mathrm{H}), 3.58(\mathrm{~m}, 2 \mathrm{H}), 2.44(\mathrm{~s}, 3 \mathrm{H}) . \mathbf{N M R}{ }^{13} \mathbf{C}\left(50 \mathrm{MHz}, \mathrm{CDCl}_{3}\right)$ $\delta 143.2,136.6,132.7,129.4,126.9,117.2,45.4,21.2$.

\section{1,4-dibromobut-2-yne (72)}

To a solution of triphenylphosphine $\left(25.2 \mathrm{~g}, 86.0 \mathrm{mmol}, 1.7 \mathrm{eq}\right.$.) in anhydrous $\mathrm{CH}_{2} \mathrm{Cl}_{2}(300$ $\mathrm{mL})$ at $0^{\circ} \mathrm{C}$ was added slowly $\mathrm{Br}_{2}(5 \mathrm{~mL}, 97.0 \mathrm{mmol}, 2.1$ eq. $)$. After $1 \mathrm{~h}$ at $0^{\circ} \mathrm{C}$, the crude mixture was cooled down to $-6^{\circ} \mathrm{C}$ and 2-butyn-1,4-diol (3.9 g, $45.3 \mathrm{mmol}, 1$ eq.) was added. After $2 \mathrm{~h}$ at this temperature, a saturated aqueous solution of $\mathrm{NaHCO}_{3}$ was added and the combined extracts were washed with brine and dried over $\mathrm{Na}_{2} \mathrm{SO}_{4}$. Triphenylphosphine oxide was removed by precipitation in hexane and filtration. A chromatography on silica gel (elution with hexane/ $\left.\mathrm{Et}_{2} \mathrm{O}: 80 / 20\right)$ afford $4.6 \mathrm{~g}(48 \%$ ) of the product 72 as a yellow liquid.

$\mathbf{R f}=0.68$ (hexane/Et $2 \mathrm{O}: 80 / 20) . \mathbf{N M R}^{\mathbf{1}} \mathbf{H}\left(200 \mathrm{MHz}, \mathrm{CDCl}_{3}\right) \delta 3.96(\mathrm{~s}, 4 \mathrm{H}) . \mathbf{N M R}{ }^{\mathbf{1 3}} \mathbf{C}(75$ $\left.\mathrm{MHz}, \mathrm{CDCl}_{3}\right) \delta 81.5,14.0$.

\section{$N$-allyl- $N$-(p-tosyl)-l-amino-4-bromobut-2-yne (73)}

To a suspension of $\mathrm{NaH}(108 \mathrm{mg}, 2.7 \mathrm{mmol}, 1.1$ eq. $)$ in anhydrous $\mathrm{THF}(25 \mathrm{~mL})$ at $0^{\circ} \mathrm{C}$, was added compound 71 (500 mg, $2.4 \mathrm{mmol}, 1$ eq.). After $2 \mathrm{~h}$ at $0^{\circ} \mathrm{C}$, was added the $72(1 \mathrm{~g}, 4.7$ mmol, 2 eq.). After $16 \mathrm{~h}$ at room temperature, the reaction mixture was evaporated and solubilised in $\mathrm{CH}_{2} \mathrm{Cl}_{2}$. The organic phase was washed with water, then brine, dried over $\mathrm{Na}_{2} \mathrm{SO}_{4}$, filtrated and concentrated in vacuo. A chromatography on silica gel (elution with hexane/ $\left.\mathrm{CH}_{2} \mathrm{Cl}_{2}: 50 / 50\right)$ afford $582 \mathrm{mg}(63 \%)$ of the product 73 as an orange liquide.

$\mathbf{R f}=0.46\left(\mathrm{Et}_{2} \mathrm{O} /\right.$ hexane: $\left.50 / 50\right) . \mathbf{N M R}^{1} \mathbf{H}\left(200 \mathrm{MHz}, \mathrm{CDCl}_{3}\right) \delta 7.71(\mathrm{~d}, 2 \mathrm{H}, J=6.6 \mathrm{~Hz}), 7.31$ $(\mathrm{d}, 2 \mathrm{H}, J=6.6 \mathrm{~Hz}), 5.85-5.16(\mathrm{~m}, 1 \mathrm{H}), 5.40-5.17(\mathrm{~m}, 2 \mathrm{H}), 4.11(\mathrm{br} \mathrm{s}, 2 \mathrm{H}), 3.77(\mathrm{~d}, 2 \mathrm{H}, J=$ 
$6.4 \mathrm{~Hz}), 3.60(\mathrm{t}, 2 \mathrm{H}, J=2.2 \mathrm{~Hz}), 2.41(\mathrm{~s}, 3 \mathrm{H}) . \mathbf{N M R}{ }^{13} \mathbf{C}\left(50 \mathrm{MHz}, \mathrm{CDCl}_{3}\right) \delta 143.6,135.6$, $131.7,129.5,127.6,127.6,120.0,80.5,79.5,49.1,37.6,36.0,21.5,13.6$.

N-allyl-N-[4-(\{3-[(4S*,5S*)-6-bromo-2,2-dimethyl-1,3-dioxaspiro[4.5]dec-6-en-4-yl]prop2-ynyl\}oxy)but-2-ynyl]-4-methylbenzenesulfonamide (7)

Compound 7 was prepared following the same procedure than for the preparation of $\mathbf{6 1}$, starting from compound 32 (200 mg, $0.664 \mathrm{mmol}, 1$ eq.), compound 73 (490 mg, 1.432 mmol, 2,2 eq.), $n \mathrm{Bu}_{4} \mathrm{NHSO}_{4}(23 \mathrm{mg}, 0.066 \mathrm{mmol}, 0.1$ eq.) and $1 \mathrm{~mL}$ of solution of $\mathrm{NaOH}$ (50\% aq.) in $\mathrm{CH}_{2} \mathrm{Cl}_{2}(3 \mathrm{~mL})$. Aqueous treatment and chromatography on silica gel (elution with hexane/AcOEt: 80/20) afford $319 \mathrm{mg}(85 \%)$ of the product 7 as a yellow oil.

$\mathbf{R f}=0.36\left(\right.$ hexane/Et $\left.{ }_{2} \mathrm{O}: 50 / 50\right) . \mathbf{N M R}^{1} \mathbf{H}\left(300 \mathrm{MHz}, \mathrm{CDCl}_{3}\right) \delta 7.69(\mathrm{~d}, 2 \mathrm{H}, J=7.9 \mathrm{~Hz}), 7.26$ $(\mathrm{d}, 2 \mathrm{H}, J=7.9 \mathrm{~Hz}), 6.26(\mathrm{t}, 1 \mathrm{H}, J=4.2 \mathrm{~Hz}), 5.80-5.95(\mathrm{~m}, 1 \mathrm{H}), 5.23(\mathrm{~d}, 1 \mathrm{H}, J=18.8 \mathrm{~Hz})$, $5.18(\mathrm{~d}, 1 \mathrm{H}, J=10.9 \mathrm{~Hz}), 4.69(\mathrm{~s}, 1 \mathrm{H}), 4.09-3.98(\mathrm{~m}, 6 \mathrm{H}), 3.82-3.75(\mathrm{~m}, 2 \mathrm{H}), 2.38(\mathrm{~s}, 3 \mathrm{H})$, 2.15-1.87 (m, 4H), 1.80-1.55 (m, 2H), $1.62(\mathrm{~s}, 3 \mathrm{H}), 1.35(\mathrm{~s}, 3 \mathrm{H}) . \mathbf{N M R}{ }^{13} \mathbf{C}\left(75 \mathrm{MHz}, \mathrm{CDCl}_{3}\right)$ $\delta 143.3,135.8,134.7,131.7,129.3,127.4,124.2,119.6,110.2,83.9,83.3,81.1,80.6,79.2$, $75.2,56.2,56.0,48.9,37.9,35.8,27.2,26.1$ et $26.0,21.3,19.4$.

\section{Synthesis of 22 from 32}

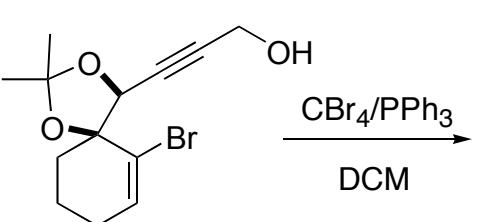

32

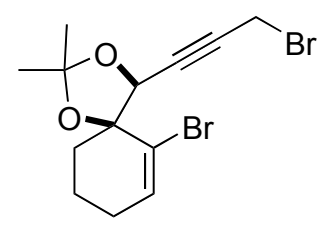

34

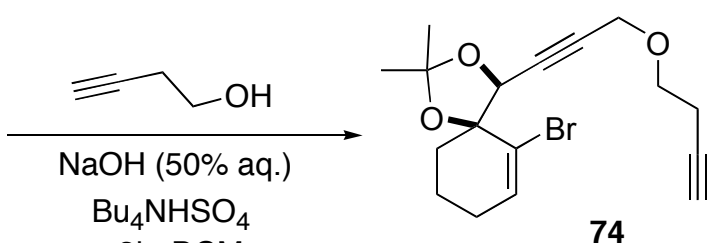

3h, DCM

74

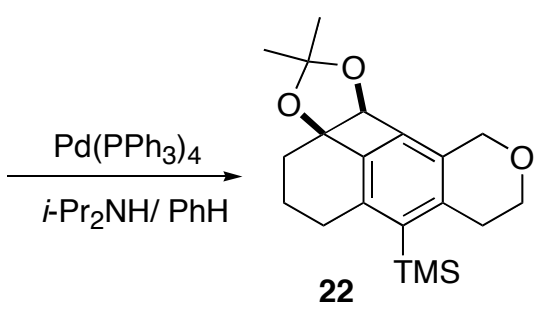

38

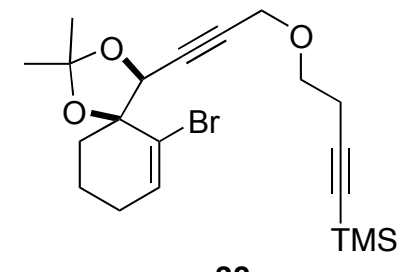

22

$\left(4 S^{*}, 5 S^{*}\right)$-6-bromo-4-(3-bromoprop-1-ynyl)-2,2-dimethyl-1,3-dioxaspiro[4.5]dec-6-ene (34)

To a solution of compound $32\left(414 \mathrm{mg}, 1.37 \mathrm{mmol}, 1\right.$ eq.) in $\mathrm{CH}_{2} \mathrm{Cl}_{2}(8 \mathrm{~mL})$ at $0^{\circ} \mathrm{C}$ was added $\mathrm{PPh}_{3}$ (433 mg, $1.65 \mathrm{mmol}, 1.2$ eq.) then $\mathrm{CBr}_{4}(547 \mathrm{mg}, 1.65 \mathrm{mmol}, 1.2$ eq.). After $30 \mathrm{~min}$ at $0^{\circ} \mathrm{C}$ and $2 \mathrm{~h}$ at room temperature, The reaction mixture was evaporated in vacuo, and chromatographied on silica gel (elution with heptane $\left./ \mathrm{Et}_{2} \mathrm{O}: 90 / 10\right)$ affording $409 \mathrm{mg}(82 \%)$ of the product $\mathbf{3 4}$ as a yellow oil.

$\mathbf{R f}=0.33($ heptane/Et $2 \mathrm{O}: 85 / 15) . \mathbf{N M R}{ }^{1} \mathbf{H}\left(200 \mathrm{MHz}, \mathrm{CDCl}_{3}\right) \delta 6.38(\mathrm{t}, 1 \mathrm{H}, J=4.2 \mathrm{~Hz})$, $4.77(\mathrm{t}, 1 \mathrm{H}, J=1.8 \mathrm{~Hz}), 3.97(\mathrm{~d}, 2 \mathrm{H}, J=1.8 \mathrm{~Hz}), 2.17-2.01(\mathrm{~m}, 4 \mathrm{H}), 1.87-1.75(\mathrm{~m}, 2 \mathrm{H})$, $1.70(\mathrm{~s}, 3 \mathrm{H}), 1.41(\mathrm{~s}, 3 \mathrm{H}) . \mathbf{N M R}{ }^{13} \mathbf{C}\left(50 \mathrm{MHz}, \mathrm{CDCl}_{3}\right) \delta 135.0,123.9,110.6,83.8,83.7,81.1$, 75.3, 38.1, 27.4, 26.3, 19.6, 14.0. MS (apparatus 6) : 385.0, 387.0, $388.9\left[\mathrm{M}+\mathrm{Na}^{+}\right]$. 
Compound 74 was prepared following the same procedure than for the preparation of $\mathbf{6 1}$, starting from compound 34 (100 mg, $0.275 \mathrm{mmol}, 1$ eq.), but-1-ol-3-yne ( $35 \mu \mathrm{L}, 0.413 \mathrm{mmol}$, 1.5 eq.), $n \mathrm{Bu}_{4} \mathrm{NHSO}_{4}(9 \mathrm{mg}, 0.027 \mathrm{mmol}, 0.1$ eq.) and $0.5 \mathrm{~mL}$ of solution of $\mathrm{NaOH}(50 \%$ aq.) in $\mathrm{CH}_{2} \mathrm{Cl}_{2}(2 \mathrm{~mL})$. Aqueous treatment and chromatography on silica gel (elution with heptane $\left./ \mathrm{Et}_{2} \mathrm{O}: 85 / 15\right)$ afford $86 \mathrm{mg}(89 \%)$ of the product 74 as a yellow oil.

$\mathbf{R f}=0.22$ (heptane/Et $2 \mathrm{O}: 85 / 15) . \mathbf{N M R}^{1} \mathbf{H}\left(300 \mathrm{MHz}, \mathrm{CDCl}_{3}\right) \delta 6.36(\mathrm{t}, 1 \mathrm{H}, J=4.1 \mathrm{~Hz})$, $4.74($ br s, $1 \mathrm{H}), 4.27$ (d, 2H, $J=1.6 \mathrm{~Hz}), 3.72-3.64(\mathrm{~m}, 2 \mathrm{H}), 2.48$ (dt, $2 \mathrm{H}, J=2.7 \mathrm{~Hz}, J=7.0$ $\mathrm{Hz}), 2.14-1.98(\mathrm{~m}, 5 \mathrm{H}), 1.82-1.71(\mathrm{~m}, 2 \mathrm{H}), 1.68(\mathrm{~s}, 3 \mathrm{H}), 1.39(\mathrm{~s}, 3 \mathrm{H}) . \mathbf{N M R}^{13} \mathbf{C}(75 \mathrm{MHz}$, $\left.\mathrm{CDCl}_{3}\right) \delta 134.9,124.3,110.4,84.8,83.5,80.9,80.8,75.5,69.3,67.4,58.2,38.2,27.4,26.3$, 26.2, 19.6. ${ }^{3} \mathrm{MS}$ (Apparatus 4, ESI, positif ion): 375.0, $377.0\left[\mathrm{M}+\mathrm{Na}^{+}\right], 391.0,393.0\left[\mathrm{M}+\mathrm{K}^{+}\right]$.

\section{[4-(\{3-[(4S*,5S*)-6-bromo-2,2-dimethyl-1,3-dioxaspiro[4.5]dec-6-en-4-yl]prop-2- ynyl\}oxy)but-1-ynyl](trimethyl)silane (38)}

Compound 38 was prepared following the same procedure than for the preparation of $\mathbf{1}$, starting from compound 74 ( $80 \mathrm{mg}, 0.240 \mathrm{mmol}, 1$ eq.), $n$-BuLi $(0.200 \mathrm{~mL}, 0.260 \mathrm{mmol}, 1.1$ eq.) and $\mathrm{TMSCl}(33 \mu \mathrm{L}, 0.260 \mathrm{mmol}, 1.1$ eq.) in anhydrous THF (3 mL). After an aqueous treatment of the reaction mixture $(10 \mathrm{~mL})$ and chromatography on silica gel (elution with heptane/Et $\left.{ }_{2} \mathrm{O}: 85 / 15\right), 30 \mathrm{mg}(29 \%)$ of product 38 was obtained as a yellow oil.

$\mathbf{R f}=0.26\left(\right.$ hexane $\left./ \mathrm{Et}_{2} \mathrm{O}: 80 / 20\right) . \mathbf{N M R}^{1} \mathbf{H}\left(300 \mathrm{MHz}, \mathrm{CDCl}_{3}\right) \delta 6.38(\mathrm{t}, 1 \mathrm{H}, J=4.2 \mathrm{~Hz}), 4.76$ (t, $1 \mathrm{H}, J=1.7 \mathrm{~Hz}), 4.26(\mathrm{~d}, 2 \mathrm{H}, J=1.7 \mathrm{~Hz}), 3.68(\mathrm{t}, 2 \mathrm{H}, J=7.4 \mathrm{~Hz}), 2.53(\mathrm{t}, 2 \mathrm{H}, J=7.4 \mathrm{~Hz})$, 2.15-1.98 (m, 4H), 1.88-1.73 (m, 2H), $1.70(\mathrm{~s}, 3 \mathrm{H}), 1.41(\mathrm{~s}, 3 \mathrm{H}), 0.15(\mathrm{~s}, 9 \mathrm{H}) . \mathbf{N M R}^{13} \mathbf{C}(75$ $\left.\mathrm{MHz}, \mathrm{CDCl}_{3}\right) \delta 135.0,124.5,110.5,103.2,85.9,85.0,83.6,80.8,75.6,67.7,58.3,38.3,27.5$, 26.32, 26.26, 21.0, 19.7, 0.1. MS (Apparatus 4, ESI, positif ion): 463.0, $465.1\left[\mathrm{M}+\mathrm{K}^{+}\right]$.

$\left[\left(3 \mathrm{a} R^{*}, 11 \mathrm{c} S *\right)-2,2-\right.$ dimethyl-5,6,8,9,11,11c-hexahydro-4H[1,3]dioxolo[1',4']cyclobuta[3',2',1':1,8]naphtho[2,3-c]pyran-7-yl](trimethyl)silane (22)

To a solution of compound 38 (30 mg, $0.07 \mathrm{mmol}, 1$ eq.) in anhydrous benzene $(0.6 \mathrm{~mL})$ was added $\mathrm{Pd}\left(\mathrm{PPh}_{3}\right)_{4}(8 \mathrm{mg}, 0,007 \mathrm{mmol}, 0,1$ eq. $)$ and diisopropyl amine $(0.2 \mathrm{~mL})$. The mixture was purged $5 \mathrm{~min}$ with argon and heated at $130^{\circ} \mathrm{C}$ under microwave irradiation during 20 min. The reaction mixture was concentrated in vacuo and purified by chromatography on silica gel (elution with heptane/Et $\left.\mathrm{Et}_{2} \mathrm{O}: 85 / 15\right)$ to afford $15 \mathrm{mg}(62 \%)$ of 22 as a yellow solid.

$\mathbf{R f}=0.22\left(\right.$ hexane/Et $\left.{ }_{2} \mathrm{O}: 85 / 15\right) . \mathbf{M p}=102^{\circ} \mathrm{C} . \mathbf{N M R}{ }^{1} \mathbf{H}\left(300 \mathrm{MHz}, \mathrm{CDCl}_{3}\right) \delta 5.18(\mathrm{~s}, 1 \mathrm{H})$, $4.89(\mathrm{~d}, 1 \mathrm{H}, J=15.4 \mathrm{~Hz}), 4.72(\mathrm{~d}, 1 \mathrm{H}, J=15.4 \mathrm{~Hz}), 3.95(\mathrm{t}, 2 \mathrm{H}, J=5.6 \mathrm{~Hz}), 3.12(\mathrm{dd}, 1 \mathrm{H}, J$ $=17.1 \mathrm{~Hz}, J=6.3 \mathrm{~Hz}), 2.99-2.94(\mathrm{~m}, 2 \mathrm{H}), 2.47-2.00(\mathrm{~m}, 4 \mathrm{H}), 1.61-1.56(\mathrm{~m}, 1 \mathrm{H}), 1.50(\mathrm{~s}$, $3 \mathrm{H}), 1.40(\mathrm{~s}, 3 \mathrm{H}), 0.37(\mathrm{~s}, 9 \mathrm{H}) . \mathbf{N M R}{ }^{13} \mathbf{C}\left(75 \mathrm{MHz}, \mathrm{CDCl}_{3}\right) \delta 145.2,143.2,142.9,139.7$, $138.8,128.3,114.5,88.3,82.8,65.8,65.5,31.7,28.9,28.8,28.7,28.2,22.7,2.8$. GC-MS (Apparatus 1): $344.0\left[\mathrm{M}^{+\bullet}\right], 286,213$, 73. HRMS (ESI, positive ion): calc'd for $\left(\mathrm{C}_{20} \mathrm{H}_{28} \mathrm{O}_{3} \mathrm{NaSi}\right)^{+} 367.1711$; found 367.1700 . COSY, HSQC, HMBC, NOESY

\section{Synthesis of 23 from 34}

\footnotetext{
${ }^{3}$ In this spectra one carbone is not visible, $\mathrm{CH}$ alkyne.
} 


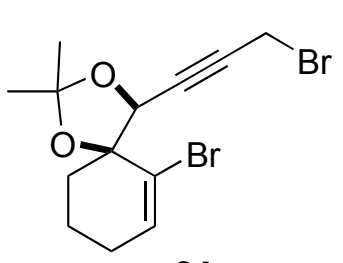

34

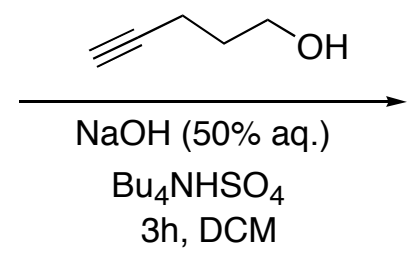

3h, DCM

1) $n$-BuLi/THF

2) TMSCl

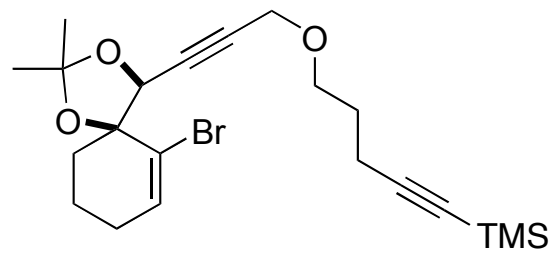

39

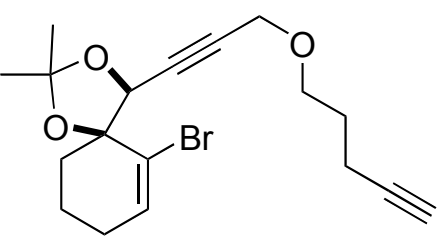

75

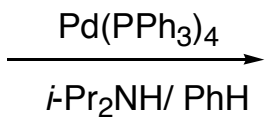

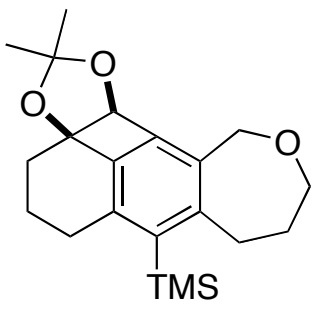

23

$\left(4 S^{*}, 5 S^{*}\right)-6$-bromo-2,2-dimethyl-4-[3-(pent-4-ynyloxy)prop-1-ynyl]-1,3-dioxaspiro [4.5]dec-6-ene (75)

Compound 75 was prepared following the same procedure than for the preparation of $\mathbf{6 1}$, starting from compound $34(80 \mathrm{mg}, 0.220 \mathrm{mmol}, 1 \mathrm{eq}$.), pent-1-ol-4-yne (30 $\mu \mathrm{L}, 0.330 \mathrm{mmol}$, 1.5 eq.), $n \mathrm{Bu}_{4} \mathrm{NHSO}_{4}$ ( $8 \mathrm{mg}, 0.022 \mathrm{mmol}, 0.1$ eq.) and $0.4 \mathrm{~mL}$ of solution of $\mathrm{NaOH}(50 \%$ aq.) in $\mathrm{CH}_{2} \mathrm{Cl}_{2}(1.6 \mathrm{~mL})$. Aqueous treatment $(10 \mathrm{~mL}$ of brine) and chromatography on silica gel (elution with heptane/ $\left.\mathrm{Et}_{2} \mathrm{O}: 85 / 15\right)$ afford $67 \mathrm{mg}$ (83\%) of the product 75 as a yellow oil.

$\mathbf{R f}=0.23$ (heptane/Et $2 \mathrm{O}: 85 / 15) . \mathbf{N M R}^{1} \mathbf{H}\left(300 \mathrm{MHz}, \mathrm{CDCl}_{3}\right) \delta 6.34(\mathrm{t}, 1 \mathrm{H}, J=4.1 \mathrm{~Hz})$, $4.74(\mathrm{br} \mathrm{s}, 1 \mathrm{H}), 4.21(\mathrm{~d}, 2 \mathrm{H}, J=1.6 \mathrm{~Hz}), 3.62(\mathrm{dt}, 2 \mathrm{H}, J=2.2 \mathrm{~Hz}, J=6.2 \mathrm{~Hz}), 2.48(\mathrm{dt}, 2 \mathrm{H}$, $J=2.7 \mathrm{~Hz}, J=7.2 \mathrm{~Hz}), 2.13-1.97(\mathrm{~m}, 4 \mathrm{H}), 1.93(\mathrm{t}, 1 \mathrm{H}, J=2.7 \mathrm{~Hz}), 1.84-1.73(\mathrm{~m}, 2 \mathrm{H}), 1.8(\mathrm{t}$, $1 \mathrm{H}, J=6.6 \mathrm{~Hz}), 1.68(\mathrm{~s}, 3 \mathrm{H}), 1.39(\mathrm{~s}, 3 \mathrm{H}) . \mathbf{N M R}^{13} \mathbf{C}\left(75 \mathrm{MHz}, \mathrm{CDCl}_{3}\right) \delta 134.8,124.4,110.4$, $85.3,83.8,83.5,80.4,75.6,68.4,68.2,58.3,38.2,28.5,27.5,26.3,26.2,19.6,15.2$.

\section{[5-(\{3-[(4S*,5S*)-6-bromo-2,2-dimethyl-1,3-dioxaspiro[4.5] dec-6-en-4-yl]prop-2- ynyl\}oxy)pent-1-ynyl](trimethyl)silane (39)}

Compound 39 was prepared following the same procedure than for the preparation of $\mathbf{1}$, starting from compound 75 (67 mg, $0.182 \mathrm{mmol}, 1$ eq.), $n$-BuLi (0.130 mL, $0.201 \mathrm{mmol}, 1.1$ eq.) and $\mathrm{TMSCl}(26 \mu \mathrm{L}, 0.201 \mathrm{mmol}, 1.1$ eq.) in anhydrous THF $(2 \mathrm{~mL})$. After an aqueous treatment and chromatography on silica gel (elution with heptane/Et $\left.\mathrm{t}_{2} \mathrm{O}: 85 / 15\right), 22 \mathrm{mg}(28 \%)$ of product 39 was obtained as a yellow oil.

$\mathbf{R f}=0.27$ (heptane/Et $\left.{ }_{2} \mathrm{O}: 80 / 20\right) . \mathbf{N M R}{ }^{1} \mathbf{H}\left(200 \mathrm{MHz}, \mathrm{CDCl}_{3}\right) \delta 6.36(\mathrm{t}, 1 \mathrm{H}, J=4.2 \mathrm{~Hz}), 4.76$ $(\mathrm{t}, 1 \mathrm{H}, J=1.6 \mathrm{~Hz}), 4.23(\mathrm{~d}, 2 \mathrm{H}, J=1.6 \mathrm{~Hz}), 3.62(\mathrm{t}, 2 \mathrm{H}, J=6.7 \mathrm{~Hz}), 2.32(\mathrm{t}, 2 \mathrm{H}, J=6.7 \mathrm{~Hz})$, 2.16-1.93 (m, 4H), 1.86-1.73 (m, 4H), $1.69(\mathrm{~s}, 3 \mathrm{H}), 1.41(\mathrm{~s}, 3 \mathrm{H}), 0.15(\mathrm{~s}, 9 \mathrm{H}) . \mathbf{N M R}{ }^{13} \mathbf{C}(75$ $\left.\mathrm{MHz}, \mathrm{CDCl}_{3}\right) \delta 134.8,124.5,110.5,106.7,85.5,84.7,83.6,80.5,75.6,68.5,58.3,38.3,28.7$, $27.5,26.4,26.32,19.7,16.7,0.2$.

\section{$\left[\left(3 \mathrm{a} R^{*}, 12 \mathrm{c} S^{*}\right)\right.$-2,2-dimethyl-5,6,9,10,12,12c-hexahydro-4H,8H-}

$[1,3]$ dioxolo[1',4']cyclobuta[3',2',1':1,8]naphtho[2,3-c]oxepin-7-yl](trimethyl)silane (23)

To a solution of compound $39(21 \mathrm{mg}, 0.05 \mathrm{mmol}, 1$ eq.) in anhydrous benzene $(0.4 \mathrm{~mL})$ was added $\mathrm{Pd}\left(\mathrm{PPh}_{3}\right)_{4}(6 \mathrm{mg}, 0,005 \mathrm{mmol}, 0,1$ eq. $)$ and diisopropyl amine $(0.15 \mathrm{~mL})$. The mixture was purged 5 min with argon and heated at $130^{\circ} \mathrm{C}$ under microwave irradiation during $4 * 10$ min. The reaction mixture was concentrated in vacuo and purified by chromatography on 
silica gel (elution with heptane/ $\left.\mathrm{Et}_{2} \mathrm{O}: 80 / 20\right)$ to afford $13 \mathrm{mg}(72 \%)$ of $\mathbf{2 3}$ as a yellow solid.

$\mathbf{R f}=0.16\left(\right.$ heptane $\left./ \mathrm{Et}_{2} \mathrm{O}: 20 / 80\right) . \mathbf{M p}=92^{\circ} \mathrm{C} . \mathbf{N M R}{ }^{1} \mathbf{H}\left(300 \mathrm{MHz}, \mathrm{CDCl}_{3}\right) \delta 5.30(\mathrm{~s}, 1 \mathrm{H})$, $4.75(\mathrm{~d}, 1 \mathrm{H}, J=13.9 \mathrm{~Hz}), 4.58(\mathrm{~d}, 1 \mathrm{H}, J=13.9 \mathrm{~Hz}), 4.18-4.12(\mathrm{~m}, 1 \mathrm{H}), 3.96-3.88(\mathrm{~m}, 1 \mathrm{H})$, $3.28-3,02(\mathrm{~m}, 3 \mathrm{H}), 2.41-2.01(\mathrm{~m}, 4 \mathrm{H}), 1.86-1.76(\mathrm{~m}, 2 \mathrm{H}), 1.57(\mathrm{dd}, 1 \mathrm{H}, J=2.7 \mathrm{~Hz}, J=13.1$ $\mathrm{Hz}), 1.50(\mathrm{~s}, 3 \mathrm{H}), 1.39(\mathrm{~s}, 3 \mathrm{H}), 0.37(\mathrm{~s}, 9 \mathrm{H}) . \mathbf{N M R}{ }^{13} \mathbf{C}\left(50 \mathrm{MHz}, \mathrm{CDCl}_{3}\right) \delta 153.0,146.4$, $144.7,140.5,138.4,132.9,114.4,87.3,82.8,75.2,69.2,34.5,30.6,29.1,28.8,28.6,28.3$, 22.8, 3.3. HRMS (apparatus 2, ESI, positive ion): calc'd for $\left(\mathrm{C}_{21} \mathrm{H}_{30} \mathrm{O}_{3} \mathrm{NaSi}\right)^{+} 381.1865$; found 381.1856 .

\section{Synthesis of 24 from 34}<smiles>CC1(C)OC(C#CCBr)C2(CCCC=C2Br)O1</smiles>

34

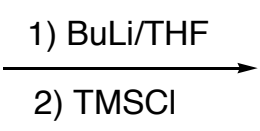

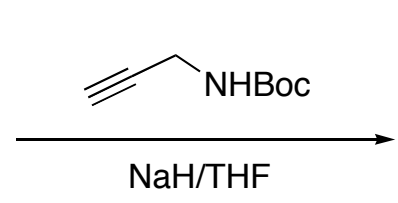<smiles>CC(C)(C)OC(=O)CC#CCC#CC1OC(C)(C)OC12CCCC=C2Br</smiles>

40<smiles>C#CCN(CC#CC1OC(C)(C)OC12CCCC=C2Br)C(=O)O</smiles>

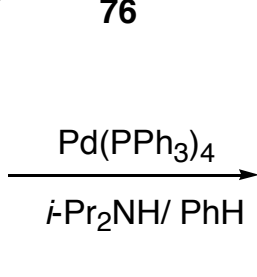

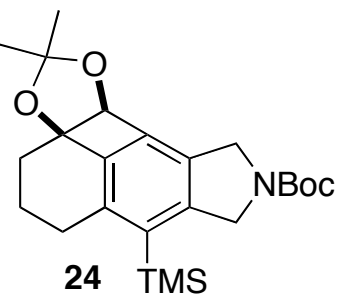

tert-butyl-3-[(4S*,5S*)-6-bromo-2,2-dimethyl-1,3-dioxaspiro[4.5]dec-6-en-4-yl]prop-2ynyl(prop-2-ynyl)carbamate (76)

To a suspension of $\mathrm{NaH}(107 \mathrm{mg}, 0.294 \mathrm{mmol}, 1$ eq., $60 \%$ dispersion in mineral oil) in anhydrous THF $(1 \mathrm{~mL})$ at $0^{\circ} \mathrm{C}$ under argon atmosphere was added $N$-(tertbutoxycarbonyl)propargylamine ${ }^{4}\left(46 \mathrm{mg}, 0.294 \mathrm{mmol}, 1.0\right.$ eq.). After $1 \mathrm{~h}$ at $0^{\circ} \mathrm{C}$, a solution of compound 34 (107 mg, $0.294 \mathrm{mmol}, 1$ eq.) in THF $(1 \mathrm{~mL})$ was added. Aqueous treatment with brine $(10 \mathrm{~mL})$ and chromatography on silica gel (elution with heptane/ $\left.\mathrm{Et}_{2} \mathrm{O}: 95 / 5\right)$ afford $82 \mathrm{mg}(63 \%)$ of the product 76 as a yellow oil.

$\mathbf{R f}=0.25($ heptane/Et $2 \mathrm{O}: 80 / 20) . \mathbf{N M R}^{1} \mathbf{H}\left(300 \mathrm{MHz}, \mathrm{CDCl}_{3}\right) \delta 6.32(\mathrm{t}, 1 \mathrm{H}, J=4.1 \mathrm{~Hz})$, 4.71 (br s, $1 \mathrm{H}), 4.24$ (br s, 2H), 4.19 (br s, $2 \mathrm{H}), 2.19$ (t, $1 \mathrm{H}, J=2.3 \mathrm{~Hz}), 2.14-1.94(\mathrm{~m}, 4 \mathrm{H})$, 1.83-1.64 (m, 2H), $1.67(\mathrm{~s}, 3 \mathrm{H}), 1.46(\mathrm{~s}, 9 \mathrm{H}), 1.38(\mathrm{~s}, 3 \mathrm{H}) . \mathbf{N M R}^{13} \mathbf{C}\left(75 \mathrm{MHz}, \mathrm{CDCl}_{3}\right) \delta$ 154.0, 134.8, 124.4, 110.4, 84.1, 83.5, 80.9, 78.9, 78.1, 75.46, 71.7 (br s), 38.1, 35.1 (br, s), 28.2, 27.4, 26.2, 19.6. ${ }^{5} \mathrm{MS}$ (apparatus 6) : 460.1, 462.1 [M+Na+].

tert-butyl-3-[(4S*,5S*)-6-bromo-2,2-dimethyl-1,3-dioxaspiro[4.5]dec-6-en-4-yl]prop-2ynyl[3-(trimethylsilyl)prop-2-ynyl]carbamate (40)

Compound 40 was prepared following the same procedure than for the preparation of $\mathbf{1}$, starting from compound 76 ( $80 \mathrm{mg}, 0.182 \mathrm{mmol}, 1$ eq. $), n$-BuLi $(0.140 \mathrm{~mL}, 0.219 \mathrm{mmol}, 1.2$ eq.) and TMSCl (28 $\mu \mathrm{L}, 0.219 \mathrm{mmol}, 1.2$ eq.) in anhydrous THF (2 mL). After an aqueous treatment and chromatography on silica gel (elution with heptane/Et $\left.{ }_{2} \mathrm{O}: 80 / 20\right), 36 \mathrm{mg}(39 \%)$ of product 40 was obtained as a yellow oil. (45\% of starting material was recovered at the

\footnotetext{
${ }^{4}$ Synthesis described in J. Org.Chem. 2007, 72(8), 2897-2905.

${ }^{5} \mathrm{In}$ this spectra one carbone is not visible, $\mathrm{CH}$ alkyne.
} 
end of the reaction).

$\mathbf{R f}=0.40$ (heptane/Et $\left.{ }_{2} \mathrm{O}: 50 / 50\right) . \mathbf{N M R}^{1} \mathbf{H}\left(300 \mathrm{MHz}, \mathrm{CDCl}_{3}\right) \delta 6.34(\mathrm{t}, 1 \mathrm{H}, J=4.1 \mathrm{~Hz}), 4.72$ (br s, 1H), 4.24-4.19 (m, 4H), 2.17-1.96 (m, 4H), 1.88-1.64 (m, 2H), 1.69 (s, 3H), 1.47 (s, 9H), $1.40(\mathrm{~s}, 3 \mathrm{H}), 0.16(\mathrm{~s}, 9 \mathrm{H}) . \mathbf{N M R}{ }^{13} \mathbf{C}\left(75 \mathrm{MHz}, \mathrm{CDCl}_{3}\right) \delta 154.2,134.8,124.5,110.4$, 100.8, 84.4, 83.6, 80.8, 75.6, 38.2, 35.2, 28.3, 27.5, 26.3, 19.7, -0.1. MS (apparatus 4) : 532.1, $534.1\left[\mathrm{M}+\mathrm{Na}^{+}\right], 548.1,550.2\left[\mathrm{M}+\mathrm{K}^{+}\right]$.

\section{tert-butyl(3a $R *, 10 \mathrm{c} S *)-2,2-d i m e t h y l-7-(t r i m e t h y l s i l y l)-5,6,10,10 \mathrm{c}-$ tetrahydro-4H- [1,3]dioxolo[1',4']cyclobuta[3',2',1':1,8]naphtho[2,3-c]pyrrole-9(8H)-carboxylate (24)}

To a solution of compound $\mathbf{4 0}(36 \mathrm{mg}, 0.07 \mathrm{mmol}, 1$ eq.) in anhydrous benzene $(0.6 \mathrm{~mL})$ was added $\mathrm{Pd}\left(\mathrm{PPh}_{3}\right)_{4}(8 \mathrm{mg}, 0,007 \mathrm{mmol}, 0,1$ eq. $)$ and diisopropyl amine $(0.2 \mathrm{~mL})$. The mixture was purged 5 min with argon and heated at $130^{\circ} \mathrm{C}$ under microwave irradiation during 20 min. The reaction mixture was concentrated in vacuo and purified by chromatography on silica gel (elution with heptane/Et $2 \mathrm{O}: 80 / 20)$ to afford $15 \mathrm{mg}(50 \%)$ of $\mathbf{2 4}$ as a yellow oil.

$\mathbf{R f}=0.35$ (heptane/Et $\left.{ }_{2} \mathrm{O}: 50 / 50\right) . \mathbf{M p}=92^{\circ} \mathrm{C} . \mathbf{N M R}{ }^{1} \mathbf{H} .\left(300 \mathrm{MHz}, \mathrm{CDCl}_{3} / \mathrm{MeOH}: 5 / 1\right) \delta$ $5.13(\mathrm{~s}, 1 \mathrm{H}), 4.53(\mathrm{~s}, 2 \mathrm{H}), 4.51(\mathrm{~d}, 1 \mathrm{H}, J=15.3 \mathrm{~Hz}), 4.33(\mathrm{~d}, 1 \mathrm{H}, J=15.3 \mathrm{~Hz}), 2.97(\mathrm{dd}, 1 \mathrm{H}, J$ $=17.2 \mathrm{~Hz}, J=5.9 \mathrm{~Hz}), 2.36-1.91(\mathrm{~m}, 4 \mathrm{H}), 1.50-1.41(\mathrm{~m}, 1 \mathrm{H}), 1.38(\mathrm{~s}, 12 \mathrm{H}), 1.26(\mathrm{~s}, 3 \mathrm{H}), 0.21$ $(\mathrm{s}, 9 \mathrm{H}) . \mathbf{N M R}{ }^{13} \mathbf{C}\left(75 \mathrm{MHz}, \mathrm{CDCl}_{3}\right) \delta 154.6,154.5,147.2,146.8,142.0,141.8,140.0,139.9$, 134.9, 134.4, 129.5, 129.3, 114.6, 89.0, 83.6, 79.7, 79.6, 53.9, 53.5, 49.4, 48.9, 28.9, 28.9, 28.5, 28.2, 28.1, 22.6, 1.2. COSY, HMQC, HMBC, NOESY. MS (apparatus 6) : 452.2 $\left[\mathrm{M}+\mathrm{Na}^{+}\right]$. HRMS (apparatus 2): calc'd for $\left(\mathrm{C}_{24} \mathrm{H}_{35} \mathrm{NO}_{4} \mathrm{SiNa}\right)^{+} 452.2201$; found 452.2228 .

\section{Synthesis of 25 from 68}

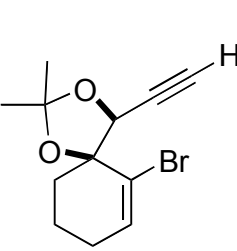

68

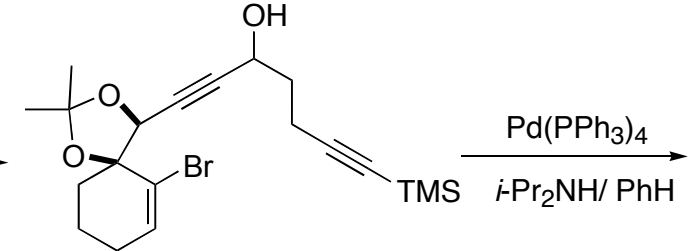

35

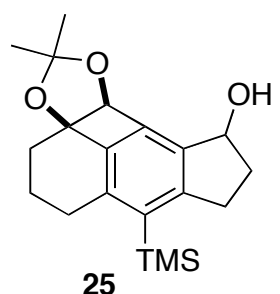

25

$1-\left[\left(4 S^{*}, 5 S^{*}\right)-6\right.$-bromo-2,2-dimethyl-1,3-dioxaspiro[4.5]dec-6-en-4-yl]-7-(trimethylsilyl) hepta-1,6-diyn-3-ol (35)

To a solution of compound 68 (70 $\mathrm{mg}, 0.258 \mathrm{mmol}, 1$ eq.) in anhydrous THF ( $2 \mathrm{~mL})$ under argon atmosphere at room temperature was added $\mathrm{EtMgBr}(0.284 \mathrm{~mL}, 0.284 \mathrm{mmol}, 1.1$ eq. $)$. After $1 \mathrm{~h}$ at $60^{\circ} \mathrm{C}$, a solution of aldehyde $41^{6}(44 \mathrm{mg}, 0.284 \mathrm{mmol}, 1.1$ eq.) in THF ( $1 \mathrm{~mL})$ was added at room temperature. After $2 \mathrm{~h}$ at $40^{\circ} \mathrm{C}$, an aqueous treatment and chromatography on silica gel (elution with pentane/ $\mathrm{Et}_{2} \mathrm{O}$ : $\left.30 / 10\right), 45 \mathrm{mg}$ (41\%) of product 35 was obtained as a yellow oil. ( $46 \%$ of starting material was recovered at the end of the reaction). The two diastereomers were not separated during the chromatography.

$\mathbf{R f}=0.24($ heptane/Et $2 \mathrm{O}: 2 / 1) . \mathbf{N M R}^{1} \mathbf{H}\left(300 \mathrm{MHz}, \mathrm{CDCl}_{3}\right) \delta 6.36(\mathrm{t}, 1 \mathrm{H}, J=4.1 \mathrm{~Hz}), 4.73$ (m, 1H), $4.57(\mathrm{~m}, 1 \mathrm{H}), 2.53-2.25(\mathrm{~m}, 3 \mathrm{H}), 2.14-2.00(\mathrm{~m}, 6 \mathrm{H}), 1.83-1.71(\mathrm{~m}, 2 \mathrm{H}), 1.67(\mathrm{~s}, 3 \mathrm{H})$, 1.39 (s, 3H), 0.14 (s, 9H). NMR ${ }^{13} \mathbf{C}\left(75 \mathrm{MHz}, \mathrm{CDCl}_{3}\right) \delta 134.9,124.4,124.3,110.51,110.47$, 106.1, 106.0, 89.2, 89.1, 85.4, 85.3, 83.7, 79.9, 79.8, 75.4, 75.3, 61.5, 61.4, 38.2, 35.9, 27.4, 
26.3, 19.7, 15.8, 15.7, 0.0. GC-MS (apparatus 1) : 424.1, 426.1 $\left[\mathrm{M}^{+\bullet}\right], 287.1,177.1,159.1$, 73.1.

$\left(3 \mathrm{a} R *, 10 \mathrm{c} S^{*}\right)-2,2-$ dimethyl-7-(trimethylsilyl)-5,6,8,9,10,10c-hexahydro-4Hcyclopenta[2',3']naphtho $\left[8^{\prime}, 1^{\prime}: 1,4,3\right]$ cyclobuta[1,2- $\left.d\right][1,3]$ dioxol-10-ol (25)

To a solution of compound 35 (45 $\mathrm{mg}, 0.106 \mathrm{mmol}, 1$ eq.) in dried benzene $(1.0 \mathrm{~mL})$ was added $\mathrm{Pd}\left(\mathrm{PPh}_{3}\right)_{4}(12 \mathrm{mg}, 0,011 \mathrm{mmol}, 0,1$ eq. $)$ and diisopropyl amine $(0.3 \mathrm{~mL})$. The mixture was purged 5 min with argon and heated at $130^{\circ} \mathrm{C}$ under microwave irradiation during 20 min. The reaction mixture was concentrated in vacuo and purified by chromatography on silica gel (elution with pentane/Et $\left.\mathrm{Et}_{2} \mathrm{O}: 90 / 10\right)$ to afford $11 \mathrm{mg}(30 \%)$ of the one diastereomer (25a) as a yellow solid, $5 \mathrm{mg}(14 \%)$ of a mixture of both diastereomer $\mathbf{2 5 a}$ and $\mathbf{2 5} \mathbf{b}$, and $9 \mathrm{mg}$ of the second diastereomer $25 \mathrm{~b}$ as a yellow oil. The global yield is $68 \%$ and the proportion is $50 / 50$.

25a : Rf $=0.32\left(\right.$ pentane/Et $\left.{ }_{2} \mathrm{O}: 80 / 20\right) . \mathbf{M p}=133^{\circ} \mathrm{C} . \mathbf{~ N M R}^{\mathbf{1}} \mathbf{H}\left(200 \mathrm{MHz}, \mathrm{CDCl}_{3}\right) \delta 5.39-5.32$ $(\mathrm{m}, 2 \mathrm{H}), 3.15-3.01(\mathrm{~m}, 2 \mathrm{H}), 2.91-2.75(\mathrm{~m}, 1 \mathrm{H}), 2.59-1.77(\mathrm{~m}, 7 \mathrm{H}), 1.65-1.56(\mathrm{~m}, 1 \mathrm{H}), 1.50(\mathrm{~s}$, $3 \mathrm{H}), 1.41(\mathrm{~s}, 3 \mathrm{H}), 0.32(\mathrm{~s}, 9 \mathrm{H}) . \mathbf{N M R}{ }^{13} \mathbf{C}\left(50 \mathrm{~Hz}, \mathrm{CDCl}_{3}\right) \delta 153.8,146.3,143.2,140.7,137.3$, 136.1, 114.5, 89.3, 83.1, 73.6, 35.7, 32.9, 28.9, 28.8, 28.4, 28.3, 22.7, 1.6. COSY, HSQC, HMBC, NOESY. GC-MS (apparatus 1) : $344.2\left[\mathrm{M}^{+\bullet}\right], 286.2,269.2,165.1,73.1$.

25b : Rf = 0.24 (pentane/Et $2 \mathrm{O}: 80 / 20)$. NMR $^{1} \mathbf{H}\left(200 \mathrm{MHz}, \mathrm{CDCl}_{3}\right) \delta 5.32(\mathrm{~s}, 1 \mathrm{H}), 5.15(\mathrm{dd}$, $1 \mathrm{H}, J=3.9 \mathrm{~Hz}, J=6.8 \mathrm{~Hz}), 3.24-3.06(\mathrm{~m}, 2 \mathrm{H}), 2.93-2.78(\mathrm{~m}, 1 \mathrm{H}), 2.49-1.82(\mathrm{~m}, 7 \mathrm{H})$, $1.65-1.56(\mathrm{~m}, 1 \mathrm{H}), 1.51(\mathrm{~s}, 3 \mathrm{H}), 1.41(\mathrm{~s}, 3 \mathrm{H}), 0.33(\mathrm{~s}, 9 \mathrm{H}) . \mathbf{N M R}{ }^{13} \mathbf{C}\left(50 \mathrm{~Hz}, \mathrm{CDCl}_{3}\right) \delta 154.2$, 146.3, 143.4, 140.7, 137.9, 136.2, 114.5, 89.0, 83.5, 72.9, 35.0, 32.7, 29.0, 28.9, 28.4, 28.2, 22.7, 1.6. COSY, HSQC, HMBC, NOESY. GC-MS (apparatus 1) : $344.2\left[\mathrm{M}^{+\bullet}\right], 286.2$, 269.2, 165.1, 73.1.

\section{Synthesis of 26 from 68}

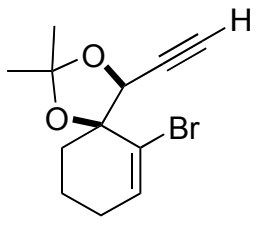

68

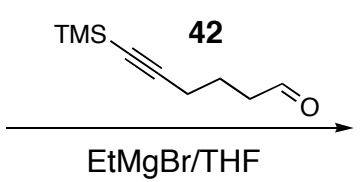

EtMgBr/THF

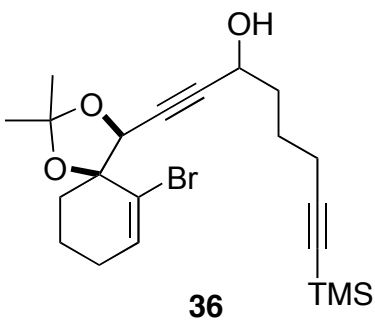

36

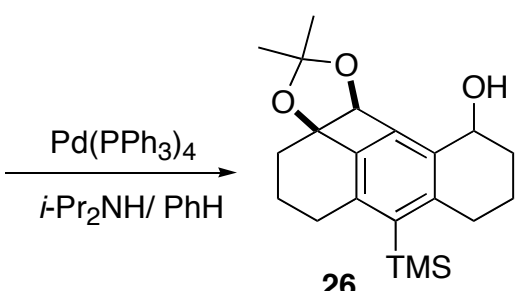

26

\section{1-[(4S*,5 $*)-6$-bromo-2,2-dimethyl-1,3-dioxaspiro[4.5]dec-6-en-4-yl]-8-}

(trimethylsilyl)octa-1,7-diyn-3-ol (36)

To a solution of compound 36 (70 mg, $0.258 \mathrm{mmol}, 1$ eq.) in anhydrous THF ( $2 \mathrm{~mL})$ under argon at room temperature was added $\mathrm{EtMgBr}(0.284 \mathrm{~mL}, 0.284 \mathrm{mmol}, 1.1$ eq. $)$. After $1 \mathrm{~h}$ at $60^{\circ} \mathrm{C}$, a solution of aldehyde $\mathbf{4 2}^{7}$ (48 $\mathrm{mg}, 0.284 \mathrm{mmol}, 1.1 \mathrm{eq}$.) in THF ( $1 \mathrm{~mL}$ ) was added at room temperature. After $2 \mathrm{~h}$ at room temperature and $1 \mathrm{~h}$ at $40^{\circ} \mathrm{C}$, an aqueous treatment (satured aqueous solution of $\mathrm{NaHCO}_{3}$ ) and chromatography on silica gel (elution with pentane/ $\mathrm{Et}_{2} \mathrm{O}$ : 70/30), $96 \mathrm{mg}(85 \%)$ of product 36 was obtained as a yellow oil. The two diastereomers were not separated during the chromatography, ratio $: 1 / 1$.

$\mathbf{R f}=0.27\left(\right.$ pentane $\left./ \mathrm{Et}_{2} \mathrm{O}: 70 / 30\right) . \mathbf{M p}=74^{\circ} \mathrm{C} . \mathbf{N M R}^{1} \mathbf{H}\left(300 \mathrm{MHz}, \mathrm{CDCl}_{3}\right) \delta 6.37(\mathrm{t}, 1 \mathrm{H}, J=$ $4.1 \mathrm{~Hz}), 4.73(\mathrm{~m}, 1 \mathrm{H}), 4.50(\mathrm{t}, 0.5 \mathrm{H}, J=6.2 \mathrm{~Hz}$, one diastereomer), $4.45(\mathrm{t}, 0.5 \mathrm{H}, J=6.2 \mathrm{~Hz}$, 
one diastereomer), $2.26(\mathrm{t}, 2 \mathrm{H}, J=7.0 \mathrm{~Hz}), 2.14-1.96(\mathrm{~m}, 4 \mathrm{H}), 1.89-1.64(\mathrm{~m}, 6 \mathrm{H}), 1.68(\mathrm{~s}$, $3 \mathrm{H}), 1.39$ (s, 3H), $0.13(\mathrm{~s}, 9 \mathrm{H}) . \mathbf{N M R}{ }^{13} \mathbf{C}\left(75 \mathrm{MHz}, \mathrm{CDCl}_{3}\right) \delta 135.0,124.5,124.4,110.50$, $110.45,106.7,89.7,89.6,84.8,83.71,83.67,79.7,79.6,75.4,75.3,62.0,38.2,36.3,36.2$, 27.5, 26.3, 24.1, 24.0, 19.7, 19.5, 0.1. IR $\left(\mathrm{CHCl}_{3}\right): v=3371,2941,2173,1378,1248,1210$, $1065,838 \mathrm{~cm}^{-1}$.

$(3 \mathrm{a} R *, 11 \mathrm{c} S *)-2,2-d i m e t h y l-7-(t r i m e t h y l s i l y l)-5,6,9,10,11,11 \mathrm{c}-h e x a h y d r o-4 H, 8 H-$ anthra $\left[1^{\prime}, 9^{\prime}: 1,4,3\right]$ cyclobuta[1,2-d] [1,3]dioxol-11-ol (26)

To a solution of compound $36(92 \mathrm{mg}, 0.209 \mathrm{mmol}, 1$ eq.) in dried benzene (1.8 $\mathrm{mL})$ was added $\mathrm{Pd}\left(\mathrm{PPh}_{3}\right)_{4}(24 \mathrm{mg}, 0,021 \mathrm{mmol}, 0,1$ eq. $)$ and diisopropyl amine $(0.6 \mathrm{~mL})$. The mixture was purged 5 min with argon and heated at $130^{\circ} \mathrm{C}$ under microwave irradiation during 20 min. The reaction mixture was concentrated in vacuo and the crude purified by chromatography on silica gel (elution with pentane/Et $\left.\mathrm{t}_{2} \mathrm{O}: 70 / 30\right)$ to afford $69 \mathrm{mg}(92 \%)$ of 26 as a yellow solid. The two diastereomer are not separable and are in same ratio 1:1.

$\mathbf{R f}=0.37\left(\right.$ pentane $\left./ \mathrm{Et}_{2} \mathrm{O}: 70 / 30\right) . \mathbf{M p}=74^{\circ} \mathrm{C} . \mathbf{N M R}{ }^{1} \mathbf{H}\left(200 \mathrm{MHz}, \mathrm{CDCl}_{3}\right) \delta 5.41(\mathrm{~s}, 1 \mathrm{H})$, $5.28(\mathrm{~s}, 1 \mathrm{H}), 5.02($ br t, $1 \mathrm{H}), 4.80($ br t, $1 \mathrm{H}), 3.17-3.08(\mathrm{~m}, 2 \mathrm{H}), 2.99-2.71(\mathrm{~m}, 4 \mathrm{H}), 2.44-1.88$ $(\mathrm{m}, 14 \mathrm{H}), 1.86-1.67(\mathrm{~m}, 4 \mathrm{H}), 1.64-1.59(\mathrm{~m}, 2 \mathrm{H}), 1.52(\mathrm{~s}, 3 \mathrm{H}), 1.51(\mathrm{~s}, 3 \mathrm{H}), 1.42(\mathrm{~s}, 3 \mathrm{H}), 1.42$ $(\mathrm{s}, 3 \mathrm{H}), 0.37(\mathrm{~s}, 9 \mathrm{H}), 0.37(\mathrm{~s}, 9 \mathrm{H}) . \mathbf{N M R}{ }^{13} \mathbf{C}\left(50 \mathrm{~Hz}, \mathrm{CDCl}_{3}\right) \delta 146.9,146.7,146.2,145.3$, $145.1,140.6,138.4,138.2$, 134.1, 132.3, 114.6, 114.4, 88.2, 88.1, 83.6, 82.9, 67.3, 67.0, 32.2, $31.8,31.5,31.4,28.9,28.7,28.33,28.29,22.8,20.8,19.8,3.0,2.9$. COSY, HSQC, HMBC, NOESY. MS (apparatus 3, ESI, positif ion): $359.2\left[\mathrm{MH}^{+}\right], 381.1\left[\mathrm{M}+\mathrm{Na}^{+}\right], 341.2,324.1$, 301.1, 283.1.

\section{Synthesis of 84 from 79}

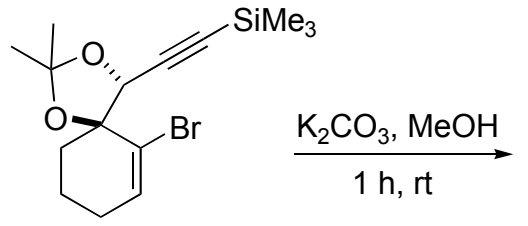

79

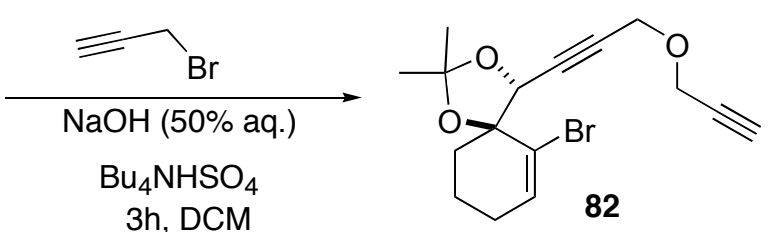

3h, DCM

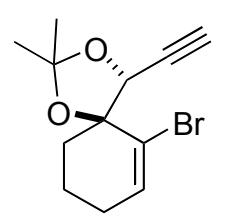

80

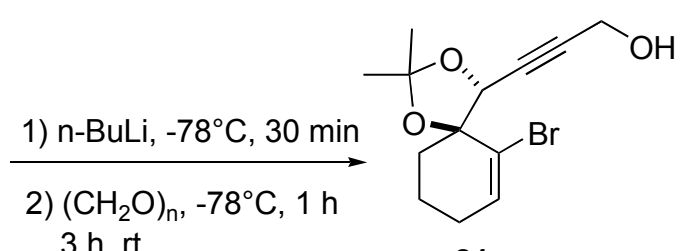

$3 \mathrm{~h}, \mathrm{rt}$

81

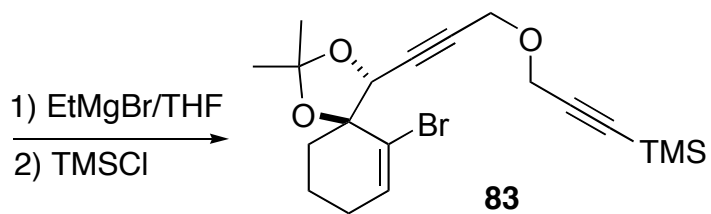

$\left(4 R^{*}, 5 S^{*}\right)$-6-bromo-4-ethynyl-2,2-dimethyl-1,3-dioxaspiro[4.5]dec-6-ene (80)

Compound 80 was prepared following the same procedure than for the preparation of 59, starting from compound $\mathbf{7 9}^{2}$ (984 mg, $2.87 \mathrm{mmol}$, 1 eq.) and $\mathrm{K}_{2} \mathrm{CO}_{3}$ (396 mg, $2.87 \mathrm{mmol}, 1$ eq.) in $\mathrm{MeOH}(15 \mathrm{~mL})$. After $1 \mathrm{~h}$ of reaction, the same aqueous treatment were applied and the crude product was purified by chromatography on silica gel (elution with heptane/Et $\mathrm{O}$ : $95 / 5)$ to give $668 \mathrm{mg}(86 \%)$ of the product 80 as a white powder. 
$\mathbf{R f}=0.39($ heptane/Et $2 \mathrm{O}: 90 / 10) .{ }^{1} \mathbf{H}$ NMR $\left(300 \mathrm{MHz}, \mathrm{CDCl}_{3}\right) \delta 6.49(\mathrm{t}, J=4.2 \mathrm{~Hz}, 1 \mathrm{H})$, $5.08(\mathrm{~d}, J=2.2 \mathrm{~Hz}, 1 \mathrm{H}), 2.63(\mathrm{~d}, J=2.2 \mathrm{~Hz}, 1 \mathrm{H}), 2.38-1.72(\mathrm{~m}, 6 \mathrm{H}), 1.52(\mathrm{~s}, 6 \mathrm{H}) .{ }^{13} \mathbf{C} \mathbf{~ N M R}$ $\left(75 \mathrm{MHz}, \mathrm{CDCl}_{3}\right) \delta$ 138.1, 122.8, 109.5, 82.1, 77.7, 77.2, 72.0, 33.9, 28.1, 27.6, 26.3, 18.9.

\section{3 -[(4R*,5S*)-6-bromo-2,2-dimethyl-1,3-dioxaspiro[4.5]dec-6-en-4-yl]prop-2-yn-1-ol (81)}

Compound 81 was prepared following the same procedure than for the preparation of $\mathbf{6 0}$, starting from compound $\mathbf{8 0}(300 \mathrm{mg}, 1.11 \mathrm{mmol}, 1$ eq.), $n$-BuLi $(0.760 \mathrm{~mL}, 1.22 \mathrm{mmol}, 1.1$ eq.) and paraformaldehyde $\left(50 \mathrm{mg}, 1.67 \mathrm{mmol}, 1.5\right.$ eq.) in THF $(10 \mathrm{~mL})$. After $1 \mathrm{~h}$ at $-78^{\circ} \mathrm{C}$ and $3 \mathrm{~h}$ at RT, an aqueous treatment and a chromatography on silica gel (elution with heptane/Et ${ }_{2} \mathrm{O}$ : 50/50), $232 \mathrm{mg}(69 \%)$ of product $\mathbf{8 1}$ was obtained as a colourless oil.

$\mathbf{R f}=0.20\left(\right.$ heptane $\left./ \mathrm{Et}_{2} \mathrm{O}: 50 / 50\right) .{ }^{1} \mathbf{H} \mathbf{N M R}\left(200 \mathrm{MHz}, \mathrm{CDCl}_{3}\right) \delta 6.50(\mathrm{t}, J=4.3 \mathrm{~Hz}, 1 \mathrm{H}), 4.73$ $(\mathrm{t}, J=1.5 \mathrm{~Hz}, 1 \mathrm{H}), 4.33(\mathrm{dd}, J=5.6 \mathrm{~Hz}, J=1.5 \mathrm{~Hz}, 2 \mathrm{H}), 2.15-1.59(\mathrm{~m}, 7 \mathrm{H}), 1.51(\mathrm{~s}, 6 \mathrm{H}) .{ }^{13} \mathbf{C}$ NMR $\left(50 \mathrm{MHz}, \mathrm{CDCl}_{3}\right) \delta 138.6,122.5,109.3,87.7,82.2,79.1,72.3,50.7,33.9,28.3,27.7$, $26.4,18.9$.

\section{$\left(4 R^{*}, 5 S^{*}\right)$-6-bromo-2,2-dimethyl-4-[3-(prop-2-ynyloxy)prop-1-ynyl]-1,3- dioxaspiro[4.5]dec-6-ene $(82)$}

Compound 82 was prepared following the same procedure than for the preparation of $\mathbf{6 1}$, starting from compound $\mathbf{8 1}$ (232 mg, $0.77 \mathrm{mmol}$, 1 eq.), propargyl bromine (363 $\mathrm{mg}, 3.08$ mmol, 4.0 eq., $80 \%$ in toluene), $n \mathrm{Bu}_{4} \mathrm{NHSO}_{4}(26 \mathrm{mg}, 0.08 \mathrm{mmol}, 0.1$ eq.) and $1.4 \mathrm{~mL}$ of solution of $\mathrm{NaOH}\left(50 \%\right.$ aq.) in $\mathrm{CH}_{2} \mathrm{Cl}_{2}(4 \mathrm{~mL})$. Aqueous treatment and chromatography on silica gel (elution with heptane/ $\left.\mathrm{Et}_{2} \mathrm{O}: 80 / 20\right)$ afford $240 \mathrm{mg}(92 \%)$ of the product 82 as a yellow oil.

$\mathbf{R f}=0.58($ heptane/Et $2 \mathrm{O}: 50 / 50) . \mathbf{N M R}^{1} \mathbf{H}\left(300 \mathrm{MHz}, \mathrm{CDCl}_{3}\right) \delta 6.49(\mathrm{t}, 1 \mathrm{H}, J=4.1 \mathrm{~Hz})$, $5.13(\mathrm{t}, 1 \mathrm{H}, J=1.7 \mathrm{~Hz}), 4.35(\mathrm{~d}, 2 \mathrm{H}, J=1.7 \mathrm{~Hz}), 4.26(\mathrm{~d}, 2 \mathrm{H}, J=2.4 \mathrm{~Hz}), 2.46(\mathrm{t}, 1 \mathrm{H}, J=$ $2.4 \mathrm{~Hz}), 2.12-1.72(\mathrm{~m}, 6 \mathrm{H}), 1.51(\mathrm{~s}, 6 \mathrm{H}) . \mathbf{N M R}{ }^{13} \mathbf{C}\left(75 \mathrm{MHz}, \mathrm{CDCl}_{3}\right) \delta 138.4,122.8,109.4$, $84.3,82.2,80.8,78.6,75.1,72.3,56.6,56.5,34.0,28.3,27.7,26.4,19.0$.

\section{[3-(\{3-[(4R*,5S*)-6-bromo-2,2-dimethyl-1,3-dioxaspiro[4.5]dec-6-en-4-yl]prop-2- ynyl\}oxy)prop-1-ynyl](trimethyl)silane (83)}

Compound 83 was prepared following the same procedure than for the preparation of $\mathbf{1}$, starting from compound $82(240 \mathrm{mg}, 0.710 \mathrm{mmol}, 1$ eq.), EtMgBr $(0.4 \mathrm{~mL}, 0.78 \mathrm{mmol}, 1.1$ eq.) and TMSCl (0.1 mL, $0.780 \mathrm{mmol}, 1.1$ eq.) in anhydrous THF (5.5 mL). After an aqueous treatment and chromatography on silica gel (elution with heptane/Et $\left.{ }_{2} \mathrm{O}: 95 / 5\right), 151 \mathrm{mg}(52 \%)$ of product $\mathbf{8 3}$ was obtained as a yellow oil.

$\mathbf{R f}=0.46\left(\right.$ heptane $\left./ \mathrm{Et}_{2} \mathrm{O}: 80 / 20\right) . \mathbf{N M R}^{1} \mathbf{H}\left(300 \mathrm{MHz}, \mathrm{CDCl}_{3}\right) \delta 6.49(\mathrm{t}, 1 \mathrm{H}, J=4.2 \mathrm{~Hz}), 4.76$ (t, $1 \mathrm{H}, J=1.5 \mathrm{~Hz}), 4.33(\mathrm{~d}, 2 \mathrm{H}, J=1.5 \mathrm{~Hz}), 4.24(\mathrm{~s}, 2 \mathrm{H}), 2.34-1.72(\mathrm{~m}, 6 \mathrm{H}), 1.51(\mathrm{~s}, 6 \mathrm{H})$, 0.19 (s, 9H). NMR ${ }^{13} \mathbf{C}\left(75 \mathrm{MHz}, \mathrm{CDCl}_{3}\right) \delta 138.5,122.8,109.4,100.2,92.2,84.5,82.3,80.7$, $72.4,57.3,56.6,34.1,28.3,27.8,26.5,19.1,-0.3$.

$\left(1 S^{*}\right)-2-b r o m o-1-\left(\left(1 R^{*}\right)-1-h y d r o x y-4-\{[3-(t r i m e t h y l s i l y l) p r o p-2-y n y l] o x y\}\right.$ but-2ynyl)cyclohex-2-en-1-ol (84)

To a solution of compound 83 (80 mg, 0.19 mmol, 1 eq.) in $\mathrm{Et}_{2} \mathrm{O}(1 \mathrm{~mL})$ was added a solution of $\mathrm{HCl}\left(6 \mathrm{~mL}, 6 \mathrm{mmol}, 32\right.$ eq., $2 \mathrm{M}$ in $\left.\mathrm{Et}_{2} \mathrm{O}\right)$ at $0^{\circ} \mathrm{C}$. After 8 days at room temperature, the 
crude mixture was extracted with $\mathrm{Et}_{2} \mathrm{O}$, washed with water, dried over $\mathrm{Na}_{2} \mathrm{SO}_{4}$, filtrated and concentrated in vacuo. A chromatography on silica gel (elution with heptane/ $\mathrm{Et}_{2} \mathrm{O}: 50 / 50$ ), 31 mg (44\%) of product 84 was obtained as a colorless oil.

$\mathbf{R f}=0.27\left(\right.$ heptane $\left./ \mathrm{Et}_{2} \mathrm{O}: 50 / 50\right) . \mathbf{N M R}^{1} \mathbf{H}\left(300 \mathrm{MHz}, \mathrm{CDCl}_{3}\right) \delta 6.41(\mathrm{dd}, 1 \mathrm{H}, J=3.2 \mathrm{~Hz}, J=$ $5.2 \mathrm{~Hz}), 4.84(\mathrm{~s}, 1 \mathrm{H}), 4.29(\mathrm{~d}, 2 \mathrm{H}, J=1.9 \mathrm{~Hz}), 4.23(\mathrm{~s}, 2 \mathrm{H}), 2.50($ br s, $1 \mathrm{H}, \mathrm{OH}), 2.45$ ( br s, 1H, OH) 2.27-1.99 (m, 4H), 1.91-1.60 (m, 2H), $0.19(\mathrm{~s}, 9 \mathrm{H}) . \mathbf{N M R}{ }^{13} \mathbf{C}\left(75 \mathrm{MHz}, \mathrm{CDCl}_{3}\right) \delta$ $136.5,125.1,100.6,92.2,83.9,82.8,75.4,69.8,57.2,56.6,31.6,28.3,18.2,-0.2$.

\section{Synthesis of 85 from 2}

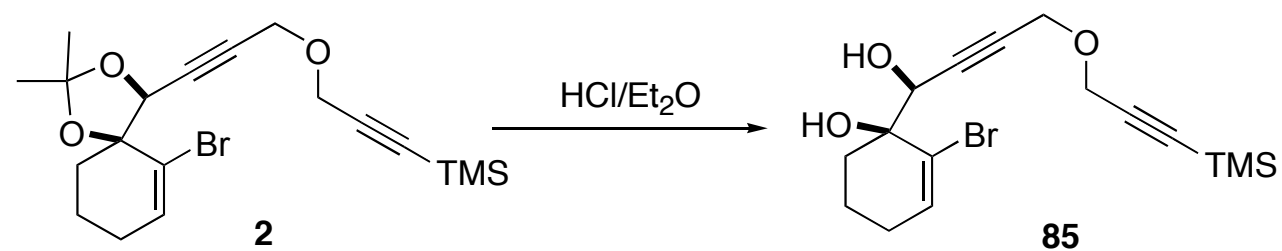

\section{(1S*)-2-bromo-1-((1S*)-1-hydroxy-4-\{[3-(trimethylsilyl)prop-2-ynyl]oxy\}but-2-ynyl)} cyclohex-2-en-1-ol (85)

To a solution of compound 2 (200 $\mathrm{mg}, 0.486 \mathrm{mmol}, 1$ eq.) in $\mathrm{Et}_{2} \mathrm{O}(1 \mathrm{~mL})$ was added a solution of $\mathrm{HCl}\left(6 \mathrm{~mL}, 12 \mathrm{mmol}, 24.7\right.$ eq., $2 \mathrm{M}$ in $\left.\mathrm{Et}_{2} \mathrm{O}\right)$ at $0^{\circ} \mathrm{C}$. After 8 days at room temperature, the crude mixture was extracted with $\mathrm{Et}_{2} \mathrm{O}$, washed with water, dried over $\mathrm{Na}_{2} \mathrm{SO}_{4}$, filtrated and concentrated in vacuo. After chromatography on silica gel (elution with heptane/ $\left.\mathrm{Et}_{2} \mathrm{O}: 80 / 20\right), 86 \mathrm{mg}(48 \%)$ of product was obtained as a yellow oil

$\mathbf{R f}=0.33$ (heptane $\left./ \mathrm{Et}_{2} \mathrm{O}: 50 / 50\right) . \mathbf{N M R}^{1} \mathbf{H}\left(300 \mathrm{MHz}, \mathrm{CDCl}_{3}\right) \delta 6.44(\mathrm{dd}, 1 \mathrm{H}, J=3.1 \mathrm{~Hz}, J=$ $5.1 \mathrm{~Hz}), 4.70(\mathrm{~s}, 1 \mathrm{H}), 4.32(\mathrm{~d}, 2 \mathrm{H}, J=1.7 \mathrm{~Hz}), 4.26(\mathrm{~s}, 2 \mathrm{H}), 2.57$ (br s, 1H, OH), 2.33 ( br s, $1 \mathrm{H}, \mathrm{OH}) 2.23-2.00(\mathrm{~m}, 4 \mathrm{H}), 1.86-1.75(\mathrm{~m}, 2 \mathrm{H}), 0.19(\mathrm{~s}, 9 \mathrm{H})$.

\section{Synthesis of 28 from 84 and 85}

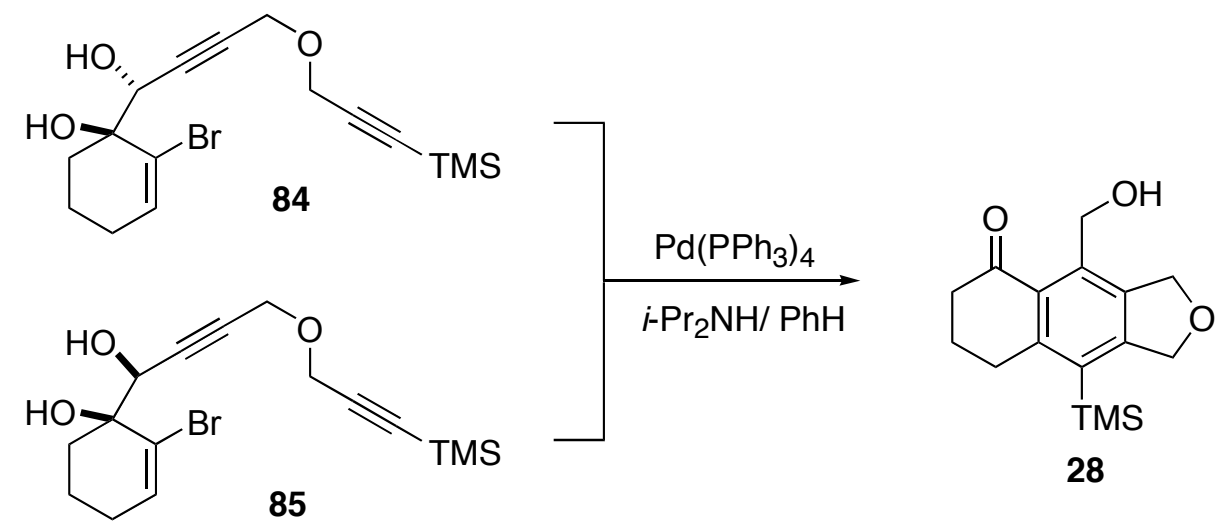

\section{4-(hydroxymethyl)-9-(trimethylsilyl)-3,6,7,8-tetrahydronaphtho[2,3-c]furan-5(1H)-one}

\section{(28)}

To a solution of compound $\mathbf{8 5}(86 \mathrm{mg}, 0.23 \mathrm{mmol}, 1$ eq.) in anhydrous benzene $(1.5 \mathrm{~mL})$ was added $\mathrm{Pd}\left(\mathrm{PPh}_{3}\right)_{4}(27 \mathrm{mg}, 0,023 \mathrm{mmol}, 0,1$ eq. $)$ and diisopropyl amine $(0.8 \mathrm{~mL})$. The mixture was purged 5 min with argon and heated during $20 \mathrm{~min}$ at $130^{\circ} \mathrm{C}$ under microwave irradiation. The reaction mixture was concentrated in vacuo and purified by chromatography on silica gel (elution with heptane/Et $\mathrm{E}_{2} \mathrm{O}$ : 1/2) to afford $31 \mathrm{mg}$ (46\%) of 28 as a yellow oil. 
To a solution of compound 84 (31 $\mathrm{mg}, 0.083 \mathrm{mmol}, 1$ eq.) in anhydrous benzene $(0.6 \mathrm{~mL})$ was added $\mathrm{Pd}\left(\mathrm{PPh}_{3}\right)_{4}(10 \mathrm{mg}, 0,008 \mathrm{mmol}, 0,1$ eq. $)$ and diisopropyl amine $(0.2 \mathrm{~mL})$. The mixture was purged 5 min with argon and heated during $20 \mathrm{~min}$ at $130^{\circ} \mathrm{C}$ under microwave irradiation. The reaction mixture was concentrated in vacuo and purified by chromatography on silica gel (elution with pentane/Et $\left.{ }_{2} \mathrm{O}: 1 / 1\right)$ to afford $14 \mathrm{mg}(58 \%)$ of $\mathbf{2 8}$ as a yellow oil.

$\mathbf{R f}=0.16\left(\right.$ heptane $\left./ \mathrm{Et}_{2} \mathrm{O}: 2 / 1\right) . \mathbf{N M R}^{1} \mathbf{H}\left(300 \mathrm{MHz}, \mathrm{CDCl}_{3}\right) \delta 5.21-5.18(\mathrm{~m}, 4 \mathrm{H}), 4.49(\mathrm{~d}, 2 \mathrm{H}$, $J=7.4 \mathrm{~Hz}), 4.31(\mathrm{t}, 1 \mathrm{H}, J=7.4 \mathrm{~Hz}), 3.05(\mathrm{t}, 2 \mathrm{H}, J=6.3 \mathrm{~Hz}), 2.73(\mathrm{t}, 2 \mathrm{H}, J=6.3 \mathrm{~Hz}), 2.07$ (quint, $2 \mathrm{H}, J=6.3 \mathrm{~Hz}), 0.39(\mathrm{~s}, 9 \mathrm{H}) . \mathbf{N M R}{ }^{13} \mathbf{C}\left(75 \mathrm{MHz}, \mathrm{CDCl}_{3}\right) \delta 202.7,152.3,150.8$, 137.6, 136.3, 131.78, 131.76, 75.6, 72.0, 61.5, 40.0, 32.1, 22.6, 1.8. MS (API-ES) : 313.1 [M+Na], 329. 1 [M+K]. HRMS (ESI, positive ion): calc'd for $\left(\mathrm{C}_{16} \mathrm{H}_{22} \mathrm{O}_{3} \mathrm{NaSi}\right)^{+} 313.1227$; found 313.1230 .

\section{Synthesis of 11 from 86}

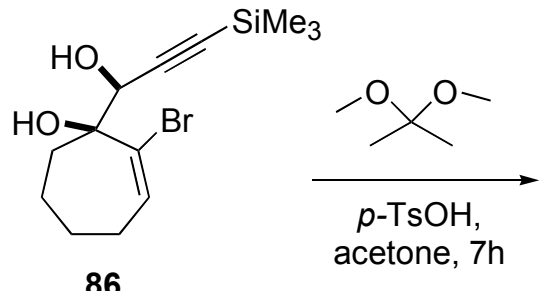

86

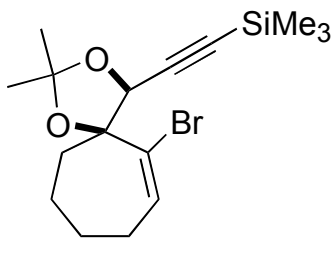

31

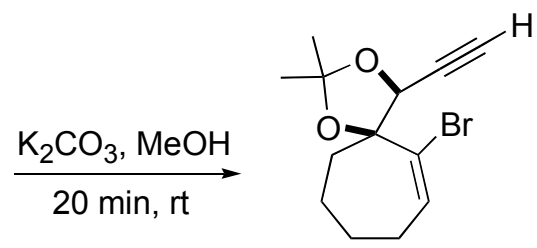

87
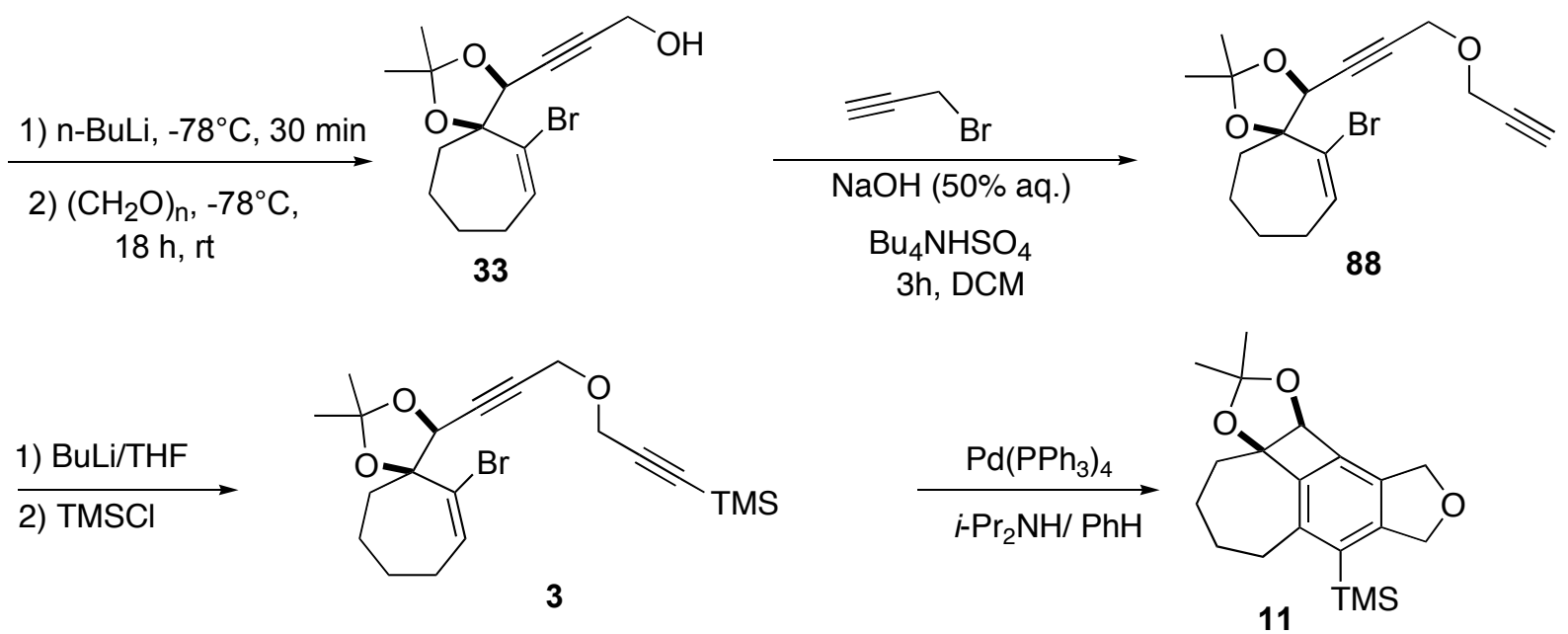

$\{[(4 S *, 5 S *)-6-b r o m o-2,2-d i m e t h y l-1,3-d i o x a s p i r o[4.6]$ undec-6-en-4-yl]ethynyl $\}$ (trimethyl)silane (31)

Compound 31 was prepared following the same procedure than for the preparation of 58, starting from compound $\mathbf{8 6}^{2}$ ( $2 \mathrm{~g}, 6.30 \mathrm{mmol}, 1$ eq.), 2,2-dimethoxypropane (20 mL, 169.6 mmol, 27 eq.), and $p$-TsOH (200 mg, $1.05 \mathrm{mmol}, 0.17$ eq.) in acetone $(50 \mathrm{~mL})$ during $7 \mathrm{~h}$. The same aqueous treatment was applied and the crude product was purified by chromatography on silica gel (elution with pentane/Et $\left.{ }_{2} \mathrm{O}: 98 / 2\right)$ to give $2.1 \mathrm{~g}(93 \%)$ of the product $\mathbf{3 1}$ as a white powder.

$\mathbf{R f}=0.16\left(\right.$ pentane $\left./ \mathrm{Et}_{2} \mathrm{O}: 98 / 2\right) . \mathbf{M p}=44^{\circ} \mathrm{C} .{ }^{1} \mathbf{H}$ NMR $\left(200 \mathrm{MHz}, \mathrm{CDCl}_{3}\right) \delta 6.54(\mathrm{t}, J=6.84$ $\mathrm{Hz}, 1 \mathrm{H}), 4.82(\mathrm{~s}, 1 \mathrm{H}), 2.28-1.5(\mathrm{~m}, 8 \mathrm{H}), 1.70(\mathrm{~s}, 3 \mathrm{H}), 1.39(\mathrm{~s}, 3 \mathrm{H}), 0.17(\mathrm{~s}, 9 \mathrm{H}) .{ }^{13} \mathbf{C}$ NMR $(50$ $\left.\mathrm{MHz}, \mathrm{CDCl}_{3}\right) \delta 135.4,129.6,110.8,100.2,94.2,88.9,73.7,37.6,27.3,26.4,24.5,21.7,-0.5$. IR $\left(\mathrm{CHCl}_{3}\right)$ vv. 3053, 2938, 2857, 1457, 1379, 1249, 1208, 1188, 1163, 1096, 1069, 1042, 
$980,939,893,843,760,738,704,654,559,524,407 \mathrm{~cm}^{-1}$.

$\left(4 S^{*}, 5 S^{*}\right)-6-b r o m o-4-e t h y n y l-2,2-d i m e t h y l-1,3-d i o x a s p i r o[4.6]$ undec-6-ene (87)

Compound 87 was prepared following the same procedure than for the preparation of 59, starting from compound 31 (2.89 g, 8.09 mmol, 1 eq.) and $\mathrm{K}_{2} \mathrm{CO}_{3}$ (1.12 g, 8.09 mmol, 1 eq.) in $\mathrm{MeOH}(40 \mathrm{~mL})$. After $20 \mathrm{~min}$ of reaction, $2.25 \mathrm{~g}(97 \%)$ of product 87 was obtained as a white powder, and used without purification.

$\mathbf{R f}=0.58$ (heptane $\left./ \mathrm{Et}_{2} \mathrm{O}: 70 / 30\right) . \mathbf{M p}=78^{\circ} \mathrm{C} .{ }^{1} \mathbf{H} \mathbf{N M R}\left(200 \mathrm{MHz}, \mathrm{CDCl}_{3}\right) \delta 6.59(\mathrm{t}, J=6,6$ $\mathrm{Hz}, 1 \mathrm{H}), 4.86(\mathrm{~d}, J=2,2 \mathrm{~Hz}, 1 \mathrm{H}), 2.65(\mathrm{~d}, J=2,44 \mathrm{~Hz}, 1 \mathrm{H}), 2.30-1.54(\mathrm{~m}, 8 \mathrm{H}), 1.72(\mathrm{~s}, 3 \mathrm{H})$, $1.42(\mathrm{~s}, 3 \mathrm{H}) .{ }^{13} \mathbf{C}$ NMR $\left(50 \mathrm{MHz}, \mathrm{CDCl}_{3}\right) \delta 136.3,129.2,111.0,88.6,78.7,77.2,73.4,37.7$, 27.4, 26.5, 26.4, 24.6, 21.7. IR $\left(\mathrm{CHCl}_{3}\right) v=3853,3751,3648,3283,2938,2360,1684,1653$, $1558,1541,1507,1457,1375,1261,1209,1163,1068,1040,866,669,518,419 \mathrm{~cm}^{-1}$.

\section{3-[(4S*,5S*)-6-bromo-2,2-dimethyl-1,3-dioxaspiro[4.6] undec-6-en-4-yl]prop-2-yn-1-ol} (33)

Compound 33 was prepared following the same procedure than for the preparation of $\mathbf{6 0}$, starting from compound 87 (2.14 g, $7.50 \mathrm{mmol}, 1$ eq.), $n$-BuLi (5.1 mL, 8.25 mmol, 1.1 eq., 1.6 $\mathrm{M}$ in hexane) and paraformaldehyde (356 mg, $11.25 \mathrm{mmol}, 1.5 \mathrm{eq}$.$) in THF (63 mL).$ After $18 \mathrm{~h}$ of reaction, an aqueous treatment and a chromatography on silica gel (elution with pentane/AcOEt: 80/20), $1.715 \mathrm{~g}$ (73\%) of product 33 was obtained as a white solid.

$\mathbf{R f}=0.24$ (pentane/AcOEt: 80/20). $\mathbf{M p}=72^{\circ} \mathrm{C} .{ }^{1} \mathbf{H}$ NMR $\left(200 \mathrm{MHz}, \mathrm{CDCl}_{3}\right) \delta 6.57(\mathrm{t}, J=$ $6.84 \mathrm{~Hz}, 1 \mathrm{H}), 4.88(\mathrm{t}, J=1.7 \mathrm{~Hz}, 1 \mathrm{H}), 4.33(\mathrm{~d}, J=2.44 \mathrm{~Hz}, 2 \mathrm{H}), 2.29-1.64(\mathrm{~m}, 9 \mathrm{H}), 1.71$ (s, $3 \mathrm{H}), 1.41(\mathrm{~s}, 3 \mathrm{H}) .{ }^{13} \mathbf{C}$ NMR $\left(50 \mathrm{MHz}, \mathrm{CDCl}_{3}\right) \delta 136.3,129.2,110.9,88.7,87.3,80.6,73.6$, 51.1, 37.6, 27.4, 26.4, 24.7, 21.6. IR $\left(\mathrm{CHCl}_{3}\right) v=3414,2986,2937,2864,2183,2144,1457$, $1380,1275,1261,1209,1162,1043,898,867,764,750,559,430 \mathrm{~cm}^{-1}$.

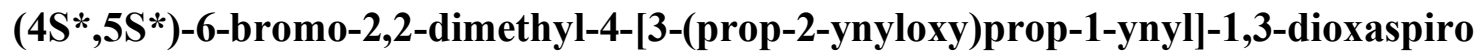 [4.6]undec-6-ene (88)}

Compound 88 was prepared following the same procedure than for the preparation of $\mathbf{6 1}$, starting from compound 33 (315 mg, $1.0 \mathrm{mmol}$, 1 eq.), propargyl bromine (520 mg, 3.5 mmol, 3.2 eq., $80 \%$ in toluene), $n \mathrm{Bu}_{4} \mathrm{NHSO}_{4}(34 \mathrm{mg}, 0.1 \mathrm{mmol}, 0.1$ eq. cat.) and $2 \mathrm{~mL}$ of solution of $\mathrm{NaOH}\left(50 \%\right.$ aq.) in $\mathrm{CH}_{2} \mathrm{Cl}_{2}(6 \mathrm{~mL})$. Aqueous treatment and chromatography on silica gel (elution with hexane/Et $\left.{ }_{2} \mathrm{O}: 50 / 50\right)$ afford $320 \mathrm{mg}(91 \%)$ of the product 88 as a yellow oil.

$\mathbf{R f}=0.60$ (hexane $\left./ \mathrm{Et}_{2} \mathrm{O}: 50 / 50\right) . \mathbf{N M R}^{1} \mathbf{H}\left(300 \mathrm{MHz}, \mathrm{CDCl}_{3}\right) \delta 6.57(\mathrm{t}, 1 \mathrm{H}, J=6.4 \mathrm{~Hz}), 4.90$ $(\mathrm{s}, 1 \mathrm{H}), 4.35$ (br s, 2H), $4.31(\mathrm{dd}, 2 \mathrm{H}, J=2.5 \mathrm{~Hz}, J=5.7 \mathrm{~Hz}), 2.44(\mathrm{t}, 1 \mathrm{H}, J=2.5 \mathrm{~Hz})$, 2.33-1.97 (m, 4H), 1.90-1.55 (m, 7H), $1.41(\mathrm{~s}, 3 \mathrm{H})$. NMR ${ }^{13} \mathbf{C}\left(50 \mathrm{MHz}, \mathrm{CDCl}_{3}\right) \delta 136.2$, $129.4,110.8,88.5,84.0,81.1,78.9,74.9,73.7,56.6,56.3,37.5,27.3,26.3,24.6,21.5$. IR (FTIR, film): $v=3409,3287,2986,2938,2861,2363,2117,1624,1447,1378,1252,1209$, $1161,1083,1045,936,896,869,788,668 \mathrm{~cm}^{-1}$.

[3-(\{3-[(4S*,5S*)-6-bromo-2,2-dimethyl-1,3-dioxaspiro[4.6]undec-6-en-4-yl]prop-2 ynyl\}oxy)prop-1-ynyl](trimethyl)silane (3)

Compound 3 was prepared following the same procedure than for the preparation of $\mathbf{1}$, starting from compound $\mathbf{8 8}$ (290 mg, $0.821 \mathrm{mmol}, 1$ eq.), $n$-BuLi (0.72 mL, $0.862 \mathrm{mmol}, 1.05$ 
eq.) and TMSCl (97 mg, $0.895 \mathrm{mmol}, 1.09$ eq.) in anhydrous THF (10 mL). After an aqueous treatment and chromatography on silica gel (elution with hexane/Et $\left.{ }_{2} \mathrm{O}: 80 / 20\right), 290 \mathrm{mg}(83 \%)$ of product $\mathbf{3}$ was obtained as a yellow oil.

$\mathbf{R f}=0.58\left(\right.$ hexane/Et $\left.\mathrm{E}_{2} \mathrm{O}: 70 / 30\right) . \mathbf{N M R}{ }^{1} \mathbf{H}\left(300 \mathrm{MHz}, \mathrm{CDCl}_{3}\right) \delta 6.57(\mathrm{t}, 1 \mathrm{H}, J=6.6 \mathrm{~Hz}), 4.89$ (br s, 1H), 4.33 (br s, 2H), $4.29(\mathrm{~d}, 2 \mathrm{H}, J=2.9 \mathrm{~Hz}), 2.35-1.95(\mathrm{~m}, 4 \mathrm{H}), 1.90-1.52(\mathrm{~m}, 7 \mathrm{H})$, $1.41(\mathrm{~s}, 3 \mathrm{H}), 0.17(\mathrm{~s}, 9 \mathrm{H}) . \mathbf{N M R}{ }^{13} \mathbf{C}\left(50 \mathrm{MHz}, \mathrm{CDCl}_{3}\right) \delta 136.1,129.4,110.8,100.6,91.9$, $88.5,84.2,81.6,73.7,57.0,56.5,37.6,26.3,24.6,21.5,-0.2$. IR (FTIR, film) : $v=2942$, $2861,2175,1624,1448,1377,1347,1252,1209,1162,1084,1046,1000,895,848,763,703$ $\mathrm{cm}^{-1}$.

$\left[\left(5 \mathrm{bR}^{*}, 8 \mathrm{aS} *\right)-7,7-\right.$ dimethyl-1,8a-dihydro-3H-5,5b-butano[1,3]dioxolo[3,4]cyclobuta[1,2-e ][2]benzofuran-4-yl](trimethy1)silane (11)

Compound 11 was prepared following the same procedure than for the preparation of $\mathbf{1 0}$, starting from compound 3 (81 mg, $0.190 \mathrm{mmol}, 1$ eq.), $\mathrm{Pd}\left(\mathrm{PPh}_{3}\right)_{4}(24 \mathrm{mg}, 0,021 \mathrm{mmol}, 0,1$ eq.) and diisopropyl amine $(0.6 \mathrm{~mL})$ in benzene $(1.4 \mathrm{~mL})$. After an aqueous treatment and chromatography on silica gel (elution with heptane/Et ${ }_{2} \mathrm{O}$ : 90/10), $59 \mathrm{mg}(89 \%$ ) of product 11 was obtained as a yellow oil.

$\mathbf{R f}=0.44\left(\right.$ heptane $\left./ \mathrm{Et}_{2} \mathrm{O}: 80 / 20\right) . \mathbf{M P}=110^{\circ} \mathrm{C} . \mathbf{~ N M R}^{1} \mathbf{H}\left(300 \mathrm{MHz}, \mathrm{CDCl}_{3}\right) \delta 5.34(\mathrm{~s}, 1 \mathrm{H})$, 5.21-4.87 (m, 4H), 3.14-2.74 (m, 2H), 2.39-1.99 (m, 4H), 1.92-1.70 (m, 1H), 1.45-1.20 (m, $1 \mathrm{H}), 1.48(\mathrm{~s}, 3 \mathrm{H}), 1.08(\mathrm{~s}, 3 \mathrm{H}), 0.33(\mathrm{~s}, 9 \mathrm{H}) . \mathbf{N M R}{ }^{13} \mathbf{C}\left(75 \mathrm{MHz}, \mathrm{CDCl}_{3}\right) \delta 148.0,146.9$, 144.1, 137.6, 132.2, 130.3, 115.3, 92.8, 84.1, 74.9, 70.7, 34.9, 34.1, 29.5, 29.1, 28.0, 27.9, 2.0. COSY, HSQC, HMBC, NOESY. IR (FTIR, film) : $v=2929,2852,2361,2247,1605$, $1441,1375,1309,1252,1204,1143,1106,1066,1022,896,844,760,733,693,653 \mathrm{~cm}^{-1}$. GC-MS (apparatus 1) : $344.2\left[\mathrm{M}^{+\bullet}\right], 286.2,257.2,73.1$.

\section{Synthesis of 14 from 33}
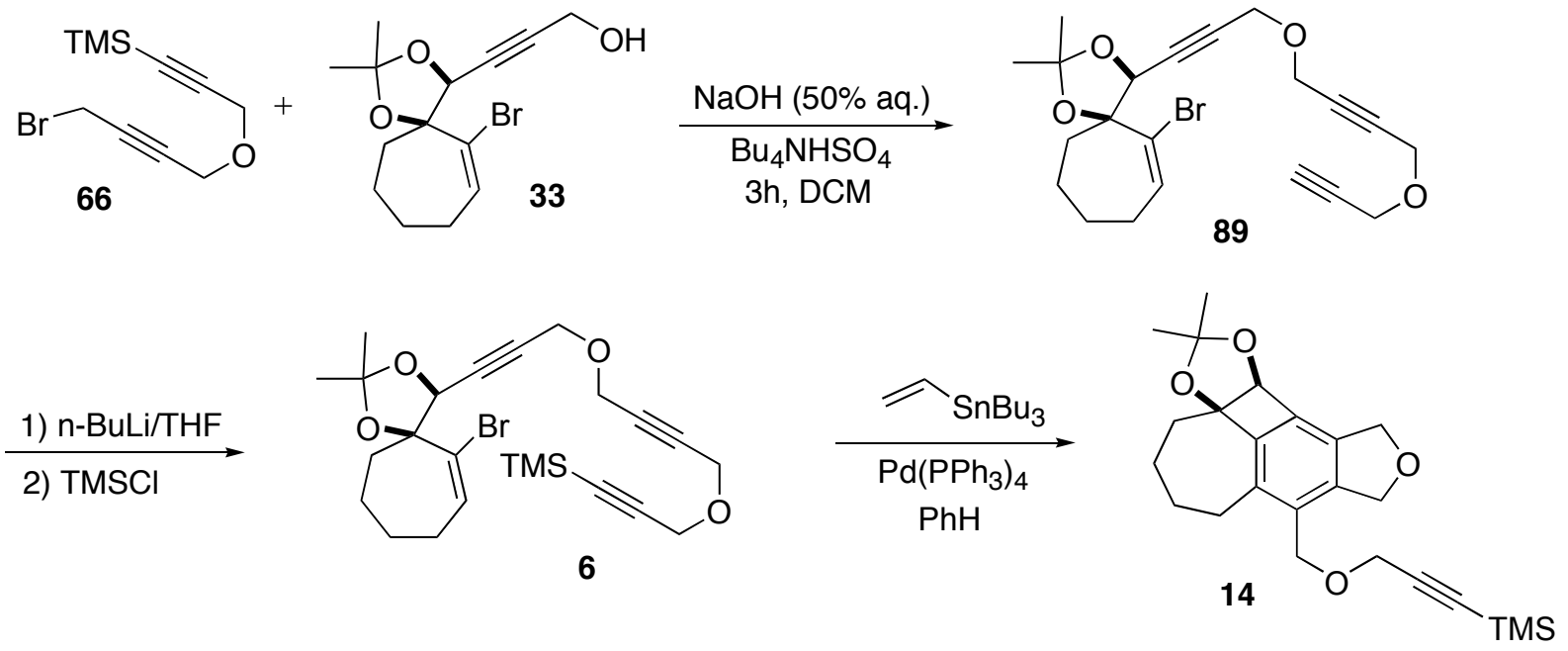

$\left(4 S^{*}, 5 \mathrm{~S} *\right)$-6-bromo-2,2-dimethyl-4-(3-\{[4-(prop-2-ynyloxy)but-2-ynyl]oxy\}prop-1-ynyl)-1 ,3-dioxaspiro[4.6]undec-6-ene (89)

To a solution of compound 33 (370 mg, $1.1 \mathrm{mmol}, 1$ eq.) in $\mathrm{CH}_{2} \mathrm{Cl}_{2}(6 \mathrm{~mL})$ was added compound 66 (910 mg, $3.5 \mathrm{mmol}, 3.2$ eq.), $n$-Bu $\mathrm{NHSO}_{4}(34 \mathrm{mg}, 0.1 \mathrm{mmol}, 0.1$ eq.) and 2 $\mathrm{mL}$ of solution of $\mathrm{NaOH}$ ( $50 \%$ aq.). After $6 \mathrm{~h}$ at $\mathrm{RT}$, the produc twas extracted with $\mathrm{CH}_{2} \mathrm{Cl}_{2}$ 
$(3 \times 10 \mathrm{~mL})$ and the combined organic layers were washed with brine, dried over $\mathrm{Na}_{2} \mathrm{SO}_{4}$ and concentrated in vacuo. The crude product was purified by chromatography on silica gel (elution with hexane/Et $\left.{ }_{2} \mathrm{O}: 80 / 20\right)$ to afford $300 \mathrm{mg}(64 \%)$ of the product 89 as a yellow oil.

$\mathbf{R f}=0.55$ (hexane/Et $\left.{ }_{2} \mathrm{O}: 50 / 50\right) . \mathbf{N M R}^{1} \mathbf{H}\left(300 \mathrm{MHz}, \mathrm{CDCl}_{3}\right) \delta 6.57(\mathrm{t}, 1 \mathrm{H}, J=6.9 \mathrm{~Hz}), 4.89$ $(\mathrm{s}, 1 \mathrm{H}), 4.41-4.21(\mathrm{~m}, 8 \mathrm{H}), 2.45(\mathrm{t}, 1 \mathrm{H}, J=2.5 \mathrm{~Hz}), 2.31-1.97(\mathrm{~m}, 4 \mathrm{H}), 1.89-1.54(\mathrm{~m}, 4 \mathrm{H})$, $1.71(\mathrm{~s}, 3 \mathrm{H}), 1.41(\mathrm{~s}, 3 \mathrm{H}) . \mathbf{N M R}{ }^{13} \mathbf{C}\left(50 \mathrm{MHz}, \mathrm{CDCl}_{3}\right) \delta 136.0,129.5,110.9,88.6,84.1$, $82.3,81.9,78.2,75.0,73.7,56.7,56.54,56.49,37.6,27.4,26.4(2 \mathrm{C}), 24.6,21.7$.

\section{(3-\{[4-(\{3-[(4S*,5S*)-6-bromo-2,2-dimethyl-1,3-dioxaspiro[4.6] undec-6-en-4-yl]prop-2-y} nyl\}oxy)but-2-ynyl]oxy\}prop-1-ynyl)(trimethyl)silane (6)

Compound $\mathbf{6}$ was prepared following the same procedure than for the preparation of $\mathbf{1}$, starting from compound $\mathbf{8 9}$ (150 mg, $0.36 \mathrm{mmol}, 1$ eq.), $n$-BuLi $(0.3 \mathrm{~mL}, 0.37 \mathrm{mmol}, 1.05$ eq.) and TMSCl (42 mg, $0.39 \mathrm{mmol}, 1.09$ eq.) in anhydrous THF (7 mL). After an aqueous treatment and chromatography on silica gel (elution with hexane/Et $\left.{ }_{2} \mathrm{O}: 85 / 15\right), 112 \mathrm{mg}(63 \%)$ of product 6 was obtained as a yellow oil.

$\mathbf{R f}=0.55$ (hexane $\left./ \mathrm{Et}_{2} \mathrm{O}: 50 / 50\right) . \mathbf{N M R}^{1} \mathbf{H}\left(300 \mathrm{MHz}, \mathrm{CDCl}_{3}\right) \delta 6.55(\mathrm{t}, 1 \mathrm{H}, J=6.9 \mathrm{~Hz}), 4.87$ $(\mathrm{s}, 1 \mathrm{H}), 4.37-4.32(\mathrm{~m}, 4 \mathrm{H}), 4.27(\mathrm{~s}, 2 \mathrm{H}), 4.22(\mathrm{~s}, 2 \mathrm{H}), 2.29-1.95(\mathrm{~m}, 4 \mathrm{H}), 1.64-1.56(\mathrm{~m}, 4 \mathrm{H})$, $1.69(\mathrm{~s}, 3 \mathrm{H}), 1.39(\mathrm{~s}, 3 \mathrm{H}), 0.17(\mathrm{~s}, 9 \mathrm{H}) . \mathbf{N M R}{ }^{13} \mathbf{C}\left(75 \mathrm{MHz}, \mathrm{CDCl}_{3}\right) \delta 136.1,129.3,110.7$, 100.4, 92.0, 88.5 , 84.0, 82.0, 81.7, 73.6, 57.2, 56.6, 56.5, 56.4, 37.5, 27.2, 26.3, 24.5, 21.5, -0.3. IR (film): $v=2942,2858,2360,2338,2251,2175,1445,1377,1346,1251,1209,1161$, $1120,1078,1046,999,898,848 \mathrm{~cm}^{-1}$.

\section{(3-\{[(5bR*,8aS*)-7,7-dimethyl-1,8a-dihydro-3H-5,5b-butano[1,3]dioxolo[3,4]cyclobuta[1 ,2-e][2]benzofuran-4-yl]methoxy\}prop-1-ynyl)(trimethyl)silane (14)}

To a solution of compound 6 (100 $\mathrm{mg}, 0.203 \mathrm{mmol}, 1$ eq.) in benzene (10 mL) was added tributylstannylethylene (93 mg, $0.293 \mathrm{mmol}, 1.3$ eq.) and $\mathrm{Pd}\left(\mathrm{PPh}_{3}\right)_{4}(27 \mathrm{mg}, 0.030 \mathrm{mmol}, 0.1$ eq.). After $5 \mathrm{~h}$ at $90^{\circ} \mathrm{C}$, the crude mixture was evaporated in vacuo and a chromatography on silica gel (elution with hexane/Et $\left.\mathrm{t}_{2} \mathrm{O}: 80 / 20\right)$, afford $27 \mathrm{mg}(32 \%)$ of compound 14 as yellow oil.

$\mathbf{R f}=0.47$ (hexane/Et $\left.\mathrm{Et}_{2} \mathrm{O}: 50 / 50\right) . \mathbf{N M R}^{1} \mathbf{H}\left(300 \mathrm{MHz}, \mathrm{CDCl}_{3}\right) \delta 5.33(\mathrm{~s}, 1 \mathrm{H}), 5.21-4.95(\mathrm{~m}$, $4 \mathrm{H}), 4.56(\mathrm{~d}, 1 \mathrm{H}, \mathrm{J}=11.0 \mathrm{~Hz}), 4.48(\mathrm{~d}, 1 \mathrm{H}, \mathrm{J}=11.0 \mathrm{~Hz}), 4.18(\mathrm{~d}, 1 \mathrm{H}, \mathrm{J}=11.8 \mathrm{~Hz}), 4.08(\mathrm{~d}$, $1 \mathrm{H}, \mathrm{J}=11.8 \mathrm{~Hz}), 3.16-2.97(\mathrm{dd}, 1 \mathrm{H}, J=4.2 \mathrm{~Hz}, J=13.4 \mathrm{~Hz}), 2.88-2.63(\mathrm{~m}, 1 \mathrm{H}), 2.37-1.98$ $(\mathrm{m}, 5 \mathrm{H}), 1.93-1.49(\mathrm{~m}, 1 \mathrm{H}), 1.48(\mathrm{~s}, 3 \mathrm{H}), 1.06(\mathrm{~s}, 3 \mathrm{H}), 0.21(\mathrm{~s}, 9 \mathrm{H}) . \mathbf{N M R}{ }^{13} \mathbf{C}(75 \mathrm{MHz}$, $\left.\mathrm{CDCl}_{3}\right) \delta 148.8,142.2,137.6,137.0,131.8,129.1,115.3,101.3,92.4,92.0,83.8,73.0,71.7$, 65.6, 57.9, 34.9, 29.5, 29.2, 28.9, 27.9, 27.6, -0,2. IR (film): $v=2930,2853,2362,1597$, 1441, 1374, 1251, 1202, 1145, 1073, 999, $846 \mathrm{~cm}^{-1}$. COSY, HSQC, HMBC, NOESY.

\section{Synthesis of 16 from 33}




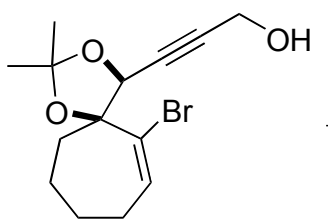

33

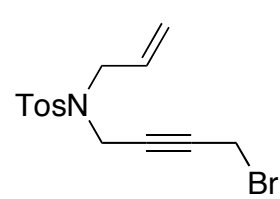

73

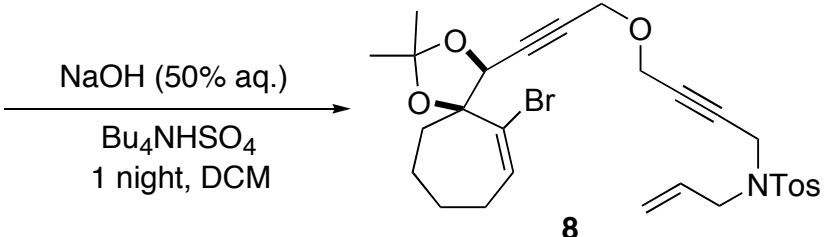

8

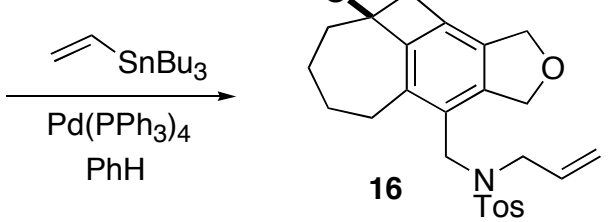

\section{$\mathrm{N}$-allyl-N-[4-(\{3-[(4S*,5S*)-6-bromo-2,2-dimethyl-1,3-dioxaspiro[4.6] undec-6-en-4yl]pro p -2-ynyl\}oxy)but-2-ynyl]-4-methylbenzenesulfonamide (8)}

Compound 8 was prepared following the same procedure than for the preparation of $\mathbf{6 1}$, starting from compound 33 (400 mg, $1.269 \mathrm{mmol}, 1$ eq.), compound 73 (651 mg, 1.903 mmol, 1,5 eq.), $n-\mathrm{Bu}_{4} \mathrm{NHSO}_{4}(37 \mathrm{mg}, 0.127 \mathrm{mmol}, 0.1$ eq.) and $2 \mathrm{~mL}$ of solution of $\mathrm{NaOH}$ (50\% aq.) in $\mathrm{CH}_{2} \mathrm{Cl}_{2}(6 \mathrm{~mL})$. An aqueous treatment and chromatography on silica gel (elution with hexane/AcOEt: 80/20) afford $300 \mathrm{mg}$ (41\%) of the product 8 as a yellow oil.

$\mathbf{R f}=0.38$ (hexane/Et $\left.{ }_{2} \mathrm{O}: 50 / 50\right) . \mathbf{N M R}^{1} \mathbf{H}\left(300 \mathrm{MHz}, \mathrm{CDCl}_{3}\right) \delta 7.73(\mathrm{~d}, 2 \mathrm{H}, J=8.1 \mathrm{~Hz}), 7.31$ $(\mathrm{d}, 2 \mathrm{H}, J=7.8 \mathrm{~Hz}), 6.52(\mathrm{t}, 1 \mathrm{H}, J=6.9 \mathrm{~Hz}), 5.84-5.63(\mathrm{~m}, 1 \mathrm{H}), 5.28(\mathrm{~d}, 1 \mathrm{H}, J=17.2 \mathrm{~Hz})$, $5.18(\mathrm{~d}, 1 \mathrm{H}, J=9.7 \mathrm{~Hz}), 4.88(\mathrm{~s}, 1 \mathrm{H}), 4.15-4.10(\mathrm{~m}, 4 \mathrm{H}), 4.06(\mathrm{~s}, 2 \mathrm{H}), 3.82(\mathrm{~d}, 2 \mathrm{H}, J=6.2$ Hz), 2.43 (s, 3H), 2.31-1.97 (m, 4H), 1.90-1.57 (m, 4H), 1.70 (s, 3H), $1.41(\mathrm{~s}, 3 \mathrm{H}) . \mathbf{N M R}{ }^{13} \mathbf{C}$ $\left(75 \mathrm{MHz}, \mathrm{CDCl}_{3}\right) \delta 143.5,136.1,131.9,129.5,127.7,119.9,110.8,88.5,83.9,81.8,80.8$, 79.5, 73.6, 56.4, 56.2, 49.1, 37.5, 36.0, 27.3, 26.3, 24.5, 21.5, 21.5. IR (film): $v=2985,2938$, 2862, 2361, 1598, 1444, 1348, 1255, 1209, 1162, 1081, 1046, 994, 932, 898, 788, 761, 664, $581,546 \mathrm{~cm}^{-1}$.

\section{N-allyl-N-\{[(5bR*,8aS*)-7,7-dimethyl-1,8a-dihydro-3H-5,5b-butano[1,3]dioxolo[3,4]cyclo buta[1,2-e][2]benzofuran-4-yl]methyl\}-4-methylbenzenesulfonamide (16)}

To a solution of compound 8 (100 mg, $0.173 \mathrm{mmol}, 1$ eq. $)$ in benzene $(10 \mathrm{~mL})$ was added tributylstannylethylene (72 mg, $0.225 \mathrm{mmol}, 1.3$ eq.) and $\mathrm{Pd}\left(\mathrm{PPh}_{3}\right)_{4}(20 \mathrm{mg}, 0.017 \mathrm{mmol}, 0.1$ eq.). After $5 \mathrm{~h}$ at $90^{\circ} \mathrm{C}$, the crude mixture was evaporated in vacuo and a chromatography on silica gel (elution with hexane/Et ${ }_{2} \mathrm{O}: 60 / 40$ ), afford $40 \mathrm{mg}$ (47\%) of compound 16 as yellow oil.

$\mathbf{R f}=0.23$ (hexane/Et $\left.{ }_{2} \mathrm{O}: 50 / 50\right) . \mathbf{N M R}^{1} \mathbf{H}\left(300 \mathrm{MHz}, \mathrm{CDCl}_{3}\right) \delta 7.72(\mathrm{~d}, 2 \mathrm{H}, J=7.8 \mathrm{~Hz}), 7.35$ $(\mathrm{d}, 2 \mathrm{H}, J=7.8 \mathrm{~Hz}), 5.47-5.24(\mathrm{~m}, 2 \mathrm{H}), 5.18-4.93(\mathrm{~m}, 4 \mathrm{H}), 4.88(\mathrm{~d}, 1 \mathrm{H}, J=10.3 \mathrm{~Hz}), 4.81(\mathrm{~d}$, $1 \mathrm{H}, J=17.2 \mathrm{~Hz}), 4.37(\mathrm{~d}, 1 \mathrm{H}, J=13.5 \mathrm{~Hz}), 4.15(\mathrm{~d}, 1 \mathrm{H}, J=13.5 \mathrm{~Hz}), 3.55(\mathrm{~d}, 2 \mathrm{H}, J=6.2$ $\mathrm{Hz}), 3.05-2.87(\mathrm{~m}, 1 \mathrm{H}), 2.74-2.57(\mathrm{~m}, 1 \mathrm{H}), 2.46(\mathrm{~s}, 3 \mathrm{H}), 2.31-2.20(\mathrm{~m}, 1 \mathrm{H}), 2.19-1.94(\mathrm{~m}$, 2H), 1.91-1.75 (m, 1H), 1.47 (s, 3H), 1.38-1.19 (m, 2H), $1.02(\mathrm{~s}, 3 \mathrm{H})$. NMR ${ }^{13} \mathbf{C}(75 \mathrm{MHz}$, $\left.\mathrm{CDCl}_{3}\right) \delta 149.0,143.6,142.1,137.7,136.8,135.9,132.6,132.2,129.8,127.4,127.1,118.3$, $115.1,92.5,83.7,73.1,71.4,49.6,46.3,34.7,29.8,29.4,28.8,27.8,27.5,21.5$. IR (film): $v=$ 2928, 2854, 2361, 2251, 1597, 1443, 1346, 1251, 1202, 1161, 1096, 1053, 910, 853, 813, 734, $664,547 \mathrm{~cm}^{-1}$. 


\section{Synthesis of 27 from 87}

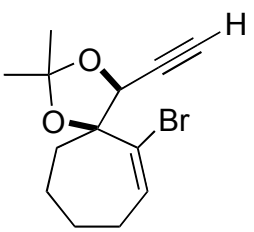

87 7

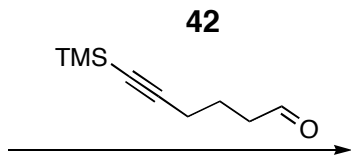

EtMgBr/THF
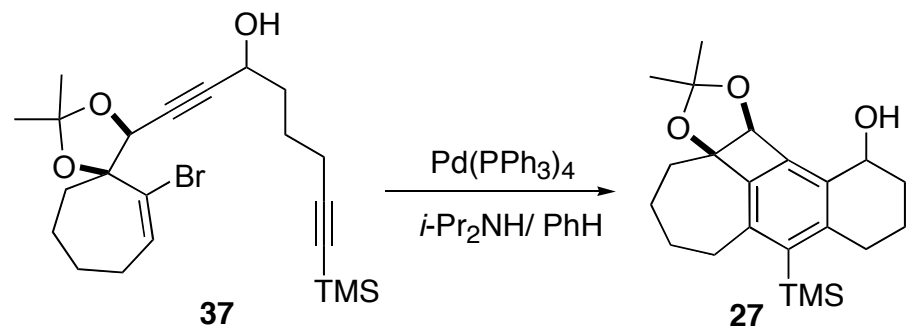

1-[(4S*,5S*)-6-bromo-2,2-dimethyl-1,3-dioxaspiro[4.6] undec-6-en-4-yl]-8-(trimethylsilyl) octa-1,7-diyn-3-ol (37)

To a solution of compound 87 (180 mg, $0.633 \mathrm{mmol}, 1$ eq.) in THF (4 mL) under argon atmosphere at room temperature was added $\mathrm{EtMgBr}(0.7 \mathrm{~mL}, 0.696 \mathrm{mmol}, 1.1$ eq. $)$. After $1 \mathrm{~h}$ at $60^{\circ} \mathrm{C}$, a solution of aldehyde $\mathbf{4 2}^{7}(117 \mathrm{mg}, 0.696 \mathrm{mmol}, 1.1 \mathrm{eq}$.) in THF (1 mL) was added at room temperature. After $18 \mathrm{~h}$ at $40^{\circ} \mathrm{C}$, an aqueous treatment (saturated aqueous solution of $\mathrm{NaHCO}_{3}, 3 * 10 \mathrm{~mL}$ ) and chromatography on silica gel (elution with pentane/Et $\mathrm{Et}_{2} \mathrm{O}: 80 / 20$ ), 192 mg $(67 \%)$ of product 37 was obtained as a yellow oil. The two diastereomers were not separated during the chromatography, ratio: $1 / 1.27 \%$ of the starting material was recovered.

$\mathbf{R f}=0.43\left(\right.$ pentane $\left./ \mathrm{Et}_{2} \mathrm{O}: 70 / 30\right) . \mathbf{N M R}^{1} \mathbf{H}\left(300 \mathrm{MHz}, \mathrm{CDCl}_{3}\right) \delta 6.58(\mathrm{t}, 1 \mathrm{H}, J=6.7 \mathrm{~Hz}), 4.89$ $(\mathrm{m}, 1 \mathrm{H}), 4.49(\mathrm{~m}, 1 \mathrm{H}), 2.28(\mathrm{t}, 2 \mathrm{H}, J=7.0 \mathrm{~Hz}), 2.23-1.89(\mathrm{~m}, 6 \mathrm{H}), 1.88-1.63(\mathrm{~m}, 6 \mathrm{H}), 1.71(\mathrm{~s}$, $3 \mathrm{H}), 1.42(\mathrm{~s}, 3 \mathrm{H}), 0.15(\mathrm{~s}, 9 \mathrm{H}) . \mathbf{N M R}{ }^{13} \mathbf{C}\left(75 \mathrm{MHz}, \mathrm{CDCl}_{3}\right) \delta 136.0,129.4,110.7,110.6$, 106.61, 106.59, 89.43, 89.35, 88.5, 84.6, 79.8, 79.7, 75.3, 61.7, 37.3, 36.2, 27.2, 27.1, 26.3, 24.4, 24.0, 23.9, 21.5, 21.3, 19.4, 0.0. MS (apparatus 3, APPI) : 453.1 [ $\left.\mathrm{M}^{+\bullet}\right], 395.1,265.0$.

\section{$\left(6 \mathrm{~b} R^{*}, 9 \mathrm{a} S^{*}\right)-8,8-$ dimethyl-5-(trimethylsilyl)-1,3,4,9a-tetrahydro-2H-6,6b-butanonaphtho $\left[1^{\prime}, 2^{\prime}: 3,4\right]$ cyclobuta[1,2-d] $[1,3]$ dioxol-1-ol $(27)$}

Compound 27 was prepared following the same procedure than for the preparation of $\mathbf{1 0}$, starting from compound 37 (130 mg, $0.287 \mathrm{mmol}, 1$ eq.), $\mathrm{Pd}\left(\mathrm{PPh}_{3}\right)_{4}(33 \mathrm{mg}, 0,029 \mathrm{mmol}, 0,1$ eq.) and diisopropyl amine $(0.8 \mathrm{~mL})$ in benzene $(2.7 \mathrm{~mL})$. After an aqueous treatment and chromatography on silica gel (elution with pentane/Et $\left.{ }_{2} \mathrm{O}: 80 / 20\right), 39 \mathrm{mg}$ (36\%) of one diastereomer (27a) and $55 \mathrm{mg}(51 \%)$ of the other (27b) as a two yellow solids, the global yield is 88 and the ratio is $4 / 6$.

27a : Rf $=0.29\left(\right.$ pentane/Et $\left._{2} \mathrm{O}: 80 / 20\right) . \mathbf{M p}=128^{\circ} \mathrm{C} . \mathbf{N M R}^{\mathbf{1}} \mathbf{H}\left(300 \mathrm{MHz}, \mathrm{CDCl}_{3}\right) \delta 5.48(\mathrm{~s}$, $1 \mathrm{H}), 4.94(\mathrm{t}, 1 \mathrm{H}, J=5.1 \mathrm{~Hz}), 2.99(\mathrm{dd}, 1 \mathrm{H}, J=5.7 \mathrm{~Hz}, J=15.1 \mathrm{~Hz}), 2.83-2.77(\mathrm{~m}, 3 \mathrm{H}), 2.36$ (br s, 1H), 2.24-1.73 (m, 10H), $1.46(\mathrm{~s}, 3 \mathrm{H}), 1.07(\mathrm{~s}, 3 \mathrm{H}), 0.36(\mathrm{~s}, 9 \mathrm{H}) . \mathbf{N M R}{ }^{13} \mathbf{C}(75 \mathrm{MHz}$, $\left.\mathrm{CDCl}_{3}\right) \delta 146.2,145.2,144.0,143.4,138.5,131.4,114.9,92.2,84.0,66.6,34.9,34.7,32.5$, 31.5, 29.4, 29.2, 28.1, 27.9, 20.0, 4.1. COSY, HSQC, HMBC, NOESY

27b : Rf $=0.13($ pentane/Et $2 \mathrm{O}: 80 / 20) . \mathbf{M p}=118^{\circ} \mathrm{C} . \mathbf{N M R}^{1} \mathbf{H}\left(300 \mathrm{MHz}, \mathrm{CDCl}_{3}\right) \delta 5.38(\mathrm{~s}$, $1 \mathrm{H}), 4.89(\mathrm{t}, 1 \mathrm{H}, J=6.0 \mathrm{~Hz}), 3.06-2.70(\mathrm{~m}, 4 \mathrm{H}), 2.28-1.62(\mathrm{~m}, 11 \mathrm{H}), 1.48(\mathrm{~s}, 3 \mathrm{H}), 1.08(\mathrm{~s}$, $3 \mathrm{H}), 0.38(\mathrm{~s}, 9 \mathrm{H}) . \mathbf{N M R}{ }^{13} \mathbf{C}\left(75 \mathrm{MHz}, \mathrm{CDCl}_{3}\right) \delta 145.7,145.3,144.2,143.0,138.9,132.7$, $115.5,92.5,83.2,66.7,35.0,34.7,32.7,30.7,29.4,29.2,28.0$ (2C), 20.4, 4.1. COSY, HSQC, HMBC, NOESY. GC-MS (apparatus 1) : $372.1\left[\mathrm{M}^{+\bullet}\right], 314.2,296.2,73.1$.

\section{Synthesis of 47 from 43}




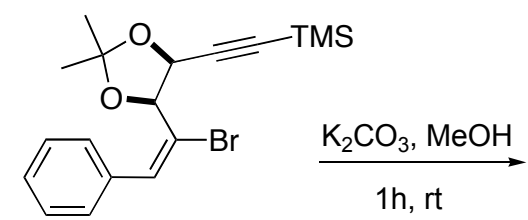

43

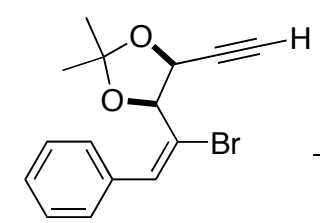

91

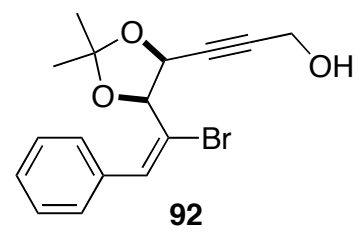

92
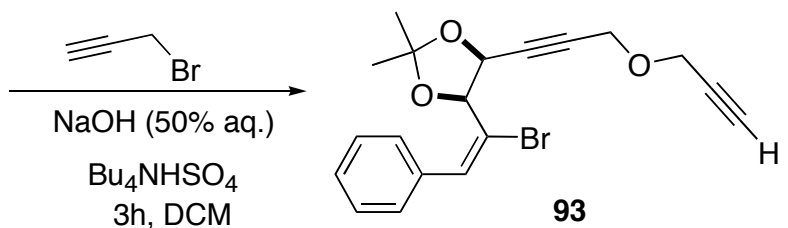

1) BuLi/THF

2) TMSCl

$3 \mathrm{~h}, \mathrm{rt}$

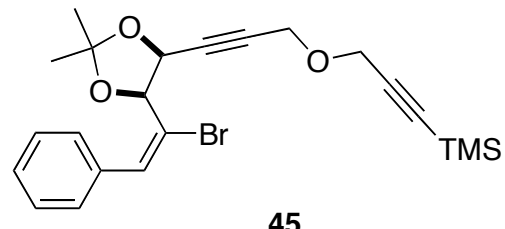

45

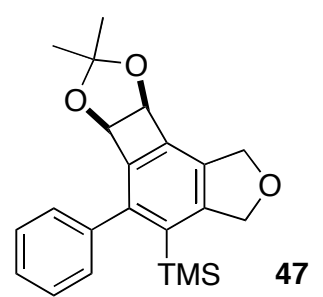

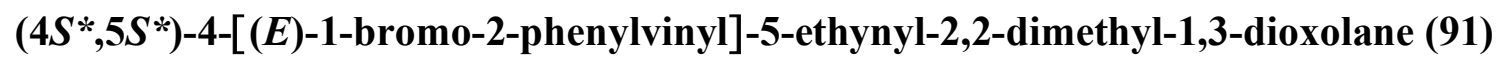

Compound 91 was prepared following the same procedure than for the preparation of 59, starting from compound $\mathbf{4 3}^{8}$ (270 mg, $0.71 \mathrm{mmol}, 1$ eq.) and $\mathrm{K}_{2} \mathrm{CO}_{3}$ (98 $\mathrm{mg}, 0.71 \mathrm{mmol}, 1$ eq.) in $\mathrm{MeOH}(5 \mathrm{~mL})$. After $1 \mathrm{~h}$ of reaction, the same aqueous treatment were realized and the crude product was purified by chromatography on silica gel (elution with heptane/ $\mathrm{Et}_{2} \mathrm{O}$ : $80 / 20)$ to give $218 \mathrm{mg}$ (99\%) of the product 91 as a white powder.

$\mathbf{R f}=0.20\left(\right.$ heptane $\left./ \mathrm{Et}_{2} \mathrm{O}: 95 / 5\right) . \mathbf{N M R}^{1} \mathbf{H}\left(200 \mathrm{MHz}, \mathrm{CDCl}_{3}\right) \delta$ 7.46-7.40 (br s, 5H), $5.03(\mathrm{~s}$, $1 \mathrm{H}, J=5.0 \mathrm{~Hz}), 4.86(\mathrm{dd}, 1 \mathrm{H}, J=5.0 \mathrm{~Hz}, J=2.4 \mathrm{~Hz}), 2.65(\mathrm{~d}, 1 \mathrm{H}, J=2.4 \mathrm{~Hz}), 1.59(\mathrm{~s}, 3 \mathrm{H})$, 1.51 (s, 3H). NMR ${ }^{13} \mathbf{C}\left(50 \mathrm{MHz}, \mathrm{CDCl}_{3}\right) \delta 139.6,135.0,128.5,128.3,124.0,111.6,79.6$, $78.5,76.0,69.4,26.6,26.5$.

\section{$3-\{(4 S *, 5 S *)-5-[(E)-1-b r o m o-2-p h e n y l v i n y l]-2,2-d i m e t h y l-1,3-d i o x o l a n-4-y n y l\}$ prop-2- yn-1-ol (92)}

Compound 92 was prepared following the same procedure than for the preparation of $\mathbf{6 0}$, starting from compound 91 (200 mg, $0.683 \mathrm{mmol}, 1$ eq.), $n$-BuLi (0.55 mL, $0.685 \mathrm{mmol}, 1.05$ eq., $1.24 \mathrm{M}$ in hexane) and paraformaldehyde (30 mg, $0.979 \mathrm{mmol}, 1.5$ eq.) in THF ( $8 \mathrm{~mL})$. After $1 \mathrm{~h}$ at $-78^{\circ} \mathrm{C}$ and $3 \mathrm{~h}$ at RT, an aqueous treatment and a chromatography on silica gel (elution with heptane/Et $\left.\mathrm{t}_{2} \mathrm{O}: 80 / 20\right), 180 \mathrm{mg}(81 \%)$ of product 92 was obtained as a colourless oil.

$\mathbf{R f}=0.25$ (heptane/Et $\left.\mathrm{Et}_{2}: 70 / 30\right) . \mathbf{N M R}{ }^{1} \mathbf{H}\left(200 \mathrm{MHz}, \mathrm{CDCl}_{3}\right) \delta 7.38-7.35(\mathrm{~m}, 4 \mathrm{H}), 7.17(\mathrm{~d}$, $2 \mathrm{H}, J=7.3 \mathrm{~Hz}), 5.09$ (br s, 2H), $4.35(\mathrm{~s}, 2 \mathrm{H}), 2.60(\mathrm{~s}, 1 \mathrm{H}), 1.68(\mathrm{~s}, 3 \mathrm{H}), 1.32(\mathrm{~s}, 3 \mathrm{H}) . \mathbf{N M R}$ ${ }^{13} \mathbf{C}\left(50 \mathrm{MHz}, \mathrm{CDCl}_{3}\right) \delta 139.7,134.9,128.5,128.2,128.2,124.0,111.6,78.5,77.3,76.0$, $69.4,62.5,25.9,25.8$.

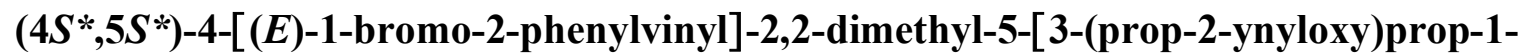
ynyl]-1,3-dioxolane (93) 
Compound 93 was prepared following the same procedure than for the preparation of $\mathbf{6 1}$, starting from compound 92 (180 $\mathrm{mg}, 0.53 \mathrm{mmol}, 1$ eq. $)$, propargyl bromine $(0.154 \mathrm{mg}, 1.7$ mmol, 3.2 eq., $80 \%$ in toluene), $n \mathrm{Bu}_{4} \mathrm{NHSO}_{4}(11 \mathrm{mg}, 0.05 \mathrm{mmol}, 0.1$ eq.) and $1 \mathrm{~mL}$ of solution of $\mathrm{NaOH}\left(50 \%\right.$ aq.) in $\mathrm{CH}_{2} \mathrm{Cl}_{2}(3 \mathrm{~mL})$. Aqueous treatment and chromatography on silica gel (elution with heptane/ $\left.\mathrm{Et}_{2} \mathrm{O}: 80 / 20\right)$ afford $140 \mathrm{mg}(70 \%)$ of the product 93 as a yellow oil.

$\mathbf{R f}=0.67\left(\right.$ heptane/Et $\left.{ }_{2} \mathrm{O}: 60 / 40\right) . \mathbf{N M R}^{1} \mathbf{H}\left(200 \mathrm{MHz}, \mathrm{CDCl}_{3}\right) \delta 7.38-7.30(\mathrm{~m}, 4 \mathrm{H}), 7.16(\mathrm{~d}$, $2 \mathrm{H}, J=6.9 \mathrm{~Hz}), 5.09(\mathrm{~d}, 1 \mathrm{H}, J=7.6 \mathrm{~Hz}), 5.06(\mathrm{~d}, 1 \mathrm{H}, J=7.6 \mathrm{~Hz}), 4.35(\mathrm{~s}, 2 \mathrm{H}), 4.29(\mathrm{~d}, 2 \mathrm{H}$, $J=2.3 \mathrm{~Hz}), 2.39(\mathrm{~d}, 1 \mathrm{H}, J=2.3 \mathrm{~Hz}), 1.67(\mathrm{~s}, 3 \mathrm{H}), 1.30(\mathrm{~s}, 3 \mathrm{H}) . \mathbf{N M R}{ }^{13} \mathbf{C}\left(50 \mathrm{MHz}, \mathrm{CDCl}_{3}\right)$ $\delta 136.8,135.9,128.7,128.6,128.2,126.5,111.2,84.6,81.0,79.1,75.9,75.2,69.7,57.6$, 57.0, 26.2, 25.1. MS (apparatus 5, positif ion) $\left(\mathrm{C}_{19} \mathrm{H}_{19} \mathrm{O}_{3} \mathrm{Na}\right)^{+}$calc. : 398.25, found : 398.27.

\section{$\left\{3-\left[\left(3-\left\{\left(4 S^{*}, 5 S^{*}\right)-5-[(Z)-1-b r o m o-2-p h e n y l v i n y l]-2,2-d i m e t h y l-1,3-d i o x o l a n-4-y l\right\}\right.\right.\right.$ prop-2- ynyl)oxy]prop-1-ynyl $\}$ (trimethyl) silane (45)}

Compound 45 was prepared following the same procedure than for the preparation of $\mathbf{1}$, starting from compound $93(120 \mathrm{mg}, 0.320 \mathrm{mmol}, 1$ eq. $), n$-BuLi $(0.22 \mathrm{~mL}, 0.33 \mathrm{mmol}, 1.05$ eq.) and $\mathrm{TMSCl}(38 \mathrm{mg}, 0.35 \mathrm{mmol}, 1.1$ eq.) in anhydrous THF (7 mL). After an aqueous treatment and chromatography on silica gel (elution with heptane/Et $\left.{ }_{2} \mathrm{O}: 80 / 20\right), 101 \mathrm{mg}(71 \%)$ of product 45 was obtained as a brown oil.

$\mathbf{R f}=0.67($ heptane/Et $2 \mathrm{O}: 60 / 40) . \mathbf{N M R}{ }^{1} \mathbf{H}\left(200 \mathrm{MHz}, \mathrm{CDCl}_{3}\right) \delta 7.38-7.35(\mathrm{~m}, 4 \mathrm{H}), 7.19(\mathrm{~d}$, $2 \mathrm{H}, J=7.6 \mathrm{~Hz}), 5.11(\mathrm{~d}, 1 \mathrm{H}, J=7.4 \mathrm{~Hz}), 5.09(\mathrm{~d}, 1 \mathrm{H}, J=7.4 \mathrm{~Hz}), 4.36(\mathrm{~s}, 2 \mathrm{H}), 4.30(\mathrm{~s}, 2 \mathrm{H})$, $1.69(\mathrm{~s}, 3 \mathrm{H}), 1.33(\mathrm{~s}, 3 \mathrm{H}), 0.16(\mathrm{~s}, 9 \mathrm{H}) . \mathbf{N M R}{ }^{13} \mathbf{C}\left(50 \mathrm{MHz}, \mathrm{CDCl}_{3}\right) \delta 137.0,136.3,129.0$, $128.8,128.4,126.7,111.5,101.0,92.4,84.9,81.2,76.2,70.0,57.6,57.0,25.5,25.4,0.2$. IR (FITR, film): $v=2957,2925,2852,2079,1623,1493,1380,1250,1161,1000,921,877$, $843,754,700 \mathrm{~cm}^{-1}$.

\section{$\left[\left(5 \mathrm{~b} R *, 8 \mathrm{a} S^{*}\right)-7,7-d i m e t h y l-5-p h e n y l-1,35 \mathrm{~b}, 8 \mathrm{a}-\right.$}

tetrahydro[ $[1,3]$ dioxolo $[3,4]$ cyclobutan[ $[1,2-e][2]$ benzofuran-4-yl] (trimethylsilane) (47)

Compound 47 was prepared following the same procedure than for the preparation of $\mathbf{1 0}$, starting from compound 45 (20 mg, $0.05 \mathrm{mmol}, 1$ eq.), $\mathrm{Pd}\left(\mathrm{PPh}_{3}\right)_{4}$ (7 mg, $0.005 \mathrm{mmol}, 0.1$ eq.) and diisopropyl amine $(0.2 \mathrm{~mL})$ in benzene $(2 \mathrm{~mL})$. After an aqueous treatment and chromatography on silica gel (elution with heptane/Et ${ }_{2} \mathrm{O}$ : 90/10), $12 \mathrm{mg}$ (65\%) of product 47 was obtained as a yellow oil.

$\mathbf{R f}=0.32\left(\right.$ heptane/Et $\left.{ }_{2} \mathrm{O}: 80 / 20\right) . \mathbf{N M R}^{1} \mathbf{H}\left(300 \mathrm{MHz}, \mathrm{CDCl}_{3}\right) \delta$ 7.45-7.32 (m, 3H), $7.25(\mathrm{~d}$, $2 \mathrm{H}, J=7.8 \mathrm{~Hz}), 5.27(\mathrm{~s}, 1 \mathrm{H}, J=6.3 \mathrm{~Hz}), 5.15-5.03(\mathrm{~m}, 3 \mathrm{H}), 4.72(\mathrm{~s}, 2 \mathrm{H}, J=17.3 \mathrm{~Hz}), 1.51$ $(\mathrm{s}, 3 \mathrm{H}), 1.41(\mathrm{~s}, 3 \mathrm{H}), 0.33 . \mathbf{N M R}{ }^{13} \mathbf{C}\left(50 \mathrm{MHz}, \mathrm{CDCl}_{3}\right) \delta 149.5,146.8,140.5,139.7,139.6$, 135.2, $134.0,133.1,132.3,131.0,114.3,83.3,81.2,74.2,70.5,29.0,28.2$, 1.0. IR (FITR, film): $v=3372,2944,2850,1618,1435,1379,1305,1256,1199,1147,1095,1062 \mathrm{~cm}^{-1}$.

COSY, HSQC, HMBC, NOESY.

\section{Synthesis of 46 from 44}



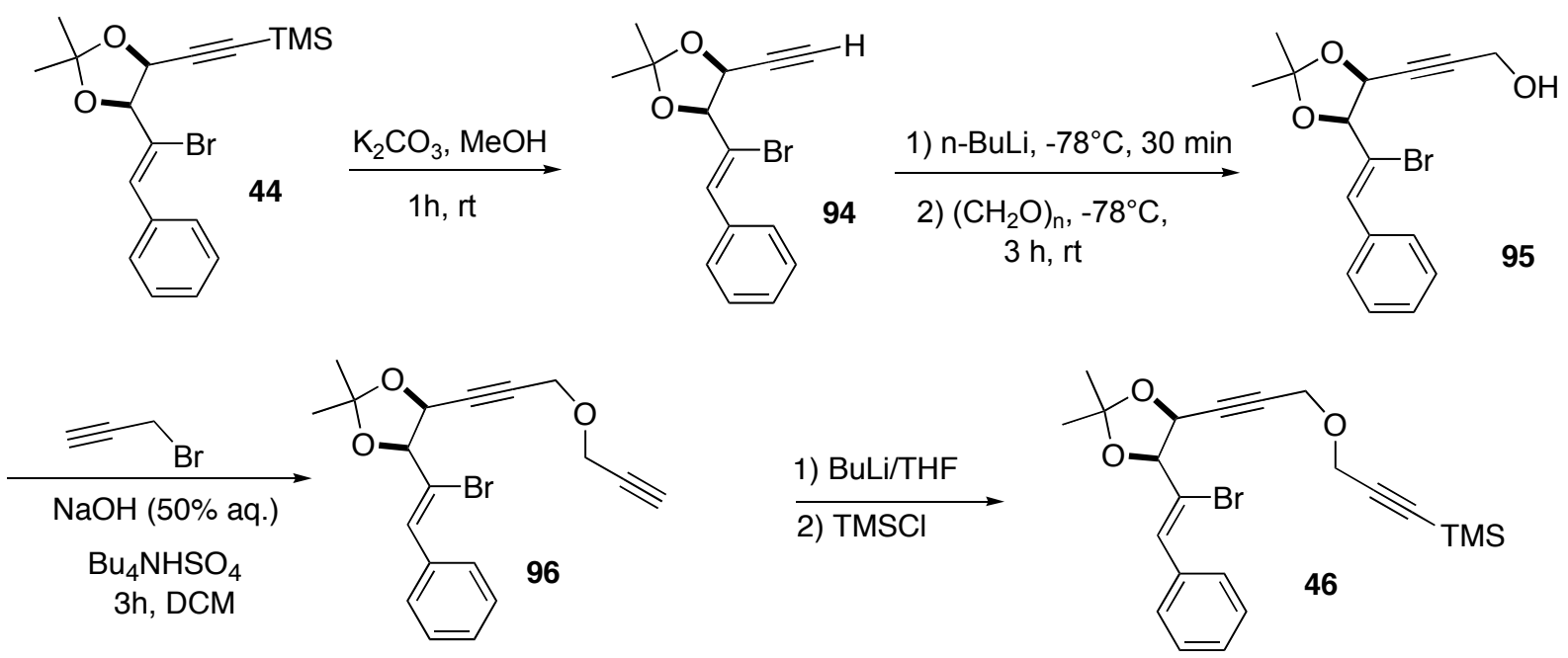

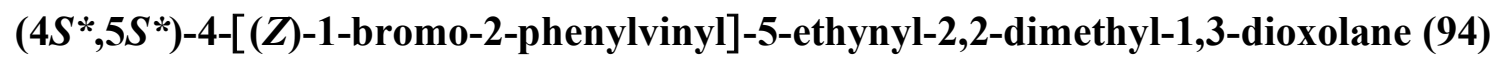

Compound 94 was prepared following the same procedure than for the preparation of 59, starting from compound $\mathbf{4 4}^{8}$ (270 mg, $0.71 \mathrm{mmol}, 1$ eq.) and $\mathrm{K}_{2} \mathrm{CO}_{3}(98 \mathrm{mg}, 0.71 \mathrm{mmol}, 1$ eq.) in $\mathrm{MeOH}(5 \mathrm{~mL})$. After $1 \mathrm{~h}$ of reaction, the same aquous treatment was applied and the crude product was purified by chromatography on silica gel (elution with heptane/Et ${ }_{2} \mathrm{O}: 95 / 5$ ) to give $187 \mathrm{mg}(86 \%)$ of the product 94 as a white powder.

Rf $=0.33$ (heptane/Et $\left.{ }_{2} \mathrm{O}: 95 / 5\right) .{ }^{1} \mathbf{H}$ NMR $\left(200 \mathrm{MHz}, \mathrm{CDCl}_{3}\right) \delta 7.42-7.38$ (br s, 5H), 4.75 (s, $1 \mathrm{H}, J=5.5 \mathrm{~Hz}), 4.62(\mathrm{dd}, 1 \mathrm{H}, J=5.5 \mathrm{~Hz}, J=3.2 \mathrm{~Hz}), 2.65(\mathrm{~d}, 1 \mathrm{H}, J=3.2 \mathrm{~Hz}), 1.55(\mathrm{~s}, 3 \mathrm{H})$, $1.45(\mathrm{~s}, 3 \mathrm{H}) .{ }^{13} \mathbf{C}$ NMR $\left(50 \mathrm{MHz}, \mathrm{CDCl}_{3}\right) \delta 136.6,135.8,128.5,128.4,126.1,111.2,77.7$, 76.6, 75.7, 69.3, 26.0, 24.9.

\section{3-\{(4S*,5S*)-5-[(Z)-1-bromo-2-phenylvinyl]-2,2-dimethyl-1,3-dioxolan-4-ynyl $\}$ prop-2- yn-1-ol (95)}

Compound 95 was prepared following the same procedure than for the preparation of 60 , starting from compound 94 (200 mg, $0.683 \mathrm{mmol}, 1$ eq.), $n$-BuLi $(0.55 \mathrm{~mL}, 0.685 \mathrm{mmol}, 1.05$ eq., $1.24 \mathrm{M}$ in hexane) and paraformaldehyde (30 mg, $0.979 \mathrm{mmol}, 1.5$ eq.) in THF (10 mL). After $1 \mathrm{~h}$ at $-78^{\circ} \mathrm{C}$ and $3 \mathrm{~h}$ at $\mathrm{RT}$, an aqueous treatment and chromatography on silica gel (elution with hexane/Et $\left.{ }_{2} \mathrm{O}: 80 / 20\right), 120 \mathrm{mg}$ (54\%) of product 95 was obtained as a colourless oil.

$\mathbf{R f}=0.32\left(\right.$ heptane $\left./ \mathrm{Et}_{2} \mathrm{O}: 70 / 30\right) .{ }^{1} \mathbf{H}$ NMR $\left(200 \mathrm{MHz}, \mathrm{CDCl}_{3}\right) \delta$ 7.45-7.34 (m, 4H), $7.25(\mathrm{~d}$, $2 \mathrm{H}, J=7.1 \mathrm{~Hz}), 5.13(\mathrm{~s}, 2 \mathrm{H}), 4.32(\mathrm{~s}, 2 \mathrm{H}), 2.05(\mathrm{se}, 1 \mathrm{H}), 1.69(\mathrm{~s}, 3 \mathrm{H}), 1.32(\mathrm{~s}, 3 \mathrm{H}) .{ }^{13} \mathrm{C} \mathrm{NMR}$ $\left(50 \mathrm{MHz}, \mathrm{CDCl}_{3}\right) \delta 139.6,134.9,128.5,128.3,128.1,124.0,111.6,79.2,78.2,74.1,69.0$, $62.8,26.4,26.0$.

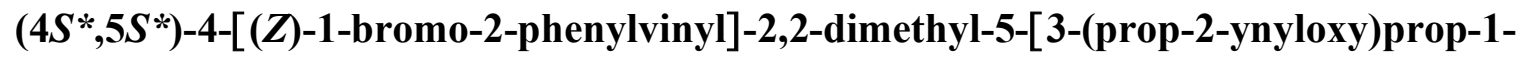
ynyl]-1,3-dioxolane (96)

Compound 96 was prepared following the same procedure than for the preparation of $\mathbf{6 1}$, starting from compound 95 (120 mg, $0.53 \mathrm{mmol}, 1 \mathrm{eq}$.$) , propargyl bromine (0.154 \mathrm{mg}, 1.02$ mmol, 3.2 eq., $80 \%$ in toluene), $n \mathrm{Bu}_{4} \mathrm{NHSO}_{4}(11 \mathrm{mg}, 0.03 \mathrm{mmol}, 0.1$ eq.) and $1 \mathrm{~mL}$ of solution of $\mathrm{NaOH}\left(50 \%\right.$ aq.) in $\mathrm{CH}_{2} \mathrm{Cl}_{2}(3 \mathrm{~mL})$. Aqueous treatment and chromatography on silica gel (elution with hexane/ $\mathrm{Et}_{2} \mathrm{O}: 80 / 20$ ) afford $98 \mathrm{mg}(82 \%)$ of the product 96 as a yellow oil. 
$\mathbf{R f}=0.55($ heptane/Et $2 \mathrm{O}: 60 / 40) .{ }^{1} \mathbf{H}$ NMR $\left(200 \mathrm{MHz}, \mathrm{CDCl}_{3}\right) \delta$ 7.34-7.28 (m, 4H), $7.12(\mathrm{~d}$, $2 \mathrm{H}, J=6.0 \mathrm{~Hz}), 5.12(\mathrm{~d}, 1 \mathrm{H}, J=7.4 \mathrm{~Hz}), 5.04(\mathrm{~d}, 1 \mathrm{H}, J=7.4 \mathrm{~Hz}), 4.32(\mathrm{~s}, 2 \mathrm{H}), 4.28(\mathrm{~d}, 2 \mathrm{H}$, $J=2.2 \mathrm{~Hz}), 2.39(\mathrm{~d}, 1 \mathrm{H}, J=2.2 \mathrm{~Hz}), 1.59$ (s, 3H), $1.32(\mathrm{~s}, 3 \mathrm{H}) .{ }^{13} \mathbf{C}$ NMR $\left(50 \mathrm{MHz}, \mathrm{CDCl}_{3}\right)$ $\delta$ 136.7, 135.8, 128.8, 128.6, 128.2, 126.5, 111.2, 84.6, 81.0, 79.2, 76.2, 75.2, 69.8, 57.6, 57.0, 26.2, 25.0. IR (FITR, film): $v=3286,2986,2940,2861,2118,1633,1447,1378$, $1255,1211,1164,1122,990,965,901,806,710,669 \mathrm{~cm}^{-1}$.

\section{\{3-[(3- $\left\{\left(4 S^{*}, 5 S^{*}\right)-5-[(E)-1-b r o m o-2-p h e n y l v i n y l]-2,2-d i m e t h y l-1,3-d i o x o l a n-4-y l\right\}$ prop-2- ynyl)oxy]prop-1-ynyl\}(trimethyl) silane (46)}

Compound 46 was prepared following the same procedure than for the preparation of $\mathbf{1}$, starting from compound 96 (120 mg, $0.320 \mathrm{mmol}, 1$ eq. $), n$-BuLi $(0.22 \mathrm{~mL}, 0.33 \mathrm{mmol}, 1.05$ eq.) and $\mathrm{TMSCl}(38 \mathrm{mg}, 0.35 \mathrm{mmol}, 1.1$ eq.) in anhydrous THF (5 mL). After an aqueous treatment and chromatography on silica gel (elution with heptane/Et $\left.{ }_{2} \mathrm{O}: 80: 20\right), 60 \mathrm{mg}(42 \%)$ of product 46 was obtained as a brown oil.

$\mathbf{R f}=0.45($ heptane/Et $2 \mathrm{O}: 80 / 20) .{ }^{1} \mathbf{H}$ NMR $\left(200 \mathrm{MHz}, \mathrm{CDCl}_{3}\right) \delta 7.45-7.32(\mathrm{~m}, 4 \mathrm{H}), 7.15(\mathrm{~d}$, $2 \mathrm{H}, J=7.2 \mathrm{~Hz}), 5.18(\mathrm{~d}, 1 \mathrm{H}, J=7.2 \mathrm{~Hz}), 5.11(\mathrm{~d}, 1 \mathrm{H}, J=7.2 \mathrm{~Hz}), 4.31(\mathrm{~s}, 2 \mathrm{H}), 4.25(\mathrm{~s}, 2 \mathrm{H})$, $1.67(\mathrm{~s}, 3 \mathrm{H}), 1.35(\mathrm{~s}, 3 \mathrm{H}), 0.25(\mathrm{~s}, 9 \mathrm{H}) .{ }^{13} \mathbf{C ~ N M R}\left(50 \mathrm{MHz}, \mathrm{CDCl}_{3}\right) \delta 139.4,136.8,128.7$, $128.7,128.3,127.0,112.0,101.0,92.6,84.2,81.3,76.2,71.0,57.7,56.6,27.3,26.5,0.1$.

\section{Synthesis of 56 from 48}

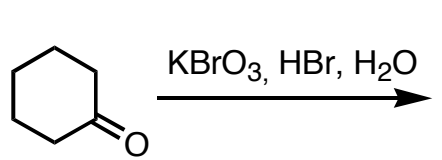

48<smiles>O=C1CCCC(=O)C1Br</smiles>

97

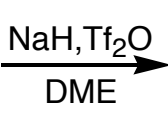

$\mathrm{ME}$<smiles>O=C1CCCC(OOC(F)(F)F)=C1Br</smiles>

98

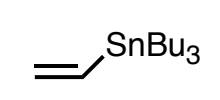

$\mathrm{Pd}(\mathrm{OAc})_{2}, \mathrm{PPh}_{3}$

THF

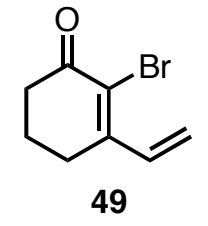

49

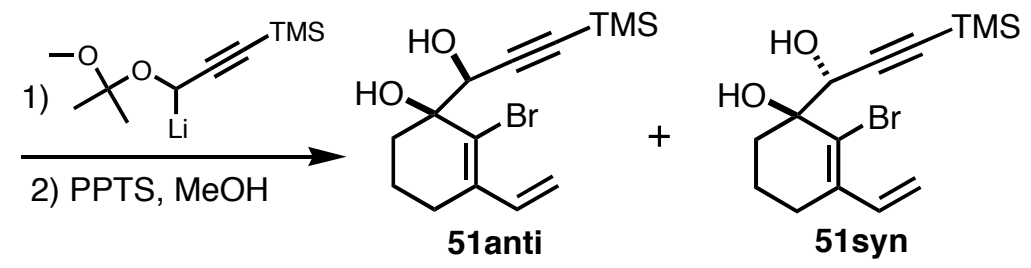

$\left.\downarrow \begin{array}{c}\mathrm{Pd}\left(\mathrm{PPh}_{3}\right)_{4} \\ i-\mathrm{Pr}_{2} \mathrm{NH} / \mathrm{PhH}\end{array}\right\rfloor$<smiles>Cc1ccc2c(c1CO)C(=O)CCC2</smiles>

54

\section{2-Bromo-1,3-cyclohexanedione (97)}

To a solution of cyclohexane-1,3-dione 48 (11.2 g, $0.1 \mathrm{~mol}, 1$ eq.) and hydrobromic acid (15 $\mathrm{mL}, 0.1 \mathrm{~mol}, 1 \mathrm{eq}$. $)$ in water $(50 \mathrm{~mL})$ was added slowly a solution of potasium bromate $(5.7 \mathrm{~g}$, $0.033 \mathrm{~mol}, 1$ eq.) in water $(50 \mathrm{~mL})$ at $40^{\circ} \mathrm{C}$. At the end of the addition, the precipitate was filtrated and washed with water $(3 * 50 \mathrm{~mL})$, concentrated and dried during $3 \mathrm{~h}(12 \mathrm{mbar}$, $\left.50^{\circ} \mathrm{C}\right) .15 .79 \mathrm{~g}(82 \%)$ of product 97 was obtained as a beige solid. 
$\mathbf{M p}=85^{\circ} \mathrm{C},{ }^{1} \mathbf{H}$ NMR $\left(200 \mathrm{MHz}, \mathrm{CDCl}_{3}\right) \delta 5.54(\mathrm{se}, 1 \mathrm{H}), 2.60(\mathrm{t}, 4 \mathrm{H}, J=6.7 \mathrm{~Hz}), 2.01(\mathrm{q}$, $2 \mathrm{H}, J=12.0 \mathrm{~Hz}) .{ }^{13} \mathbf{C} \mathbf{N M R}\left(75 \mathrm{MHz}, \mathrm{CDCl}_{3}\right) \delta 193.5,97.0,30.9,26.0$.

\section{2-bromo-3-oxocyclohex-1-en-1-yl trifluoromethanesulfonate (98)}

To a suspension of $\mathrm{NaH}(760 \mathrm{mg}, 19 \mathrm{mmol}, 1.05$ eq., $60 \%$ in oil) in anhydrous dimethoxyether $(80 \mathrm{~mL})$ at $0^{\circ} \mathrm{C}$ was added slowly compound 97 (3.82 g, $20 \mathrm{mmol}, 1.1 \mathrm{eq}$ ). After $20 \mathrm{~min}$, the reaction mixture was warmed to room temperature during $2 \mathrm{~h}$. Then at $78^{\circ} \mathrm{C}$, triflic anhydride $(5.2 \mathrm{~g}, 18.4 \mathrm{mmol}, 1 \mathrm{eq}$.$) was added slowly in 2 \mathrm{~h}$. After addition, the reaction mixture was stirred at room temperature during $20 \mathrm{~min}$ and concentrated. The reaction mixture was extracted with $\mathrm{CH}_{2} \mathrm{Cl}_{2}(100 \mathrm{~mL})$ and the combined organic layers were washed with brine, dried over $\mathrm{Na}_{2} \mathrm{SO}_{4}$ and concentrated in vacuo. The crude product was purified by chromatography on silica gel (elution with $\mathrm{CH}_{2} \mathrm{Cl}_{2}$ ) to afford $3.98 \mathrm{~g}(61 \%)$ of the product 98 as a viscous oil stocked at $-20^{\circ} \mathrm{C}$.

Rf : $0.63\left(\mathrm{CHCl}_{3}\right) .{ }^{1} \mathbf{H}$ NMR $\left(200 \mathrm{MHz}, \mathrm{CDCl}_{3}\right) \delta 2.96(\mathrm{t}, 2 \mathrm{H}, J=6.2 \mathrm{~Hz}), 2.68(\mathrm{t}, 2 \mathrm{H}, J=$ $6.2 \mathrm{~Hz}), 2,01(\mathrm{q}, 2 \mathrm{H}, J=6.3 \mathrm{~Hz}) .{ }^{13} \mathbf{C}$ NMR $\left(75 \mathrm{MHz}, \mathrm{CDCl}_{3}\right) \delta 190.5,164.2,120.7(\mathrm{t}, J=$ $317.3 \mathrm{~Hz}$ ), 117.8, 37.1, 30.8, 20.7. MS (apparatus 5) : 324, $322[M]^{+}, 174,172[M-$ $\left.\mathrm{CF}_{3} \mathrm{SO}_{3} \mathrm{H}\right]^{+}, 69\left[\mathrm{CF}_{3}\right]^{+}$. Microanalysis : $\mathrm{C}_{7} \mathrm{H}_{6} \mathrm{BrF}_{3} \mathrm{O}_{4} \mathrm{~S}$ : calcd : $\mathrm{C}(26.02), \mathrm{H}(1.87)$. Found : $\mathrm{C}(25.89), \mathrm{H}(1.77)$.

\section{2-bromo-3-ethenyl- 2-cyclohexen-1-one (49)}

A mixture of compound 98 (1.96 g, $6.06 \mathrm{mmol}, 1$ eq.), vinyltributylstannane $(2.3 \mathrm{~g}, 7.26$ mmol, 1.2 eq.), triphenylphosphine ( $222 \mathrm{mg}, 0.84 \mathrm{mmol}, 0.1$ eq.), and $\mathrm{Pd}(\mathrm{OAc})_{2}$ (96 mg, 0.42 mmol, 0,05 eq.) in anhydrous THF $(100 \mathrm{~mL})$ were stired during $22 \mathrm{~h}$ at room temperature and concentrated in vacuo. The crude product was purified by chromatography on silica gel (elution with AcOEt/Heptane: 40/60) to afford $1.11 \mathrm{~g} \mathrm{(91 \% )} \mathrm{of} \mathrm{the} \mathrm{product} 49$ as a beige solid stored at $-20^{\circ} \mathrm{C}$.

Rf : 0.30 (heptane/AcOEt: 60/40). ${ }^{1} \mathbf{H}$ NMR $\left(200 \mathrm{MHz}, \mathrm{CDCl}_{3}\right) \delta 7.14(\mathrm{dd}, 1 \mathrm{H}, J=10.2 \mathrm{~Hz}$, $J=17.6 \mathrm{~Hz}), 5.79(\mathrm{~d}, 1 \mathrm{H}, J=17.6 \mathrm{~Hz}), 5.63(\mathrm{~d}, 1 \mathrm{H}, J=10.2 \mathrm{~Hz}), 2.67-2.59(\mathrm{~m}, 4 \mathrm{H}), 2.04(\mathrm{q}$, $2 \mathrm{H}, J=6,3 \mathrm{~Hz}) .{ }^{13} \mathbf{C}$ NMR $\left(75 \mathrm{MHz}, \mathrm{CDCl}_{3}\right) \delta 191.8,153.1,136.9,124.3,123.8,38.1,27.3$, 21.4. MS (apparatus 5) $\left(\mathrm{C}_{8} \mathrm{H}_{9} \mathrm{BrONa}\right)^{+}$calc.: 224.05 , found: 224.03 .

(1S*)-2-bromo-1-[(1S*)-1-hydroxy-3-(trimethylsilyl)prop-2-ynyl]-3-vinylcyclohex-2-en1-ol (51anti) and $\left(1 S^{*}\right)$-2-bromo-1-[(1R*)-1-hydroxy-3-(trimethylsilyl)prop-2-ynyl]-3vinylcyclohex-2-en-1-ol (51syn)

A solution of $n$-butyllithium (4.12 mL, $6.30 \mathrm{mmol}, 1.41$ eq., $1.53 \mathrm{M}$ in hexane) was added to a dried round-bottom flask containing 3-(1-methoxy-1-methyl-ethoxy)-prop-1-ynyl]trimethylsilyl ${ }^{9}$ (1.25 g, $6.25 \mathrm{mmol}, 1.4$ eq. $)$ in freshly distilled THF $(5 \mathrm{~mL})$ at $-78{ }^{\circ} \mathrm{C}$. The reaction was stirred $30 \mathrm{~min}$ at this temperature and a solution of 49 (900 $\mathrm{mg}, 4.47 \mathrm{mmol}$, 1eq.) in THF $(7 \mathrm{~mL})$ was then added via cannula. The mixture was stirred for $2 \mathrm{~h}$ at $-78{ }^{\circ} \mathrm{C}$ and then quenched by addition of saturated aqueous $\mathrm{NaHCO}_{3}(50 \mathrm{~mL})$ and water $(100 \mathrm{~mL})$. After extraction with $\mathrm{Et}_{2} \mathrm{O}$, the combined organic layers were washed with water, brine, dried over $\mathrm{Na}_{2} \mathrm{SO}_{4}$ and concentrated in vacuo. The crude mixture were dissolved in methanol $(60 \mathrm{~mL})$ containing catalytique quantity of PPTS ( 0.1 eq.). The solution was stirred for $1 \mathrm{~h}$ at $\mathrm{rt}$ and

9 Suffert, J.; Salem, B.; Klotz, P. J. Am. Chem. Soc. 2001, 123, 12107 
quenched with the addition of brine and water. The mixture was extracted with $\mathrm{Et}_{2} \mathrm{O}$ and the combined extracts were washed with water, brine and dried over $\mathrm{Na}_{2} \mathrm{SO}_{4}$. The two diastereomeres were separated by chromatography on silica gel (heptane/ $\left.\mathrm{Et}_{2} \mathrm{O}: 95 / 5\right)$ to afford compounds 51syn (280 mg, 27\%) and 51anti (730 mg, 41) as white solid for a global yield of $68 \%$ and a ratio of $2 / 3$.

51anti : $\mathbf{R f}=0.39\left(\right.$ heptane/Et $\left.{ }_{2} \mathrm{O}: 50 / 50\right) .{ }^{1} \mathbf{H}$ NMR $\left(200 \mathrm{MHz}, \mathrm{CDCl}_{3}\right) \delta 6.91(\mathrm{dd}, 1 \mathrm{H}, J=$ $11.2 \mathrm{~Hz}, J=17.2 \mathrm{~Hz}), 5.41(\mathrm{~d}, 1 \mathrm{H}, J=17.2 \mathrm{~Hz}), 5.29(\mathrm{~d}, 1 \mathrm{H}, J=11.2 \mathrm{~Hz}), 4.71(\mathrm{~d}, 1 \mathrm{H}, J=$ $6.0 \mathrm{~Hz}), 2.59(\mathrm{~s}, 1 \mathrm{H}), 2.49(\mathrm{td}, 1 \mathrm{H}, J=16.8 \mathrm{~Hz}, J=3.2 \mathrm{~Hz}), 2.20(\mathrm{~d}, 1 \mathrm{H}, J=6 \mathrm{~Hz}), 2.17-2.01$ $(\mathrm{m}, 3 \mathrm{H}), 1.90-1.71(\mathrm{~m}, 3 \mathrm{H}), 0.18(\mathrm{~s}, 9 \mathrm{H}) .{ }^{3} \mathbf{C ~ N M R}^{1}$ (75 MHz, $\left.\mathrm{CDCl}_{3}\right) \delta 139.1,137.0,127.8$, 118.1, 102.7, 92.6, 75.3, 69.4, 32.9, 27.8, 17.9, -0.3. MS (apparatus 5) : $\left(\mathrm{C}_{14} \mathrm{H}_{21} \mathrm{BrO}_{2} \mathrm{SiNa}\right)^{+}$ calc. : 351.04 , found : 351.05 .

51syn : Rf $=0.33\left(\right.$ heptane/Et $\left.\mathrm{O}_{2}: 50 / 50\right) . \mathbf{N M R}{ }^{1} \mathbf{H}\left(200 \mathrm{MHz}, \mathrm{CDCl}_{3}\right) \delta 6.93(\mathrm{dd}, 1 \mathrm{H}, J=$ $10.9 \mathrm{~Hz}, J=18.4 \mathrm{~Hz}), 5.40(\mathrm{~d}, 1 \mathrm{H}, J=18.4 \mathrm{~Hz}), 5.28(\mathrm{~d}, 1 \mathrm{H}, J=10.9 \mathrm{~Hz}), 4.70(\mathrm{br} \mathrm{s}, 1 \mathrm{H})$, $2.61(\mathrm{~s}, 1 \mathrm{H}), 2.41(\mathrm{td}, 1 \mathrm{H}, J=16.3 \mathrm{~Hz}, J=2.8 \mathrm{~Hz}), 2.28-2.05(\mathrm{~m}, 4 \mathrm{H}), 1.90-1.63(\mathrm{~m}, 2 \mathrm{H})$, $0.17(\mathrm{~s}, 9 \mathrm{H}) . \mathbf{N M R}{ }^{13} \mathbf{C}\left(75 \mathrm{MHz}, \mathrm{CDCl}_{3}\right) \delta 139.2,137.0,127.8,118.1,102.7,92.7,75.5$, 69.6, 32.9, 27.8, 17.9, -0.3. MS (apparatus 5): $\left(\mathrm{C}_{14} \mathrm{H}_{21} \mathrm{BrO}_{2} \mathrm{SiNa}\right)^{+}$calc. : 351.03, found : 349.99 .

\section{8-(hydroxymethyl)-7-(trimethylsilyl)-3,4-dihydronaphtalen-1(2H)-one (56)}

Compound 54 was prepared following the same procedure than for the preparation of $\mathbf{1 0}$, starting from compound $\mathbf{5 1 \text { anti }}(23 \mathrm{mg}, 0.07 \mathrm{mmol}$, 1 eq.) or $\mathbf{5 1 s y n}(23 \mathrm{mg}, 0.07 \mathrm{mmol}, 1 \mathrm{eq}$.$) ,$ $\mathrm{Pd}\left(\mathrm{PPh}_{3}\right)_{4}(8 \mathrm{mg}, 0.007 \mathrm{mmol}, 0.1$ eq. $)$ and diisopropyl amine $(0.2 \mathrm{~mL})$ in benzene $(0.6 \mathrm{~mL})$. After chromatography on silica gel (elution with heptane/Et $\left.{ }_{2} \mathrm{O}: 90 / 10\right), 12 \mathrm{mg}(70 \%)$ of product 54 from $\mathbf{5 1 a n t i}$ and $10 \mathrm{mg}(64 \%)$ from $\mathbf{5 1 s y n}$ were obtained as a white solid.

$\mathbf{R f}=0.21($ heptane/AcOEt: $70 / 30) .{ }^{1} \mathbf{H}$ NMR $\left(300 \mathrm{MHz}, \mathrm{CDCl}_{3}\right) \delta 7.61(\mathrm{~d}, 1 \mathrm{H}, J=7.6 \mathrm{~Hz})$, $7.20(\mathrm{~d}, 1 \mathrm{H}, J=7.6 \mathrm{~Hz}), 4.72(\mathrm{~d}, 1 \mathrm{H}, J=7.6 \mathrm{~Hz}), 4.58(\mathrm{~d}, 1 \mathrm{H}, J=7.6 \mathrm{~Hz}), 2.91(\mathrm{t}, 2 \mathrm{H}, J=$ $6.1 \mathrm{~Hz}$ ), $2.72(\mathrm{~d}, 2 \mathrm{H}, J=6.5 \mathrm{~Hz}), 2.09$ (quint, $2 \mathrm{H}, J=6.4 \mathrm{~Hz}), 0.40(\mathrm{~s}, 9 \mathrm{H}) .{ }^{13} \mathbf{C}$ NMR (50 $\left.\mathrm{MHz}, \mathrm{CDCl}_{3}\right) \delta 200.0,162.1,147.3,140.1,139.4,132.6,128.1,63.4,49.7,40.9,31.1,0.7$. IR $\left(\mathrm{CHCl}_{3}\right): v=3727,3378,2924,2853,2359,2341,2094,1670,1456,1248,1077,840,668$ $\mathrm{cm}^{-1}$. MS (apparatus 5): $\left(\mathrm{C}_{14} \mathrm{H}_{20} \mathrm{O}_{2} \mathrm{SiNa}\right)^{+}$calc. : 271.11, found 271.10.

\section{Synthesis of 54 from 51 anti}

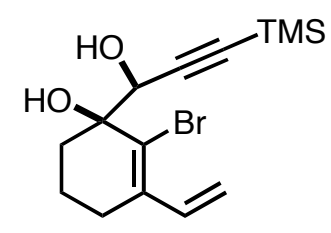

51 anti

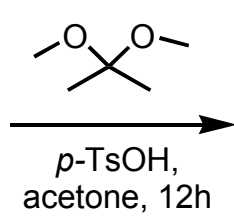

acetone, $12 \mathrm{~h}$

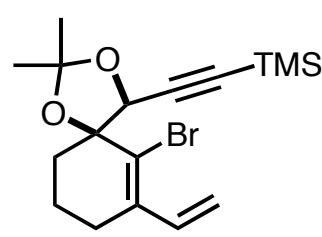

52

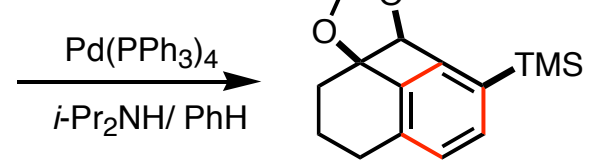

53

$\left\{\left[4 S^{*}, 5 S^{*}\right)\right.$-6-bromo-2,2-dimethyl-7-vinyl-1,3-dioxaspiro[4,5]dec-6-en4] ethynyl (trimethyl)silane (52)

Compound 52 was prepared following the same procedure than for the preparation of $\mathbf{5 8}$, starting from compound $\mathbf{5 1 \text { anti }}(50 \mathrm{mg}, 1.51 \mathrm{mmol}, 1$ eq.), 2,2-dimethoxypropane ( $2 \mathrm{~mL}$, large exces), and $p$-TsOH (0.1 eq.) in acetone $(5 \mathrm{~mL})$ during $1 \mathrm{~h}$. The same aquaous treatment were realized and the crude product was purified by chromatography on silica gel (elution with heptane/AcOEt: 98/2) to give $55 \mathrm{mg}(98 \%$ ) of the product $\mathbf{5 2}$ as a white solid. 
Rf $=0.70$ (heptane/AcOEt: 80/20). ${ }^{1} \mathbf{H}$ NMR $\left(300 \mathrm{MHz}, \mathrm{CDCl}_{3}\right) \delta 6.93(\mathrm{dd}, 1 \mathrm{H}, J=11.1 \mathrm{~Hz}$, $J=17.1 \mathrm{~Hz}), 5.33(\mathrm{~d}, 1 \mathrm{H}, J=17.1 \mathrm{~Hz}), 5.23(\mathrm{~d}, 1 \mathrm{H}, J=11.2 \mathrm{~Hz}, 4.70(\mathrm{~d}, 1 \mathrm{H}), 2.26(\mathrm{t}, 2 \mathrm{H}, J=$ $7.0 \mathrm{~Hz}), 2.15-1.96(\mathrm{~m}, 4 \mathrm{H}), 1.69(\mathrm{~s}, 3 \mathrm{H}), 1.38(\mathrm{~s}, 3 \mathrm{H}), 0.09(\mathrm{~s}, 9 \mathrm{H}) .{ }^{13} \mathbf{C}$ NMR $(75 \mathrm{MHz}$, $\left.\mathrm{CDCl}_{3}\right) \delta 137.8,136.6,127.3,116.8,110.6,99.8,94.6,85.2,72.3,38.8,26.9,26.2,26.0,19.3$, -0.5. MS (apparatus 5) : $\left(\mathrm{C}_{17} \mathrm{H}_{25} \mathrm{BrO}_{2} \mathrm{SiNa}\right)^{+}$calc.: 391.0705, found 391.0703.

[(6b $\left.S^{*}, 9 \mathrm{a} R^{*}\right)-8,8-d i m e t h y l-2,3-d i h y d r o-1 H, 6 \mathrm{~b} H$-naphto[1',8' $\left.: 2,3,4\right]$ cyclobutan[1,2d] $[1,3]$ dioxol-6-yl](trimethyl)silane (53)

Compound $\mathbf{5 3}$ was prepared following the same procedure than for the preparation of $\mathbf{1 0}$, starting from compound $\mathbf{5 2}$ (26 mg, $0.07 \mathrm{mmol}$, 1eq.), $\mathrm{Pd}\left(\mathrm{PPh}_{3}\right)_{4}(8 \mathrm{mg}, 0.007 \mathrm{mmol}, 0.1$ eq.) and diisopropyl amine $(0.2 \mathrm{~mL})$ in benzene $(0.6 \mathrm{~mL})$. After chromatography on silica gel (elution with heptane/AcOEt: 95/5), $13 \mathrm{mg}$ (66\%) of product 53 was obtained as a white solid.

$\mathbf{R f}=0.80\left(\right.$ heptane/AcOEt: 70/30). ${ }^{1} \mathbf{H}$ NMR $\left(300 \mathrm{MHz}, \mathrm{CDCl}_{3}\right) \delta 7.31(\mathrm{~d}, 1 \mathrm{H}, J=7.5 \mathrm{~Hz})$, $7.03(\mathrm{~d}, 1 \mathrm{H}, J=7.5 \mathrm{~Hz}), 5.25(\mathrm{~s}, 1 \mathrm{H}), 2.95(\mathrm{dd}, 1 \mathrm{H}, J=6.4 \mathrm{~Hz}, J=17.3 \mathrm{~Hz}), 2.49-1.95(\mathrm{~m}$, $5 \mathrm{H}), 1.53(\mathrm{~s}, 3 \mathrm{H}), 1.38(\mathrm{~s}, 3 \mathrm{H}), 0.30(\mathrm{~s}, 9 \mathrm{H}) .{ }^{13} \mathbf{C ~ N M R}\left(50 \mathrm{MHz}, \mathrm{CDCl}_{3}\right) \delta 153.8,147.5$, 136.5, 135.7, 134.4, 127.1, 114.2, 87.8, 85.9, 29.4, 29.1, 28.3, 25.5, 22.1, -0.9. IR (FTIR, film) : $v=3427,2954,2866,1731,1593,1456,1377,1252,1210,1154,1081,908,843,756$, $734,692 \mathrm{~cm}^{-1}$. 


\section{Spectra}
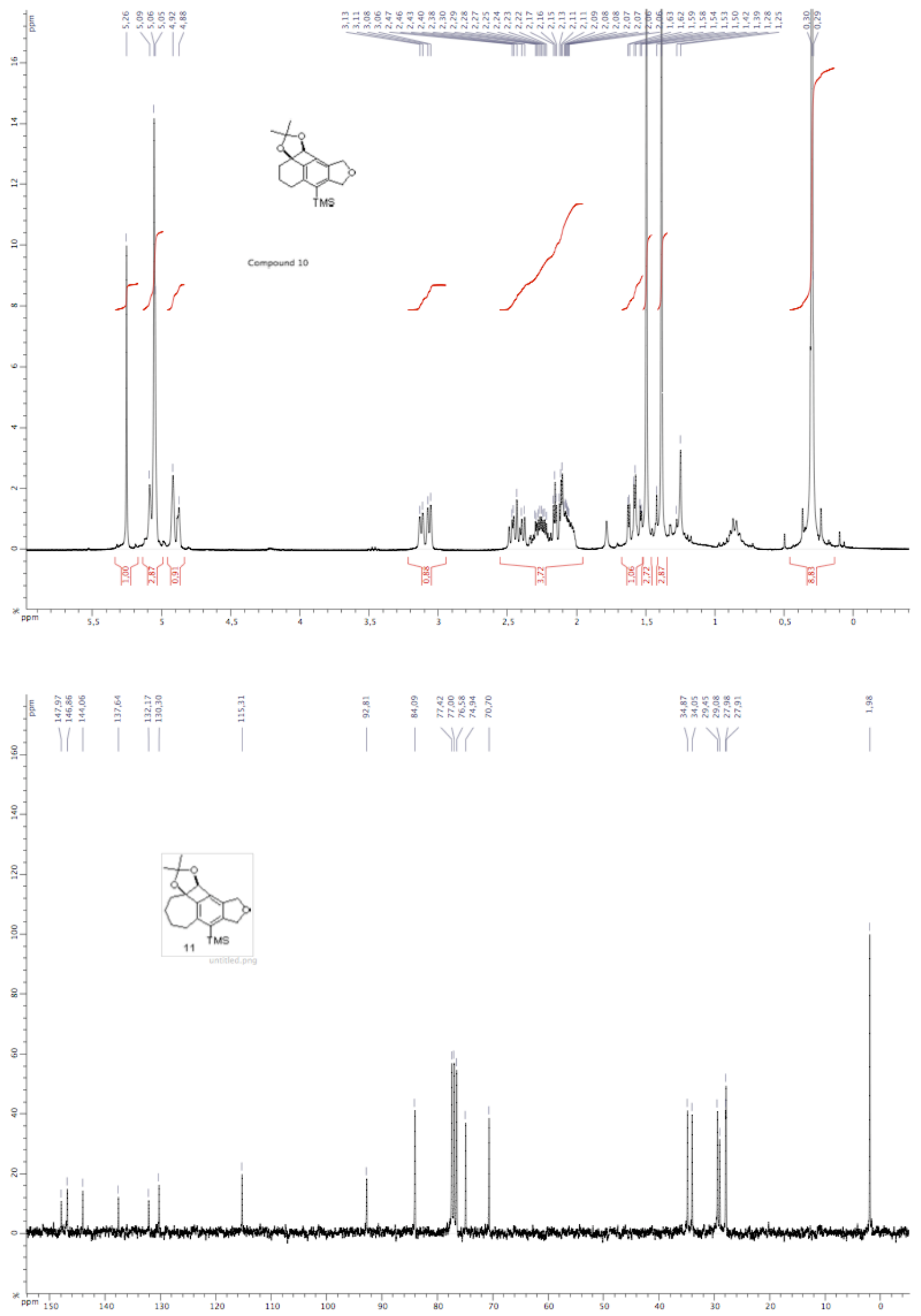

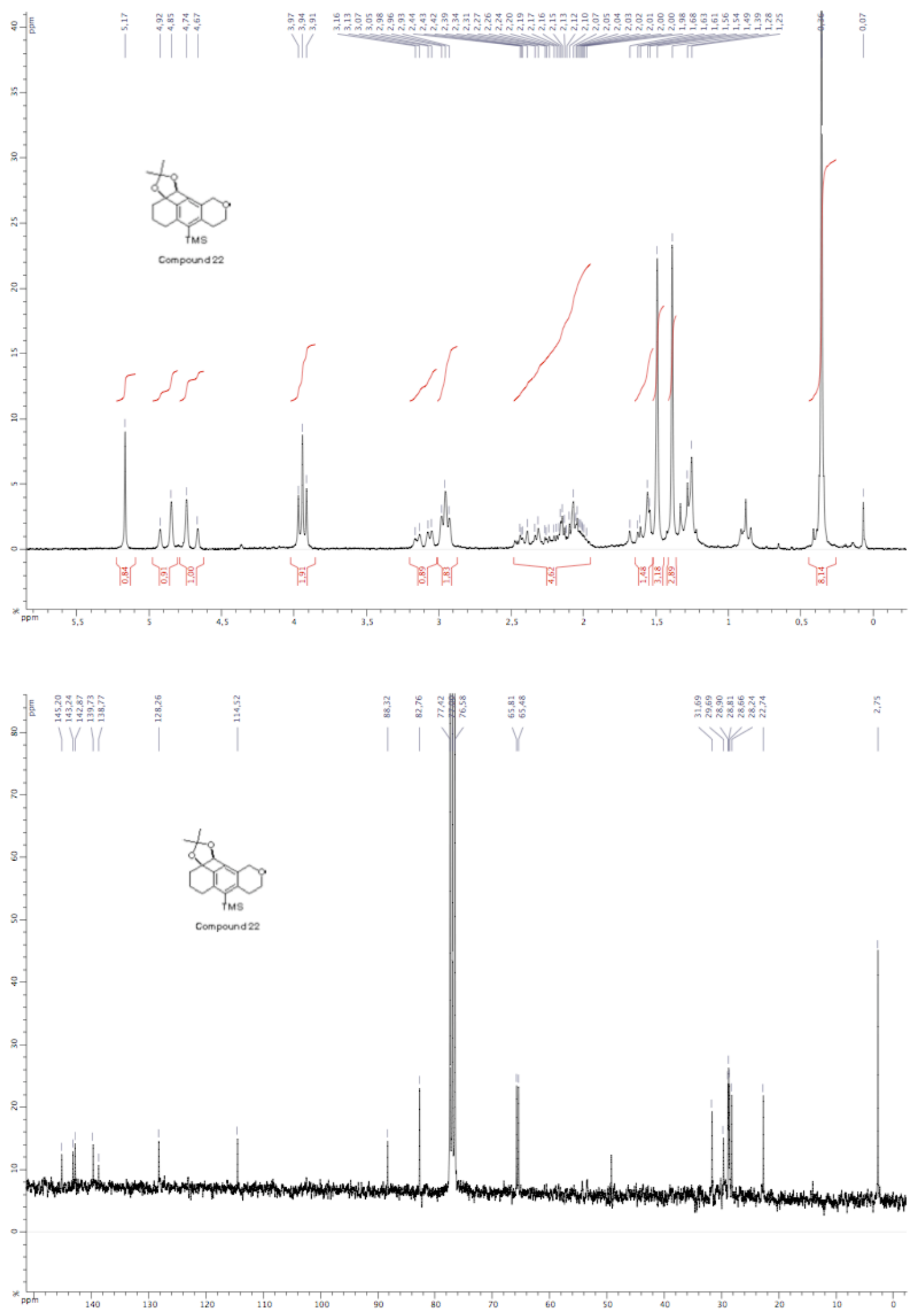

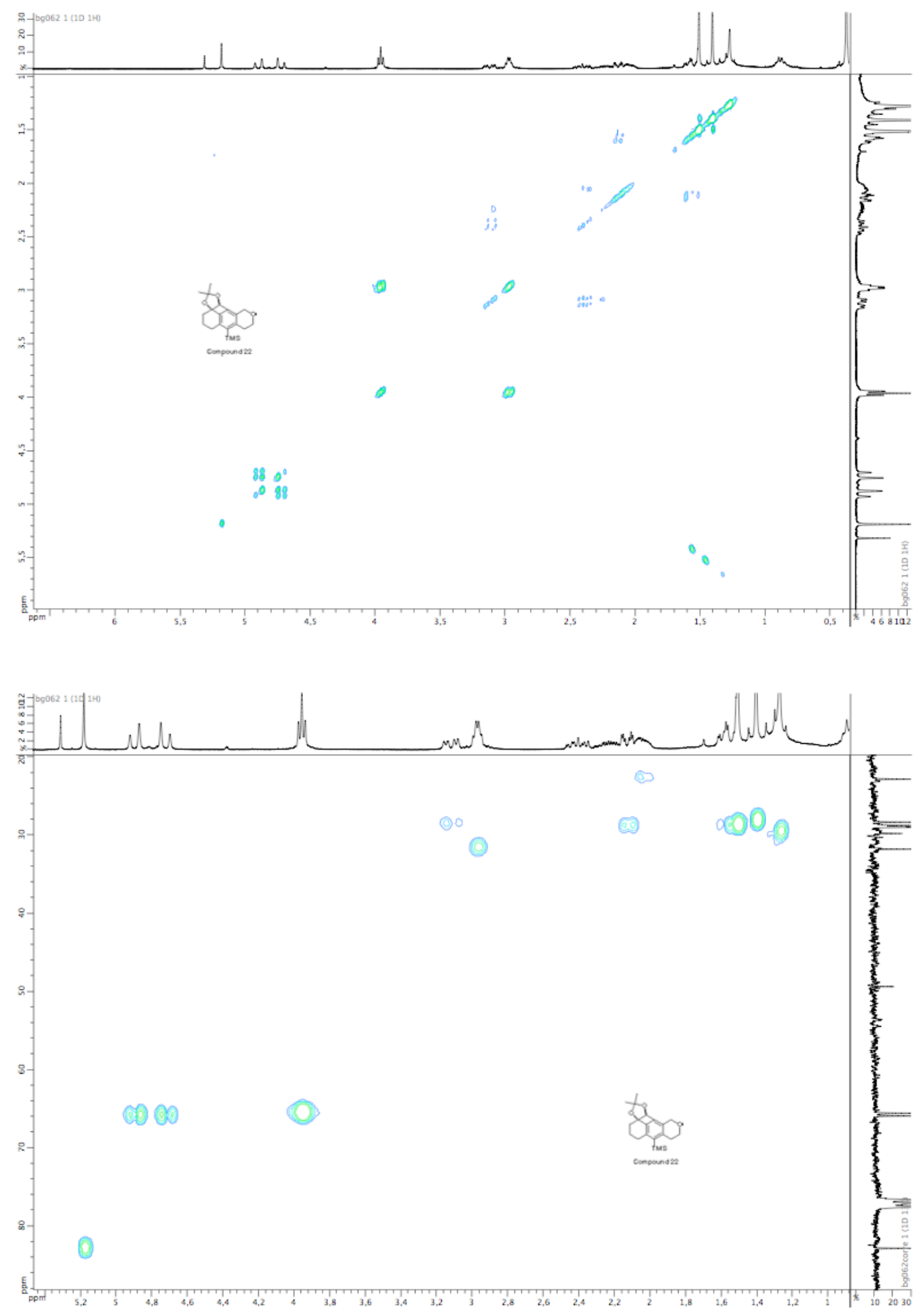

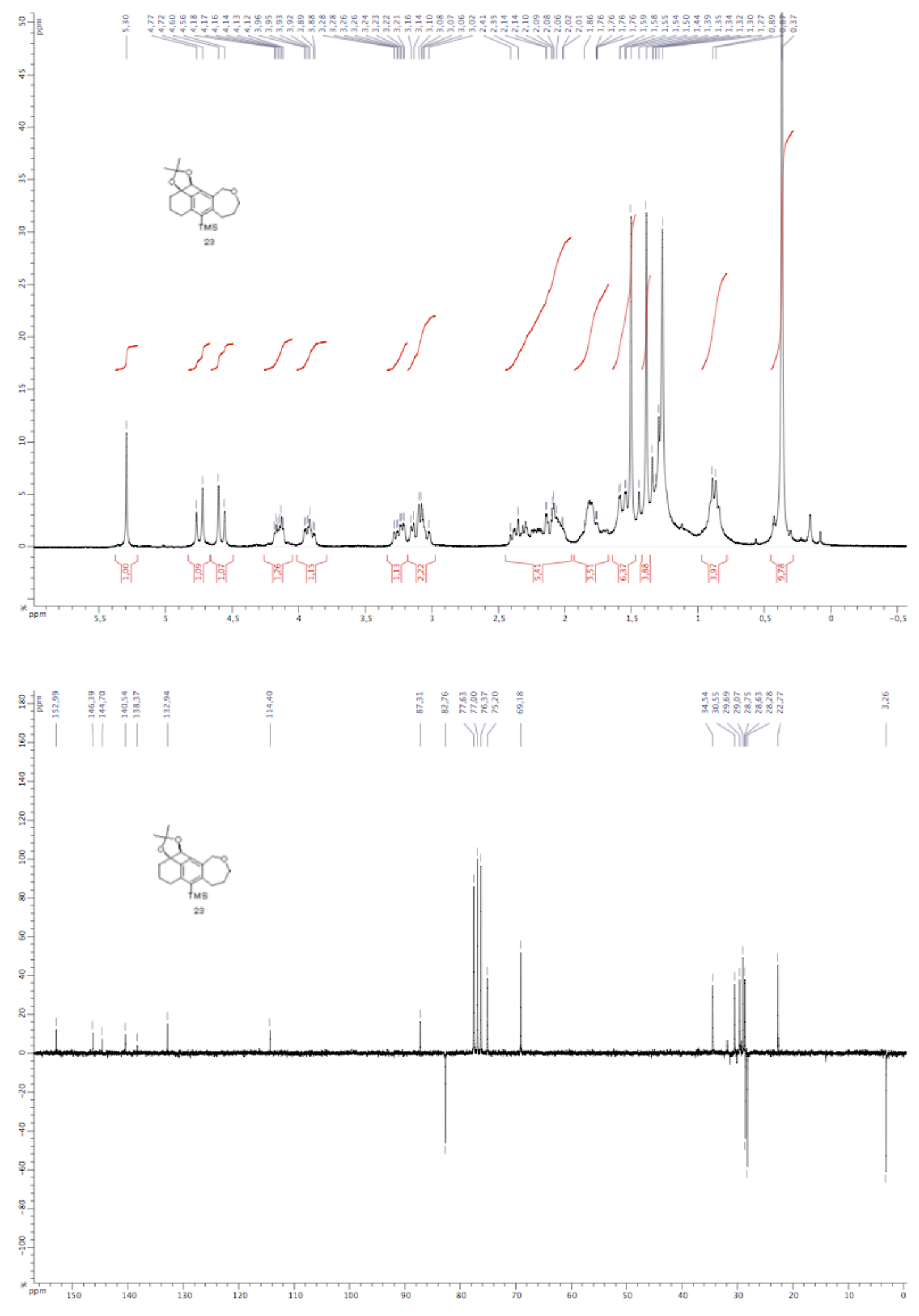

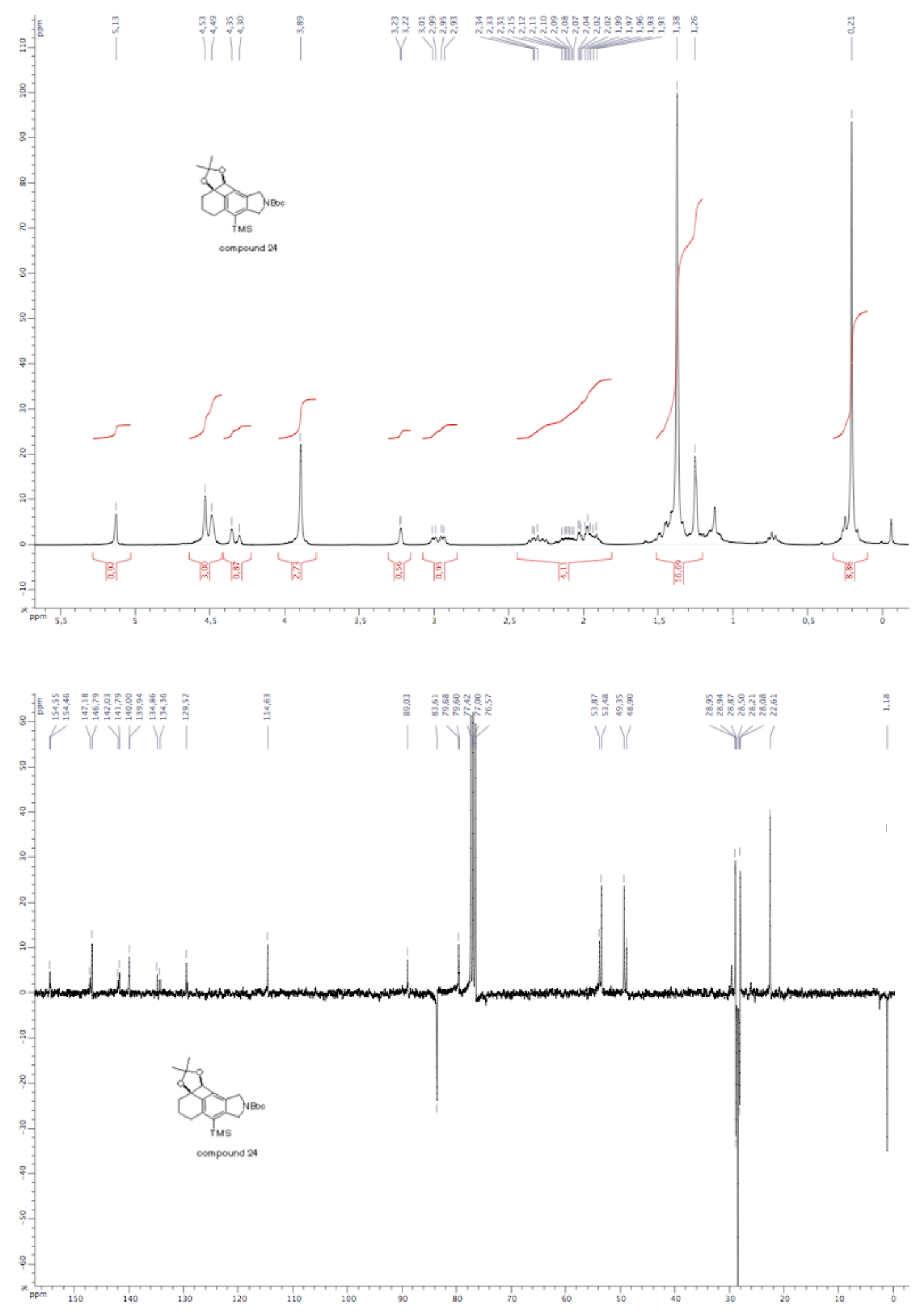

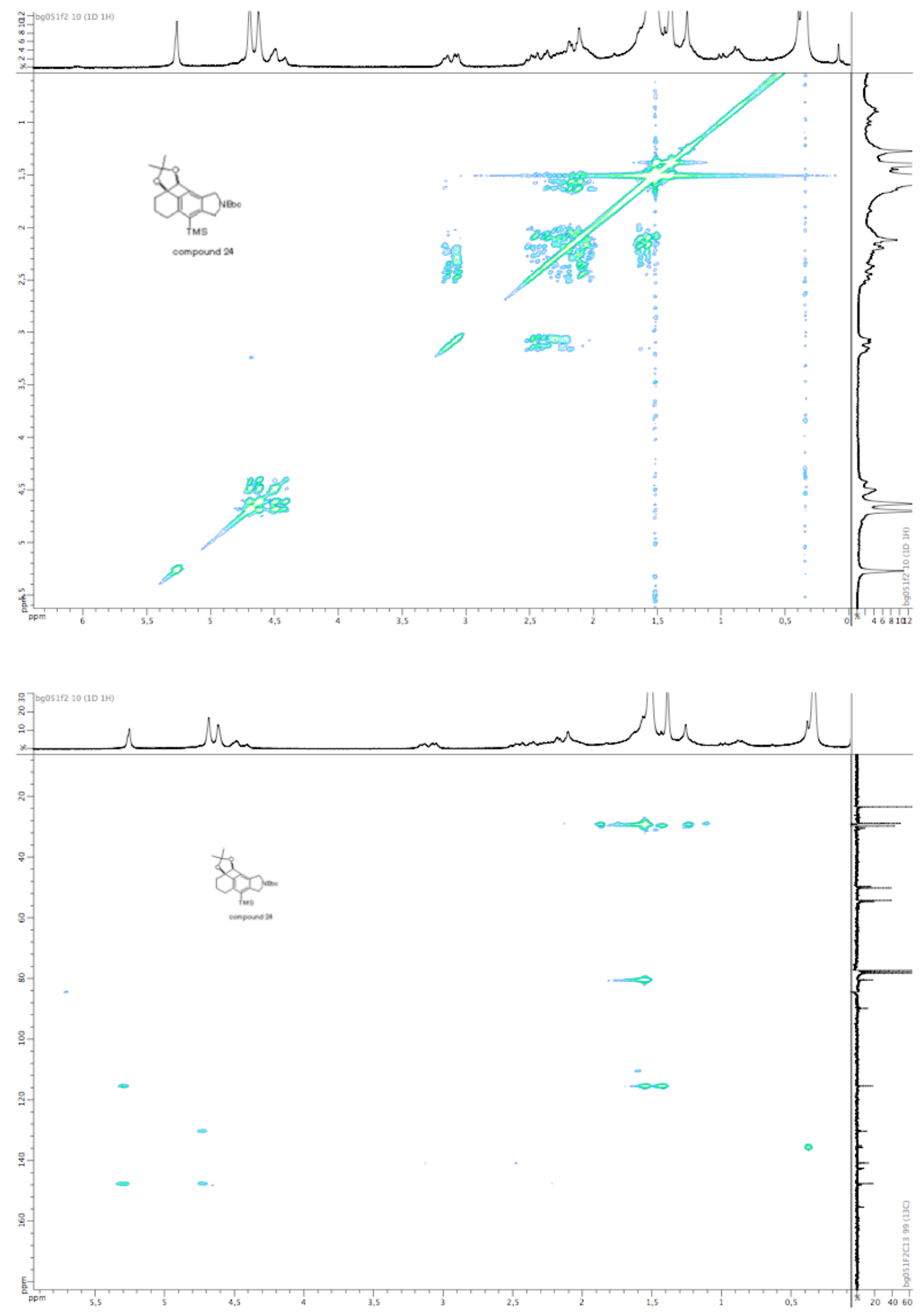

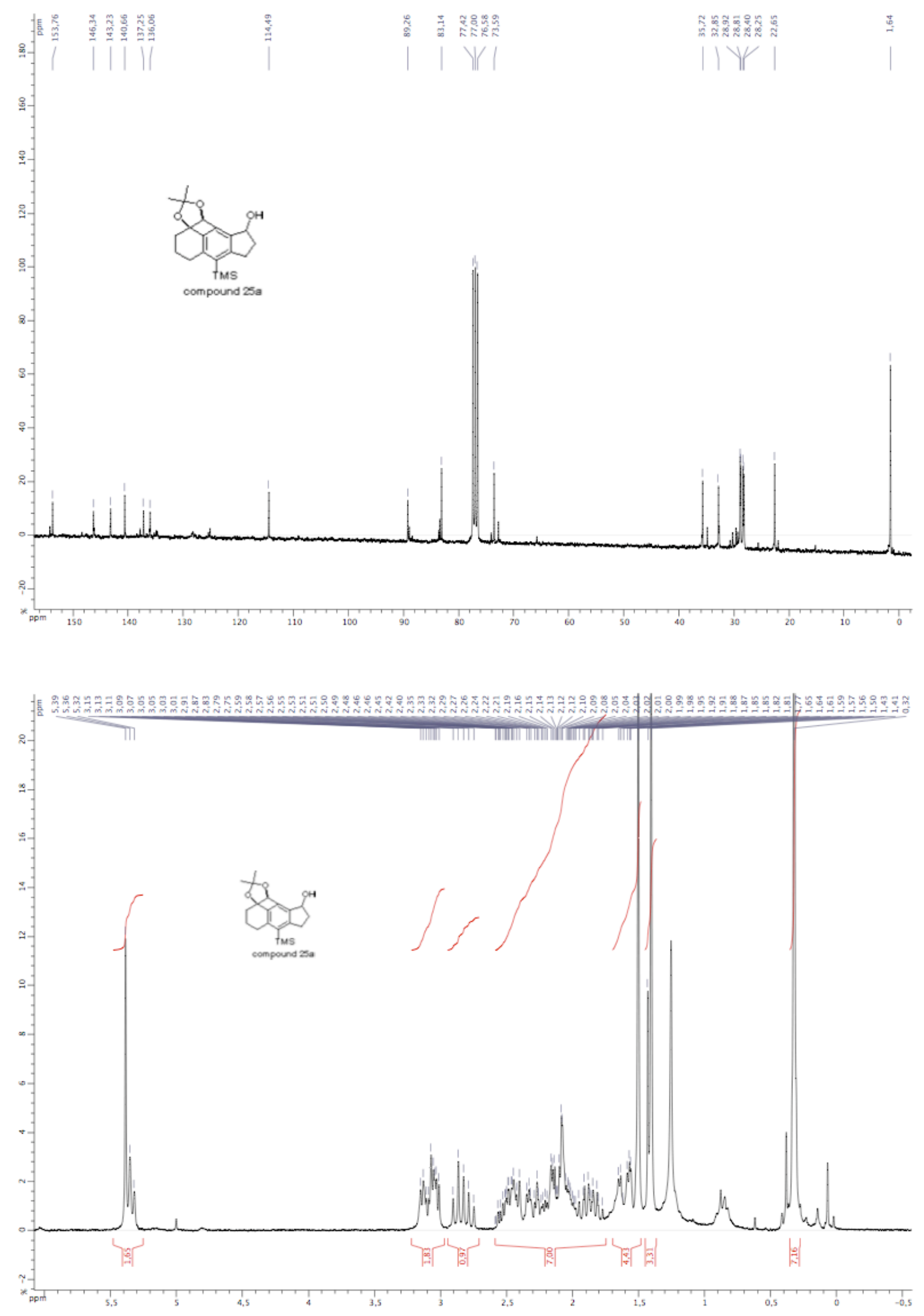

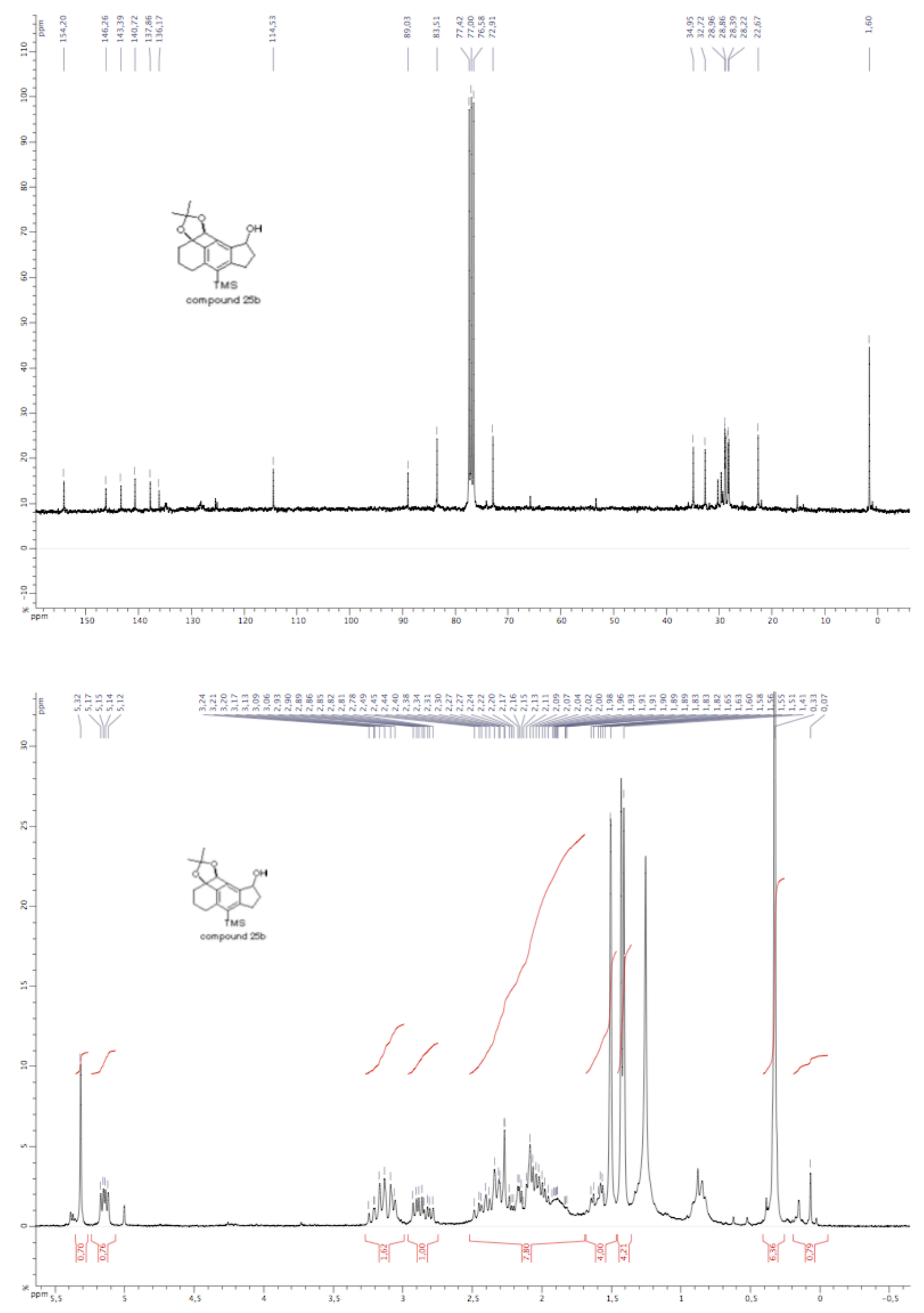

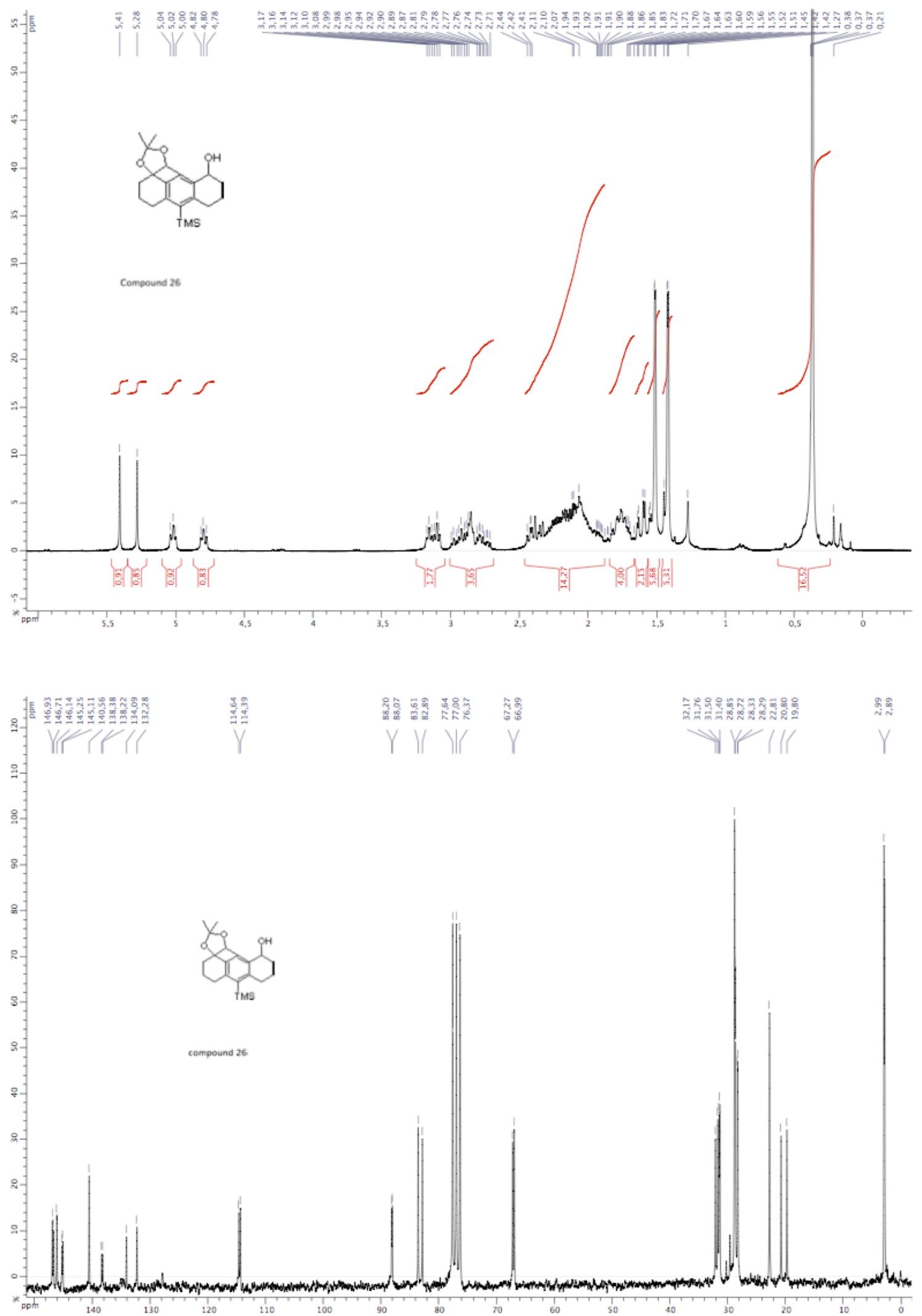

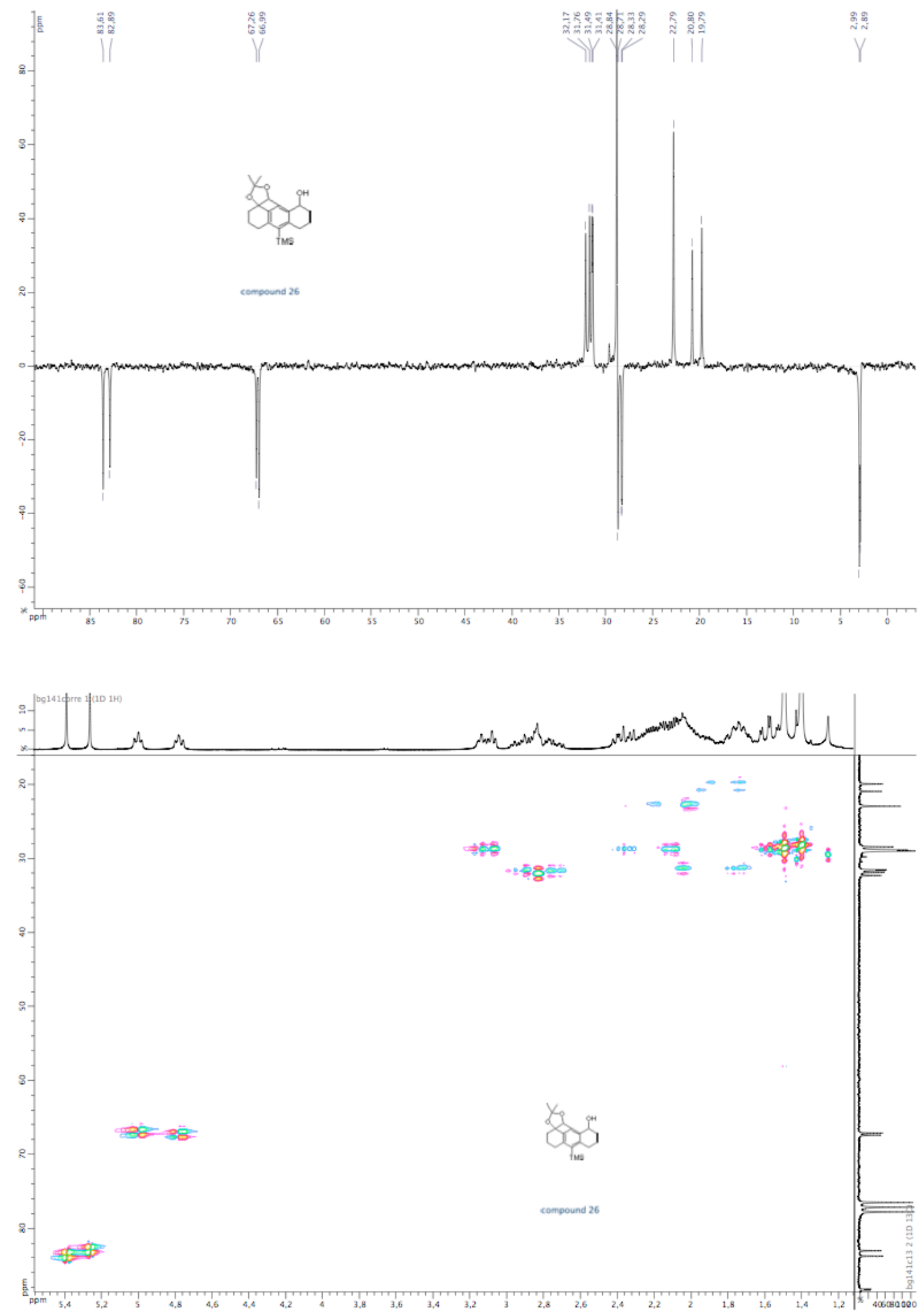

S43 


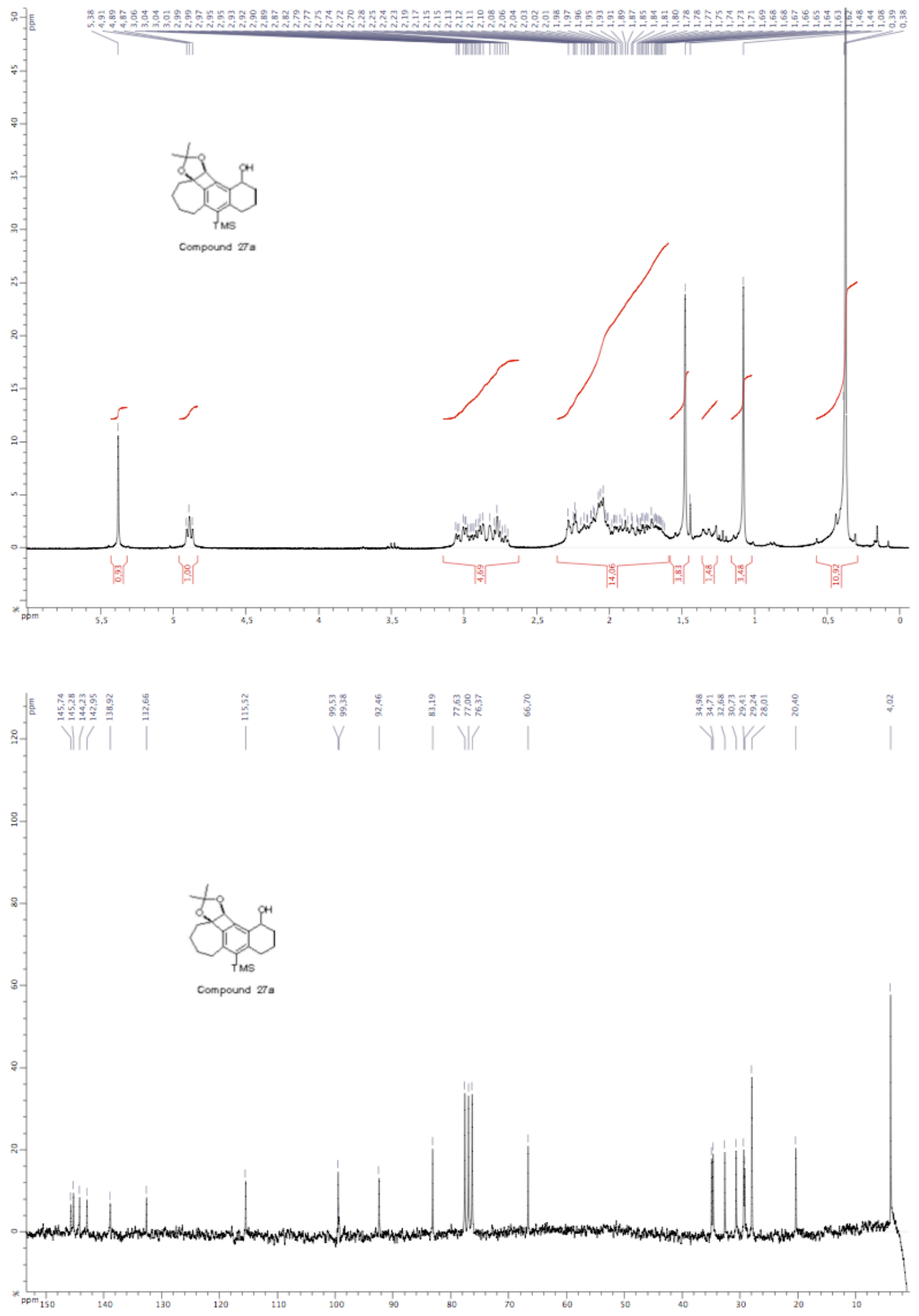



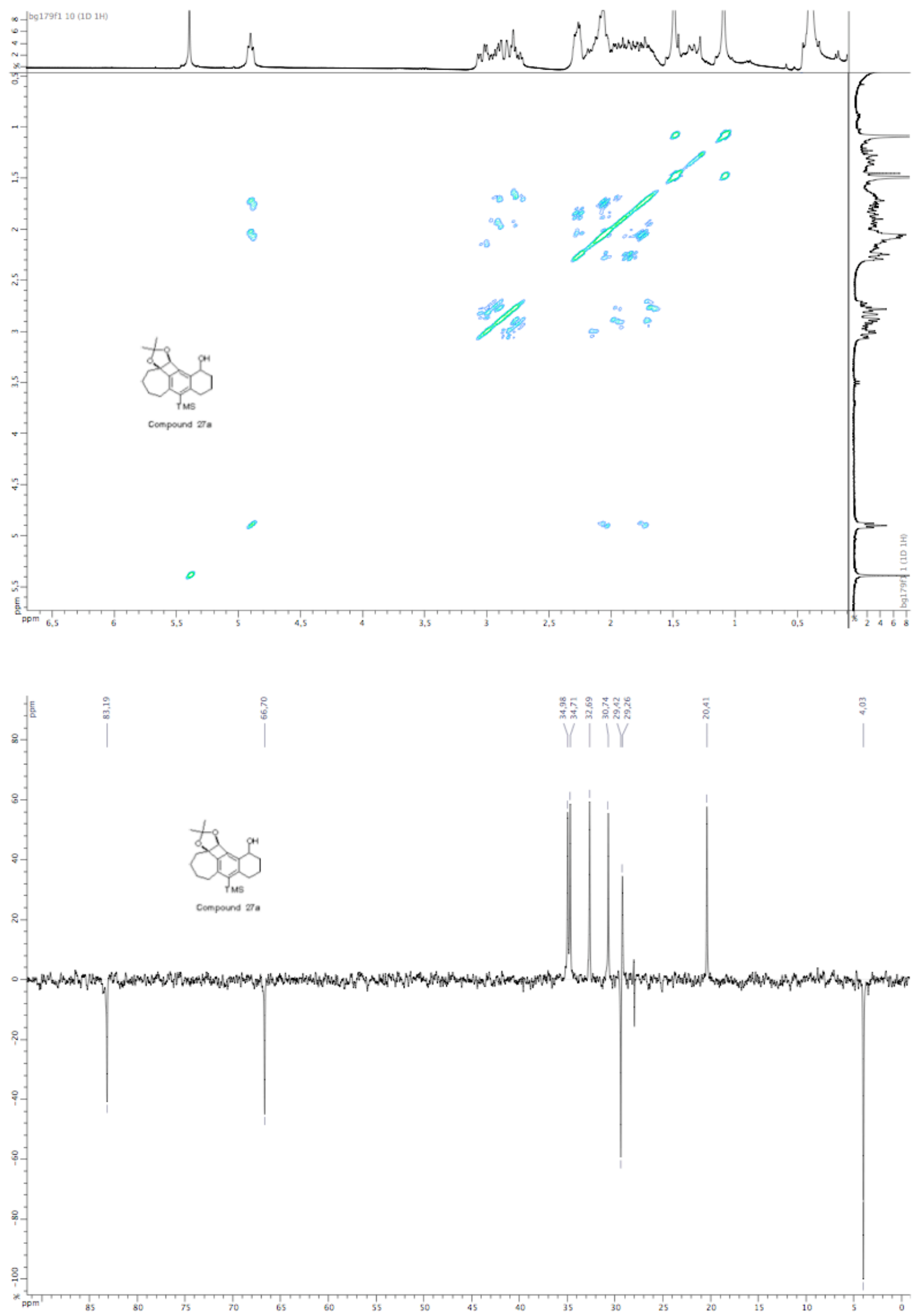

S45 

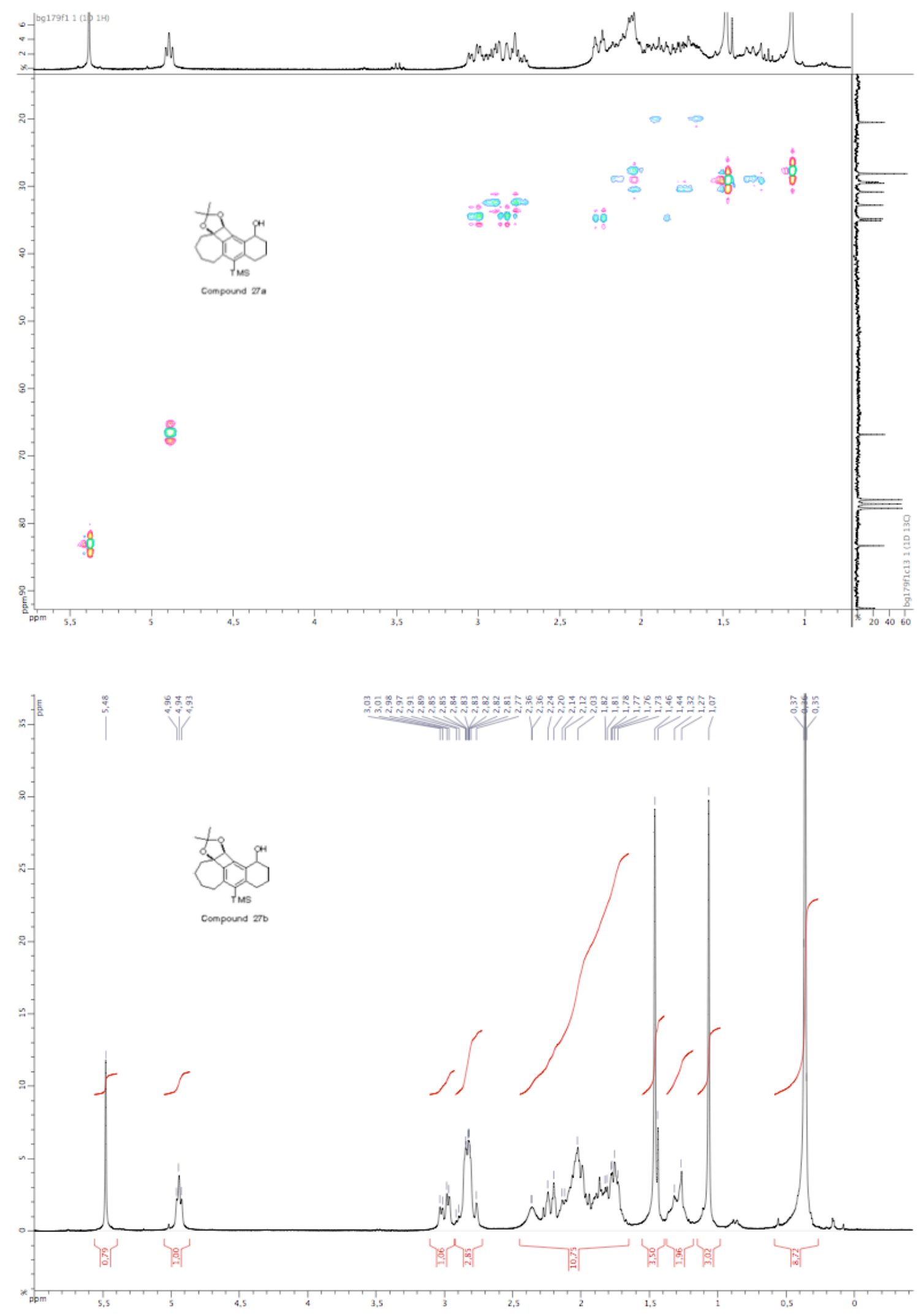

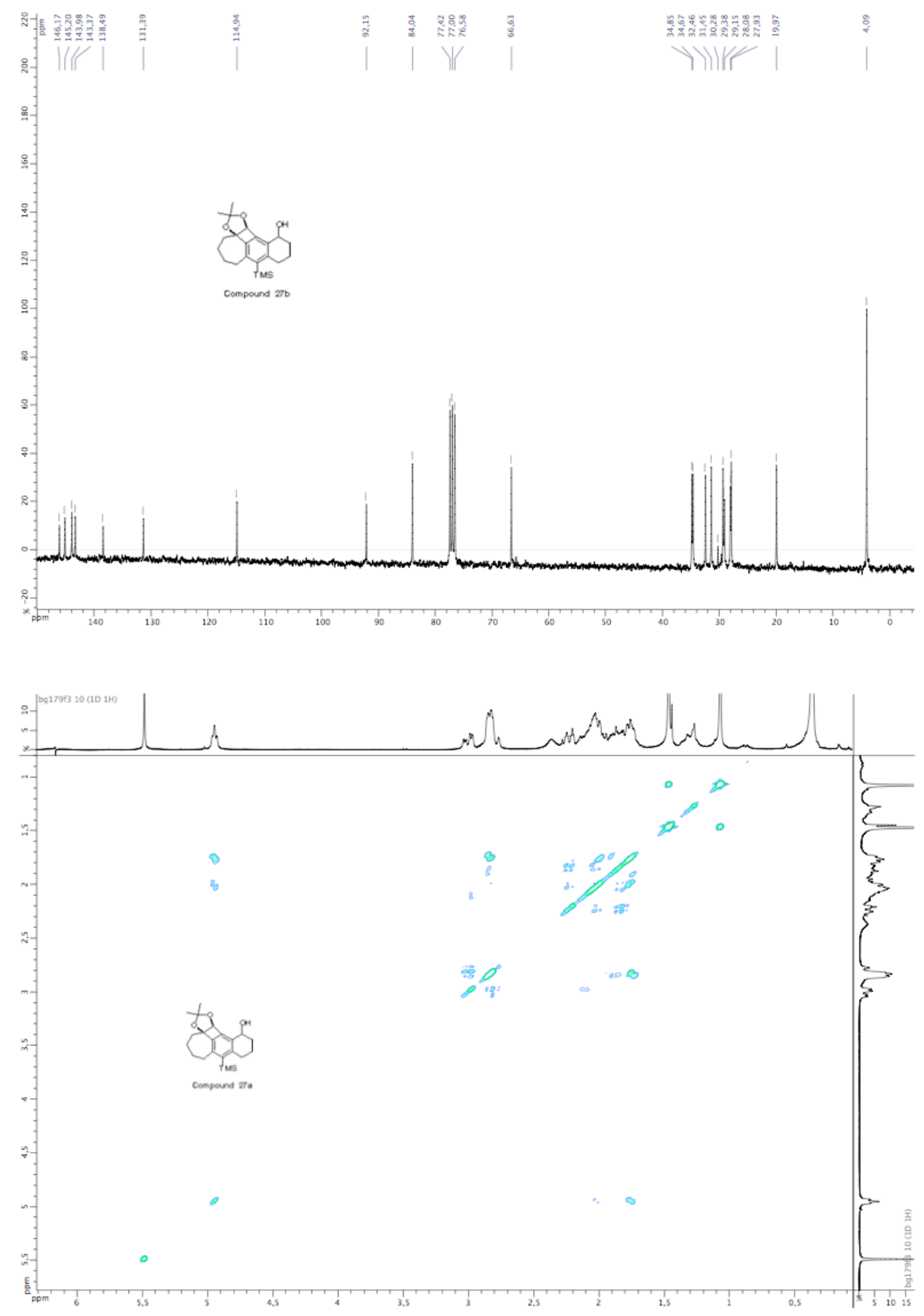

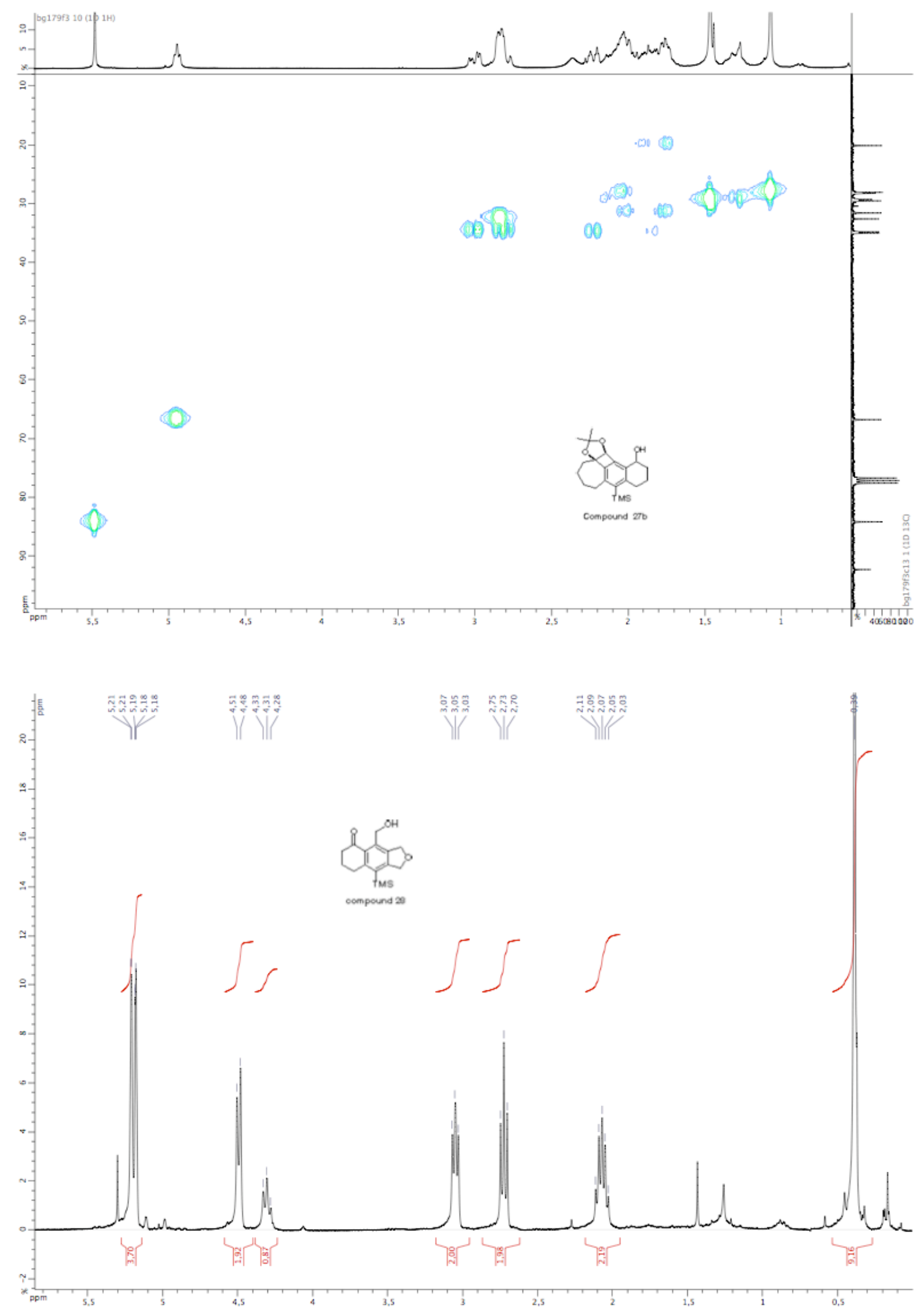

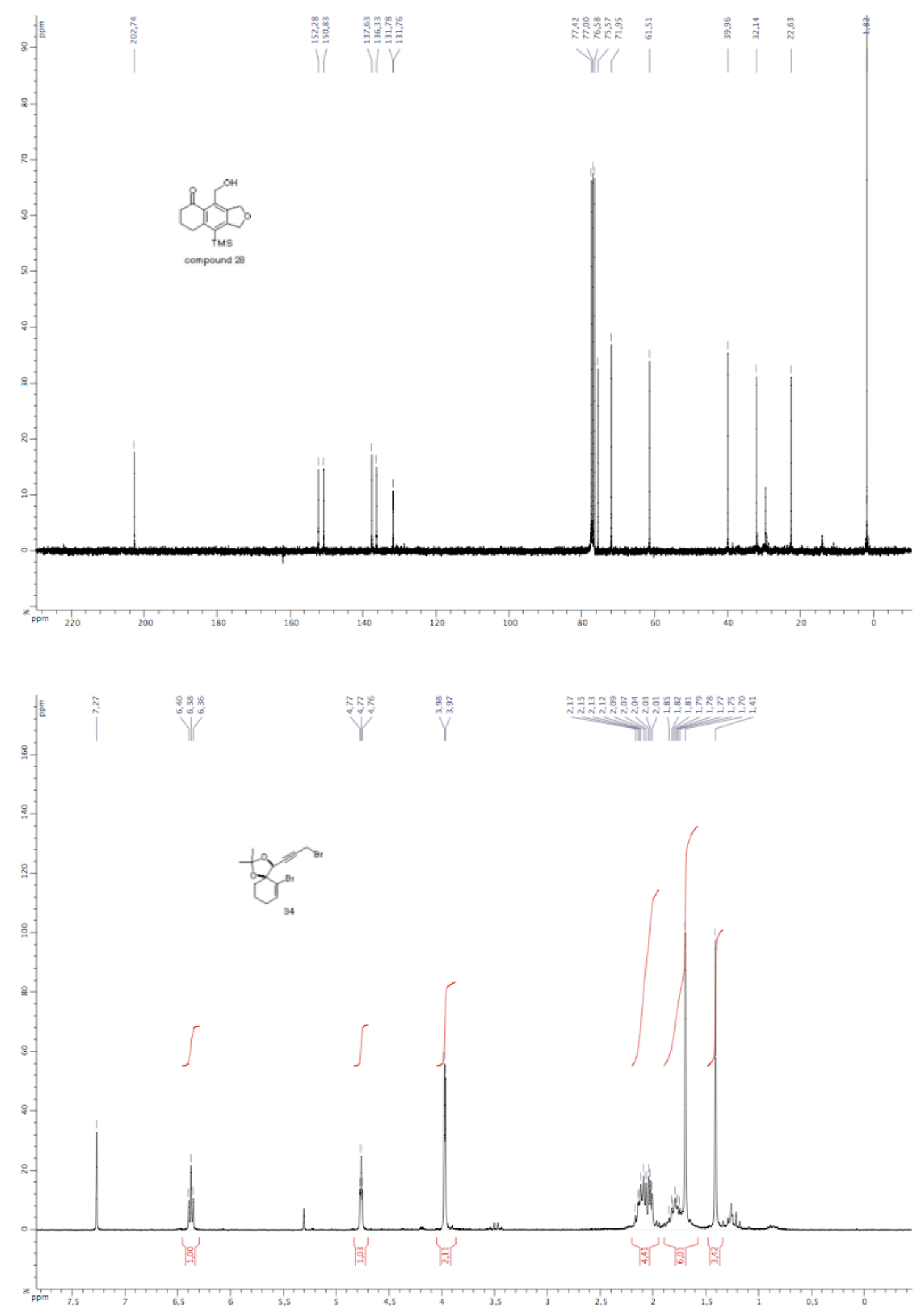

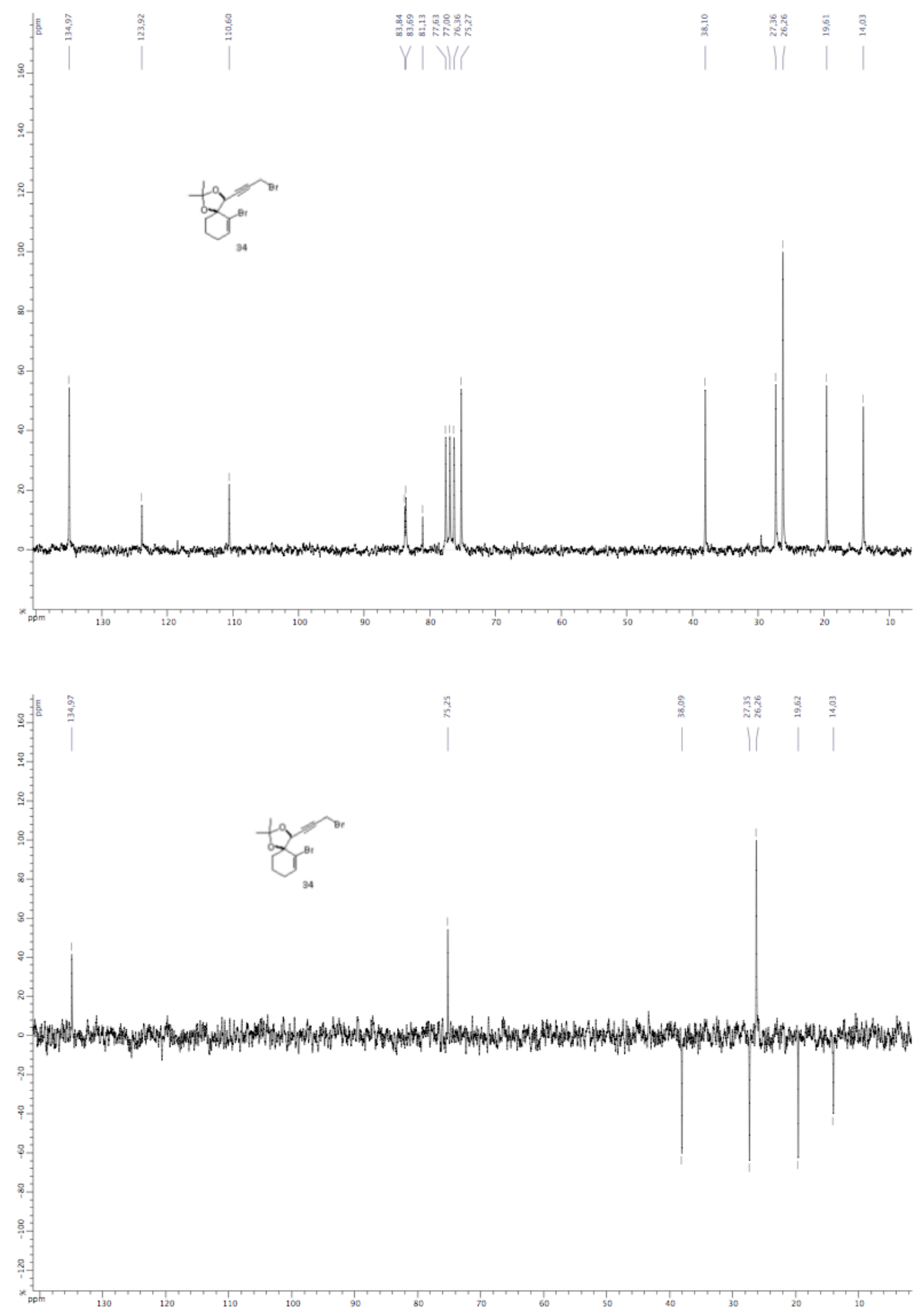

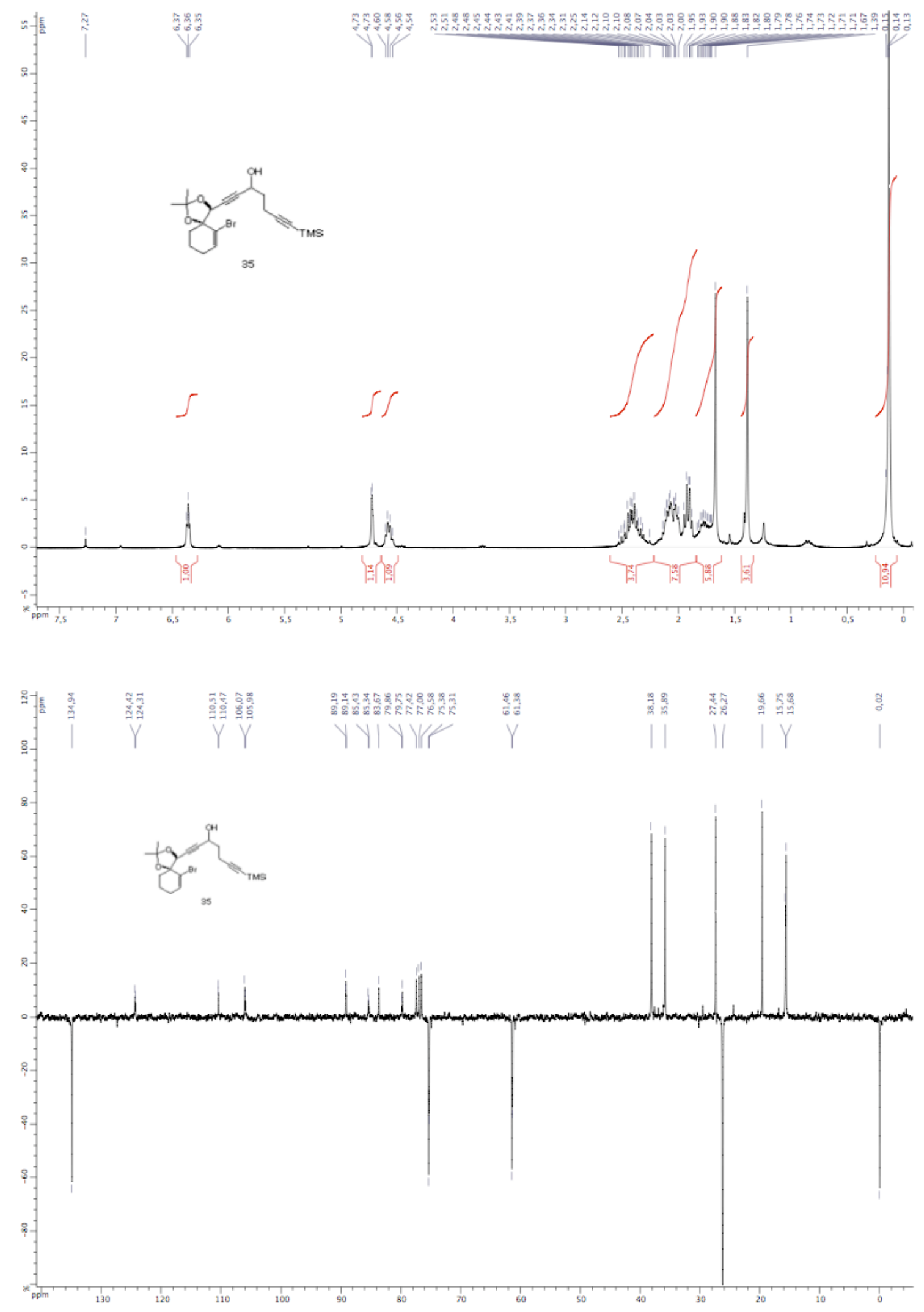

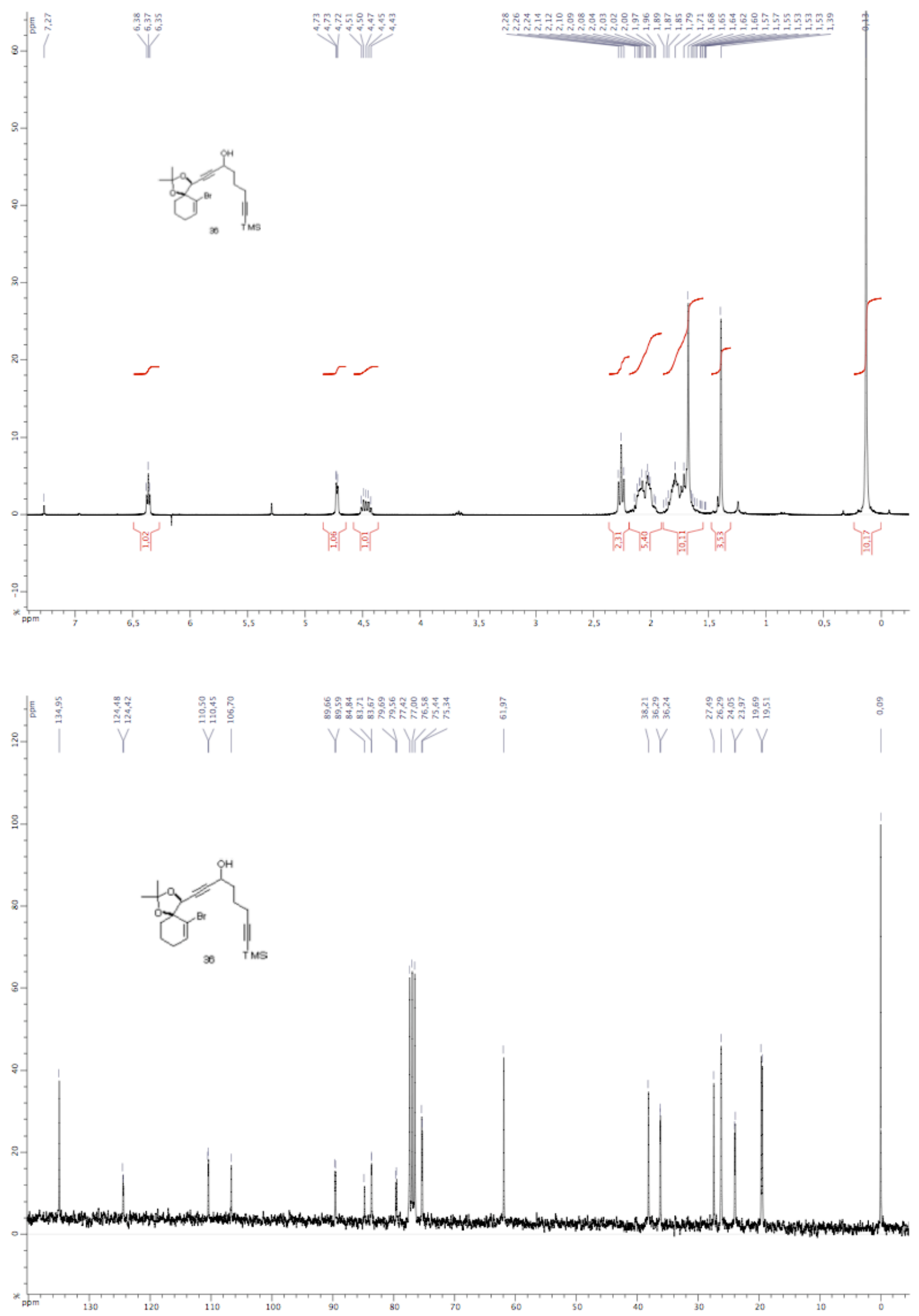

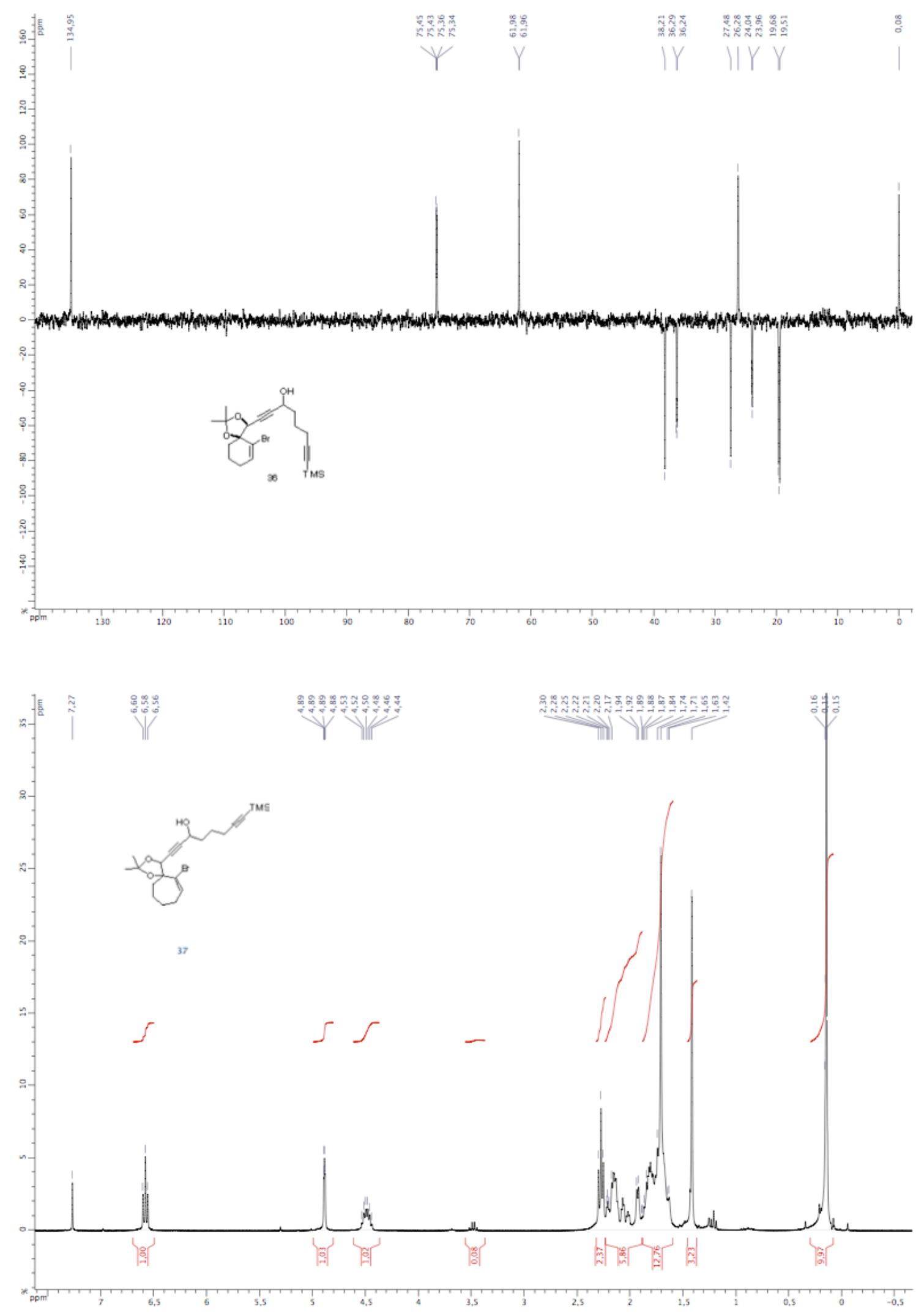

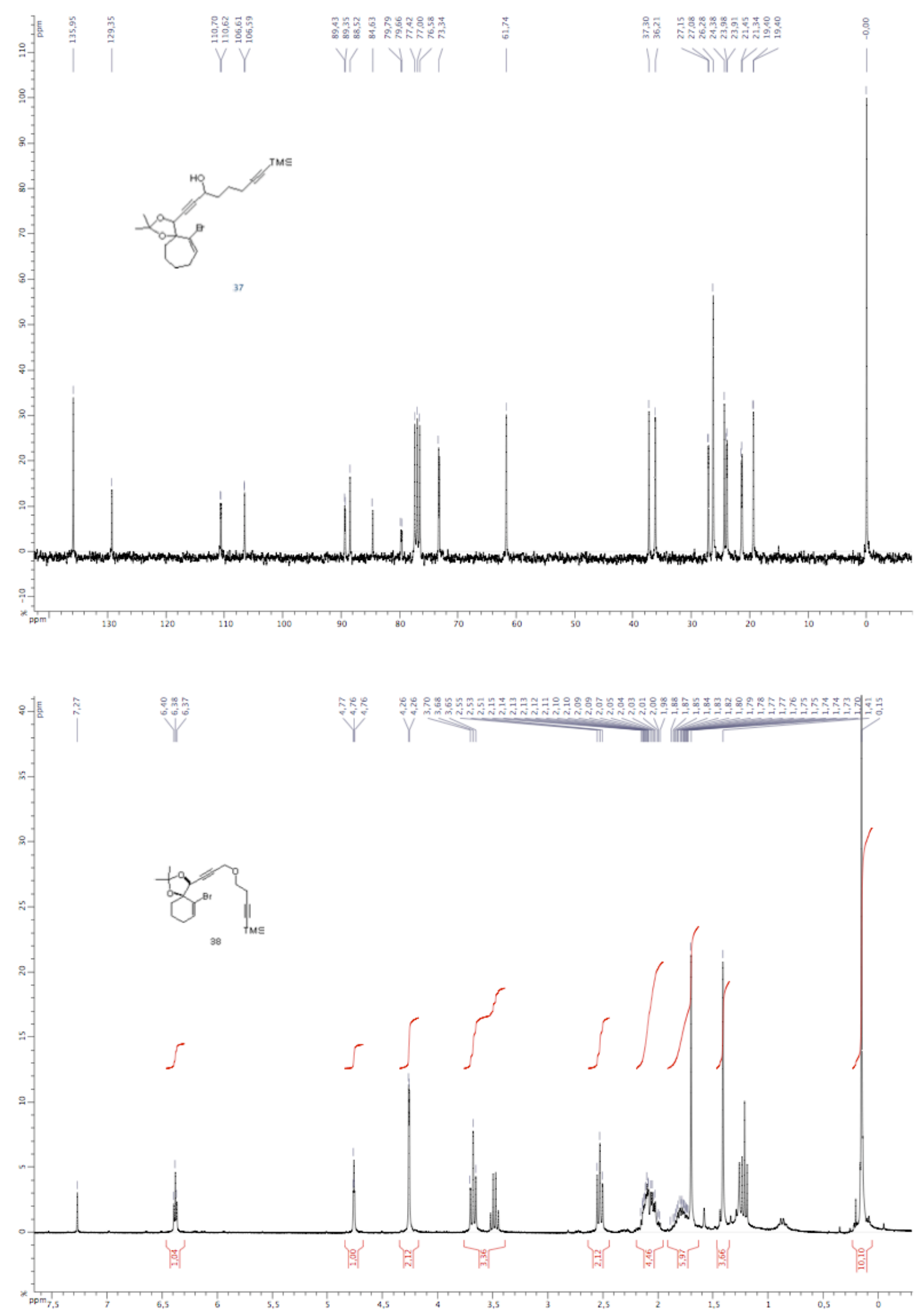

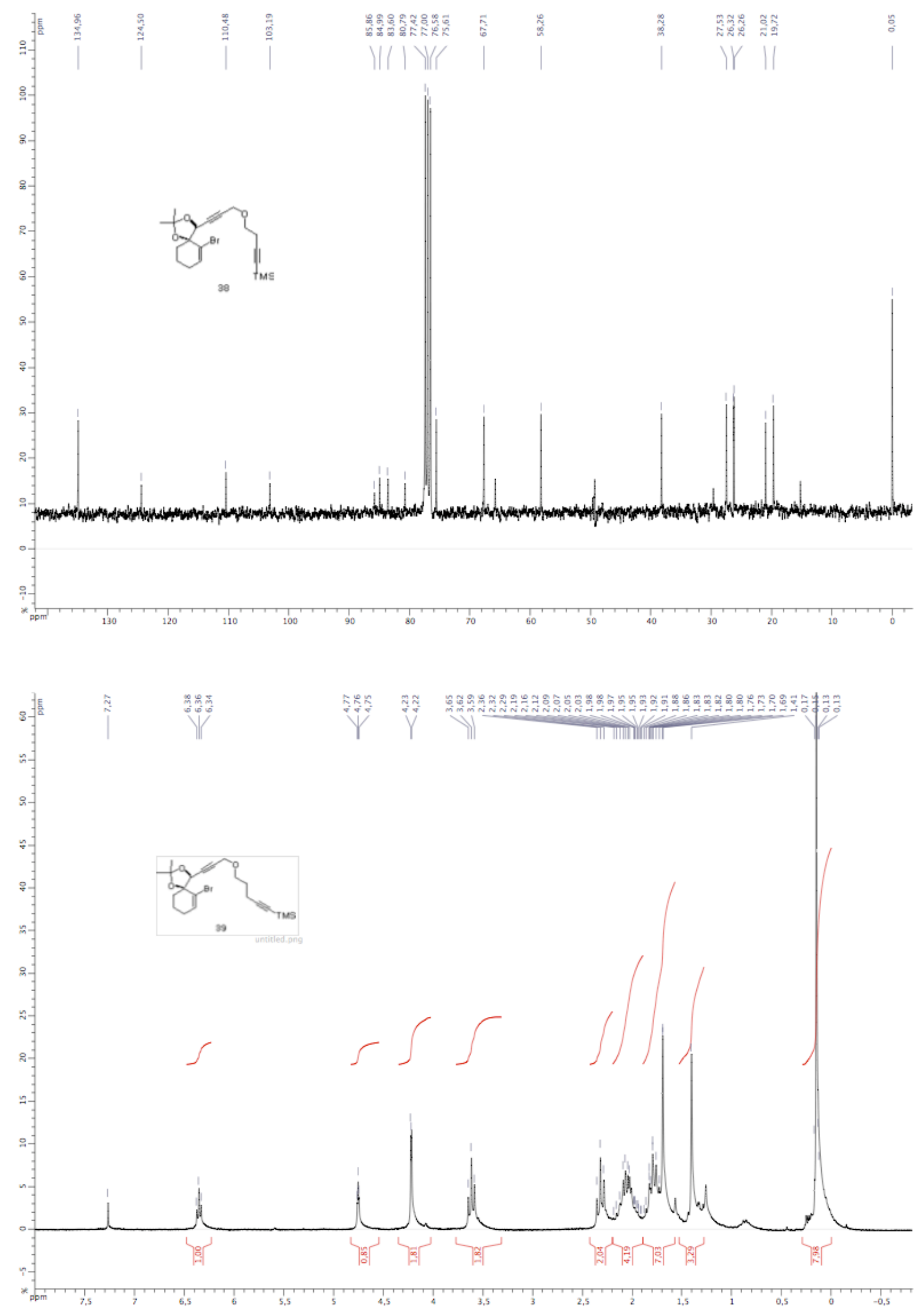

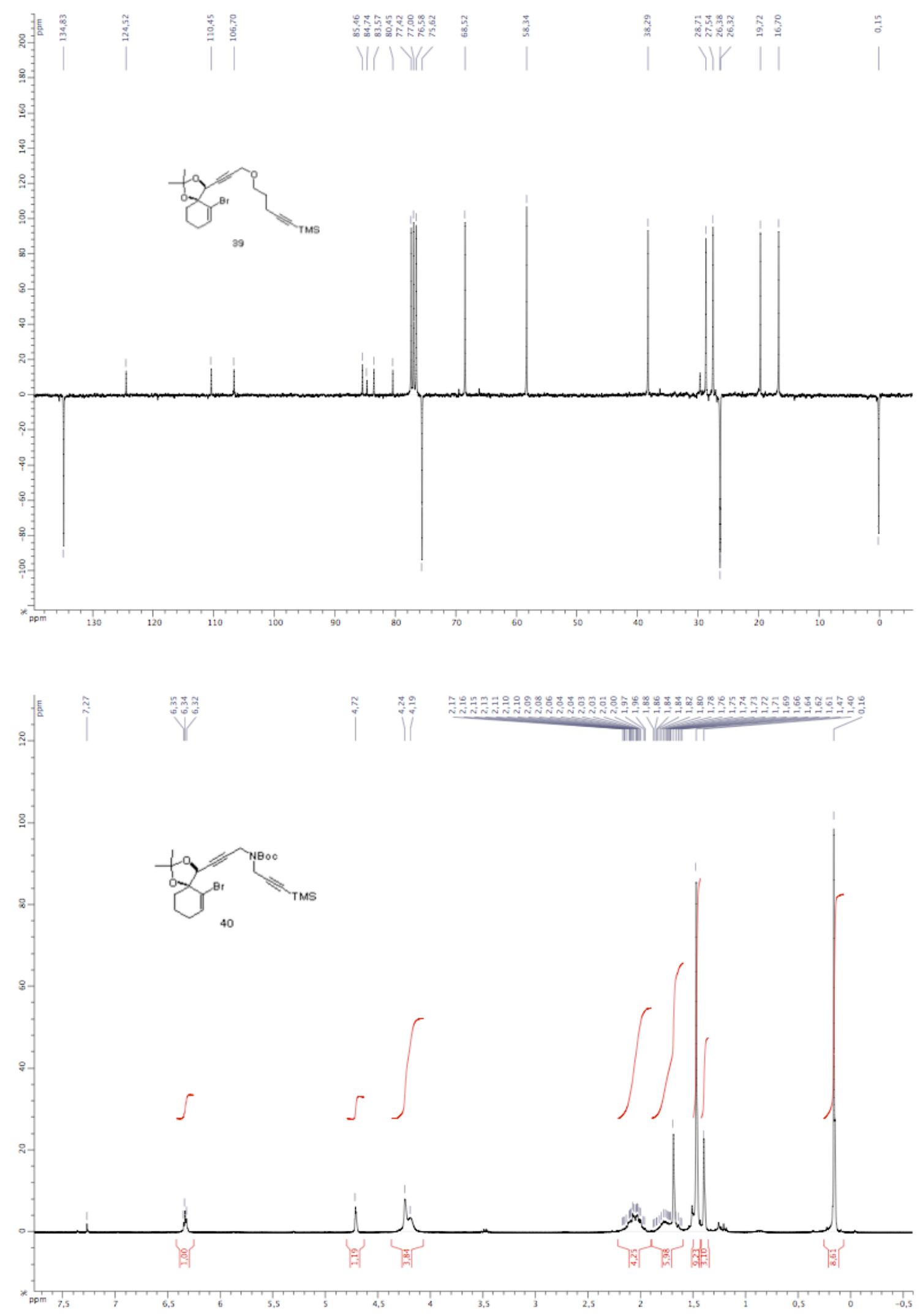

S56 

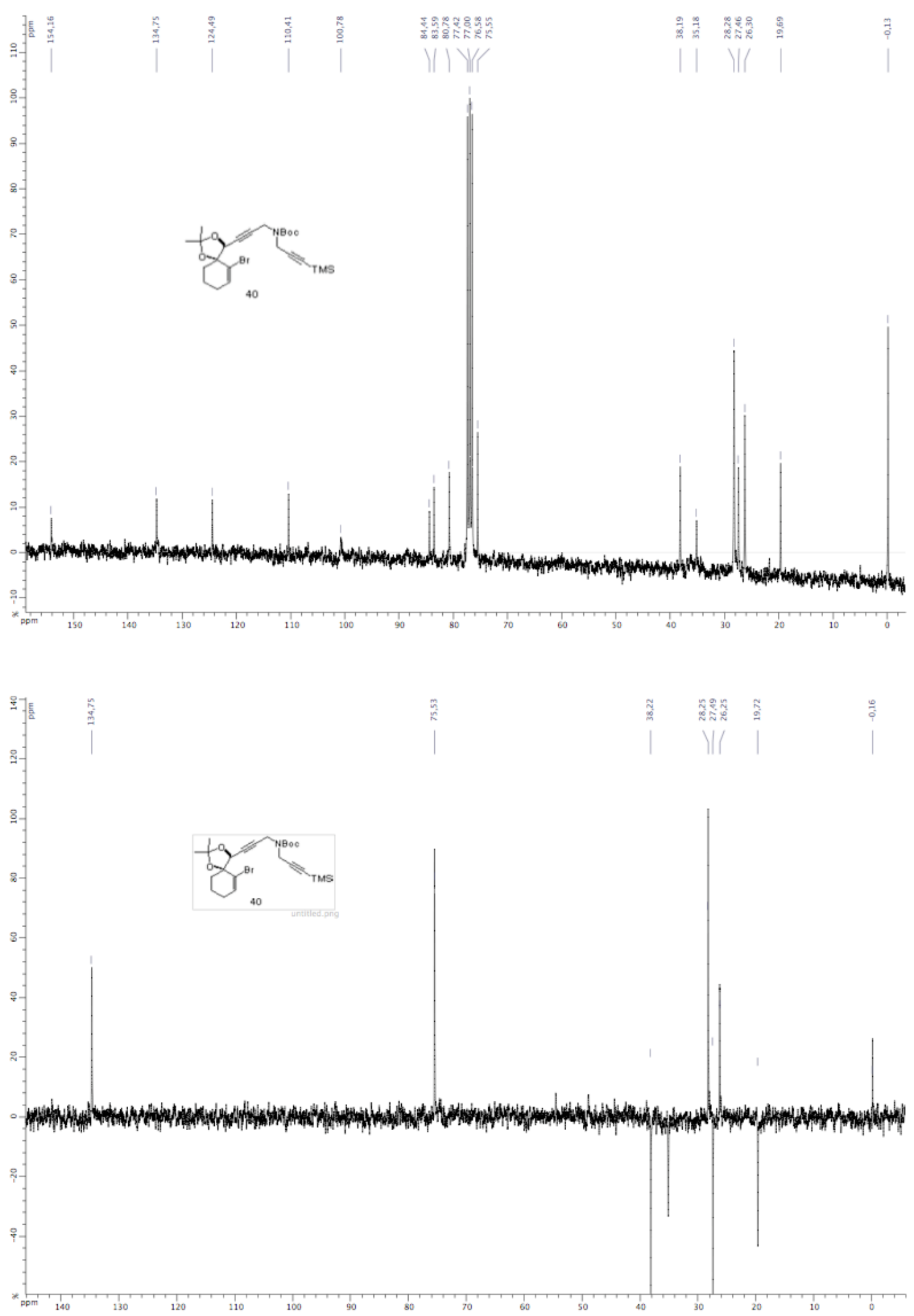

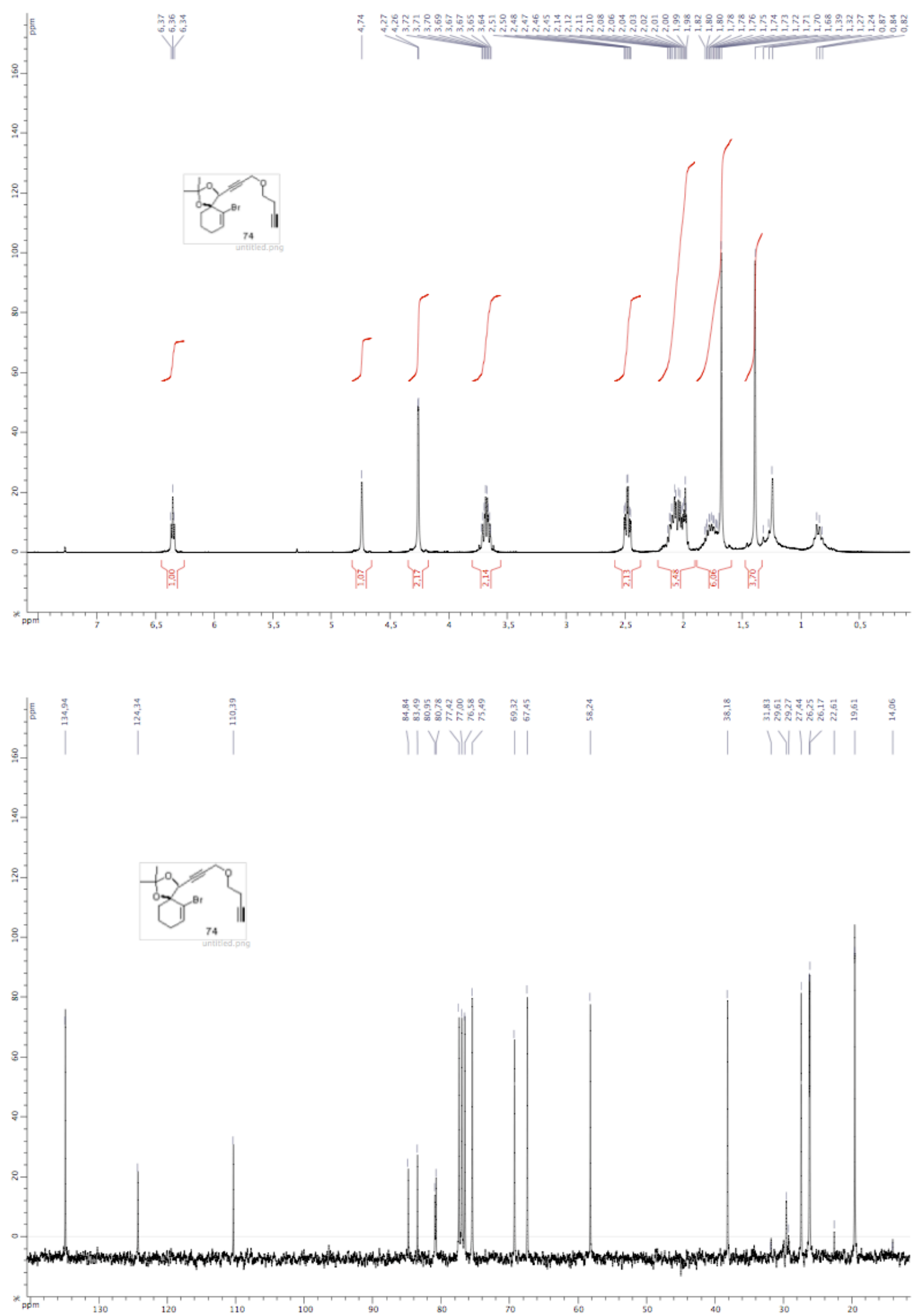

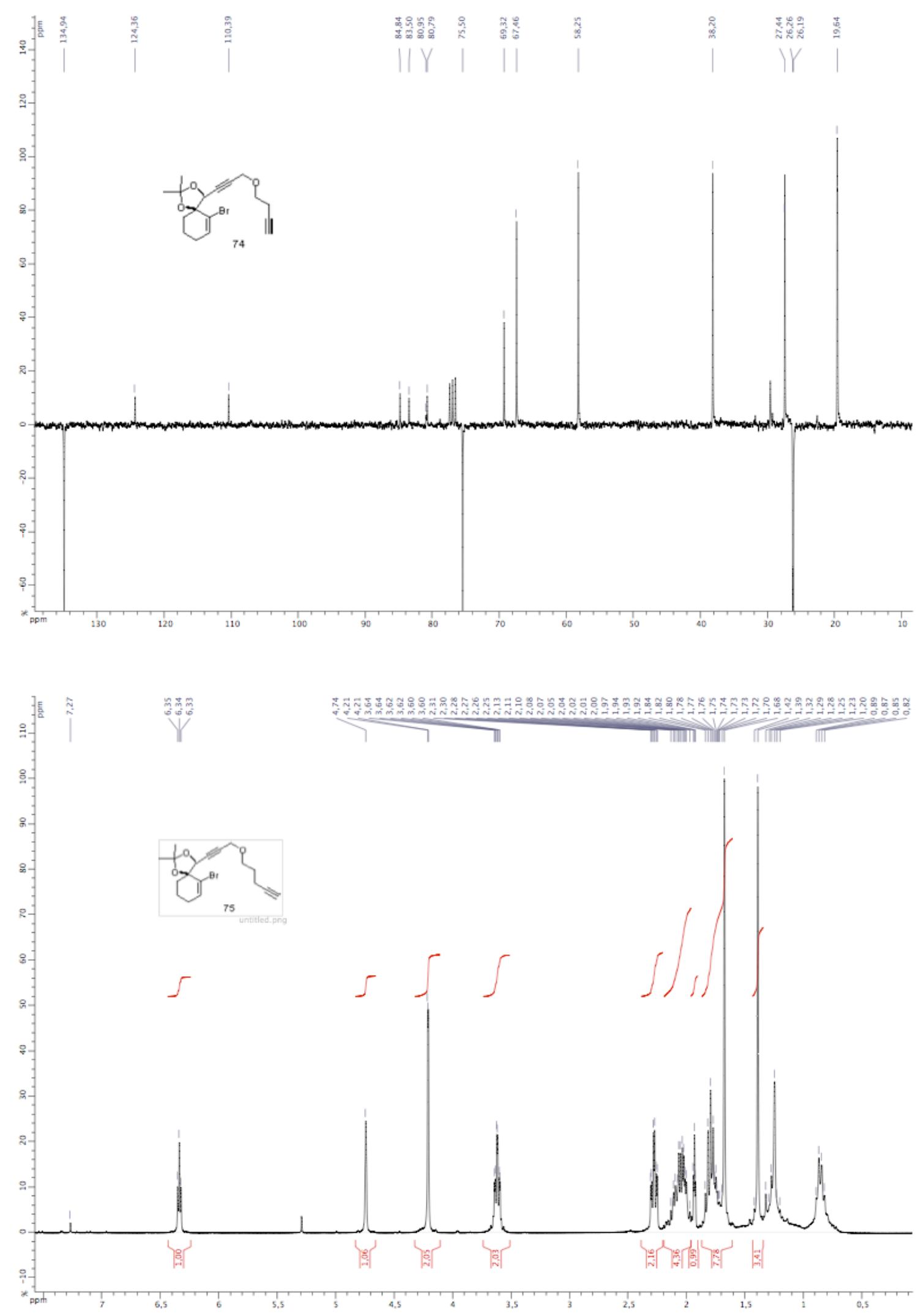

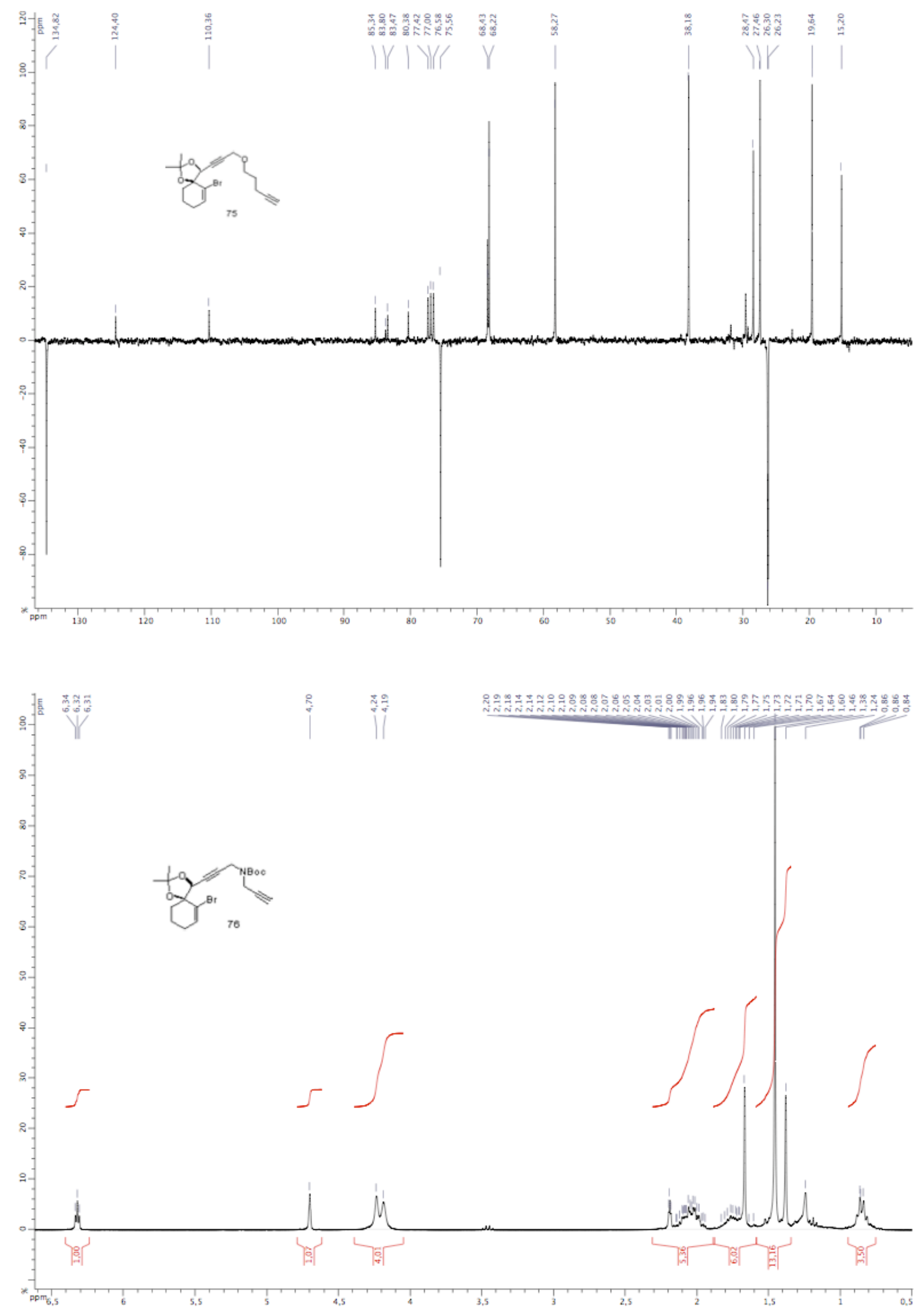

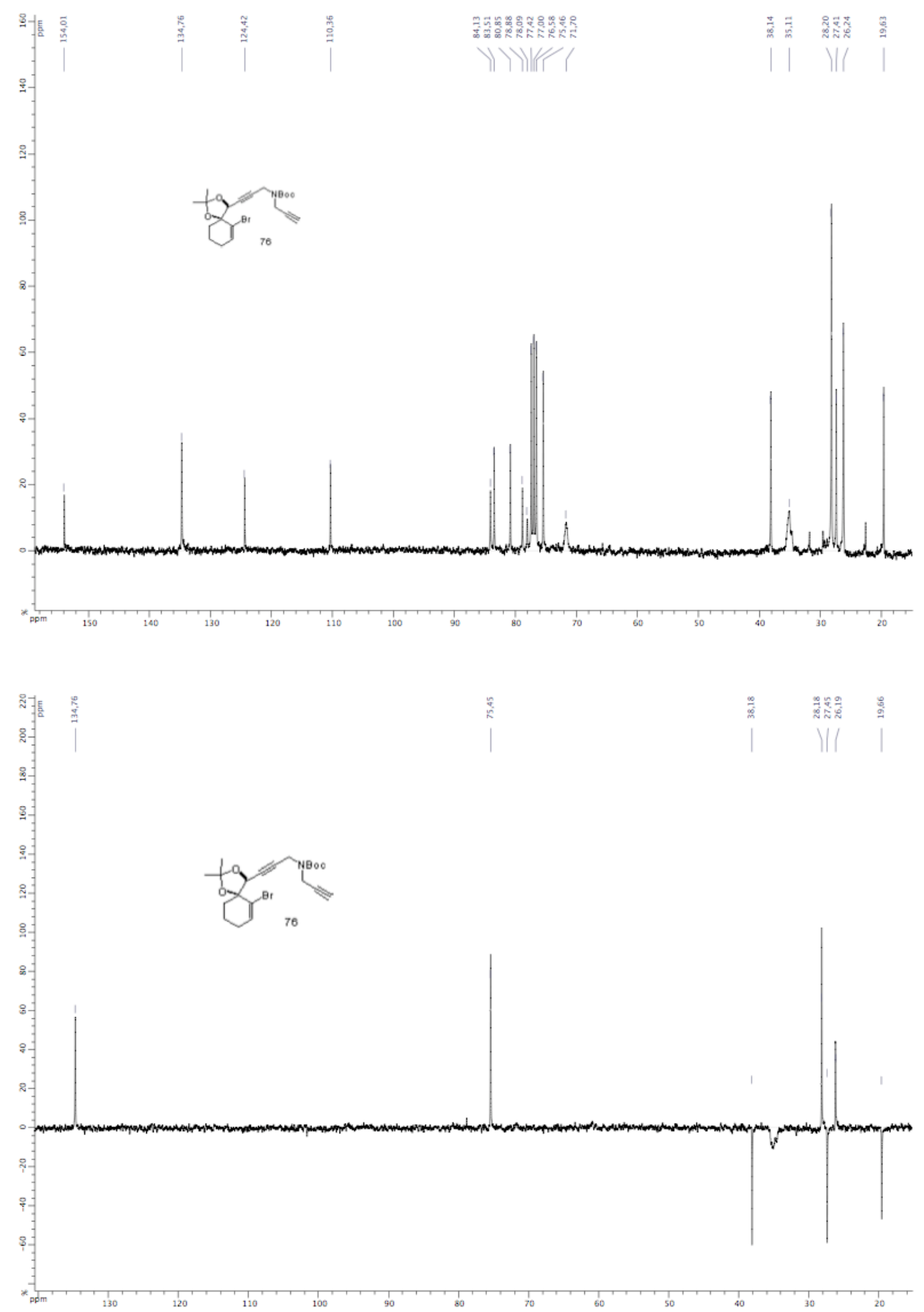

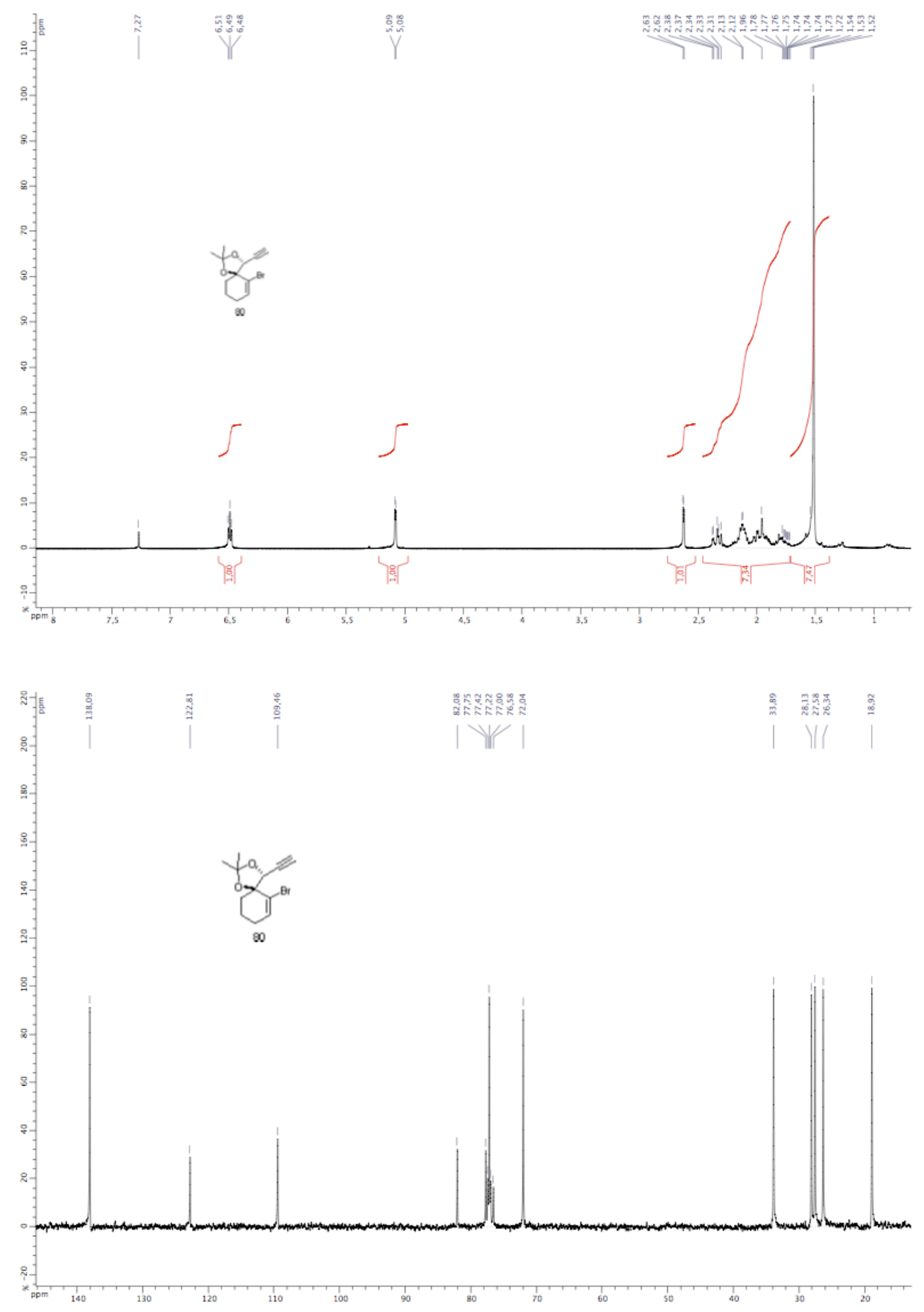

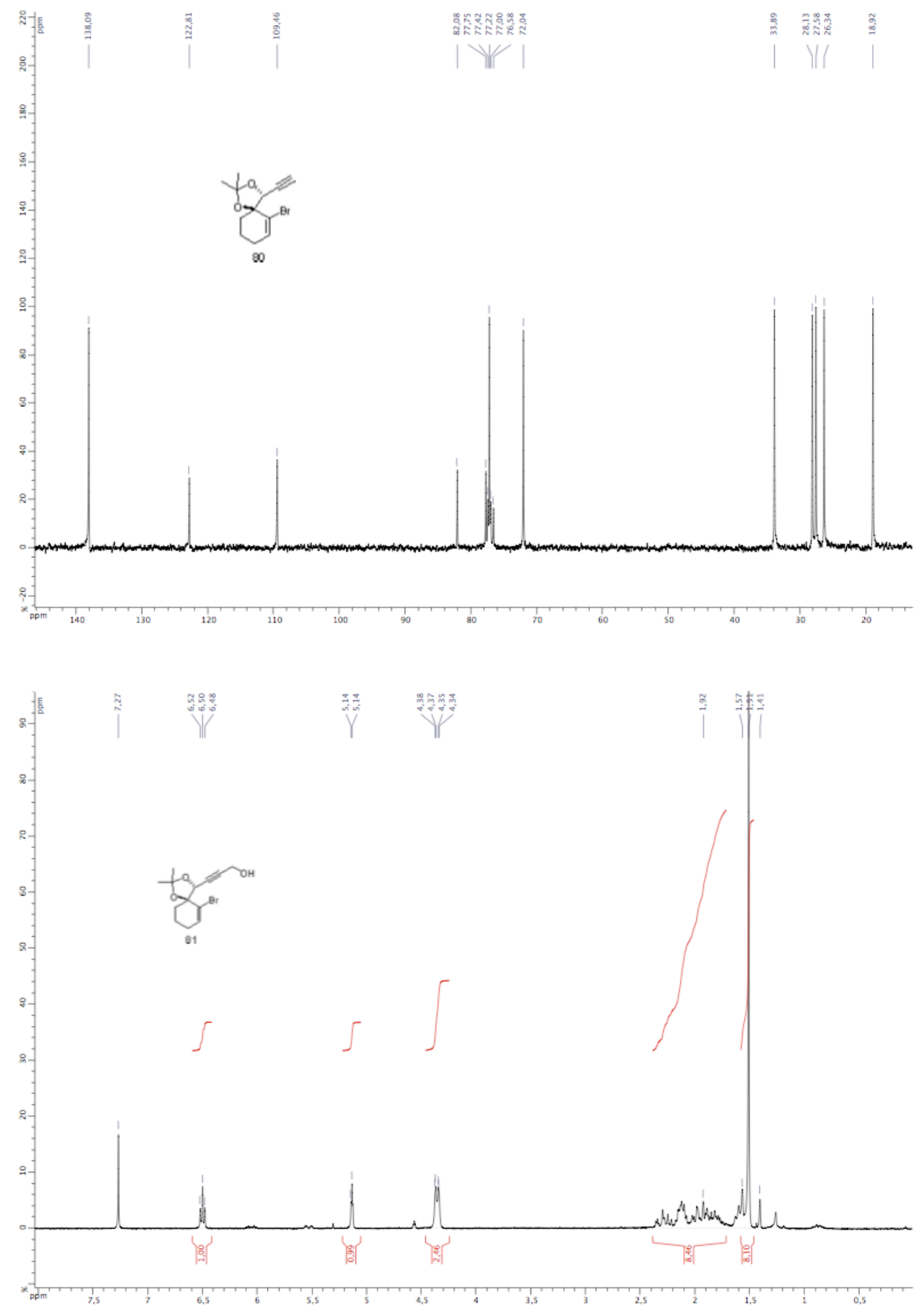

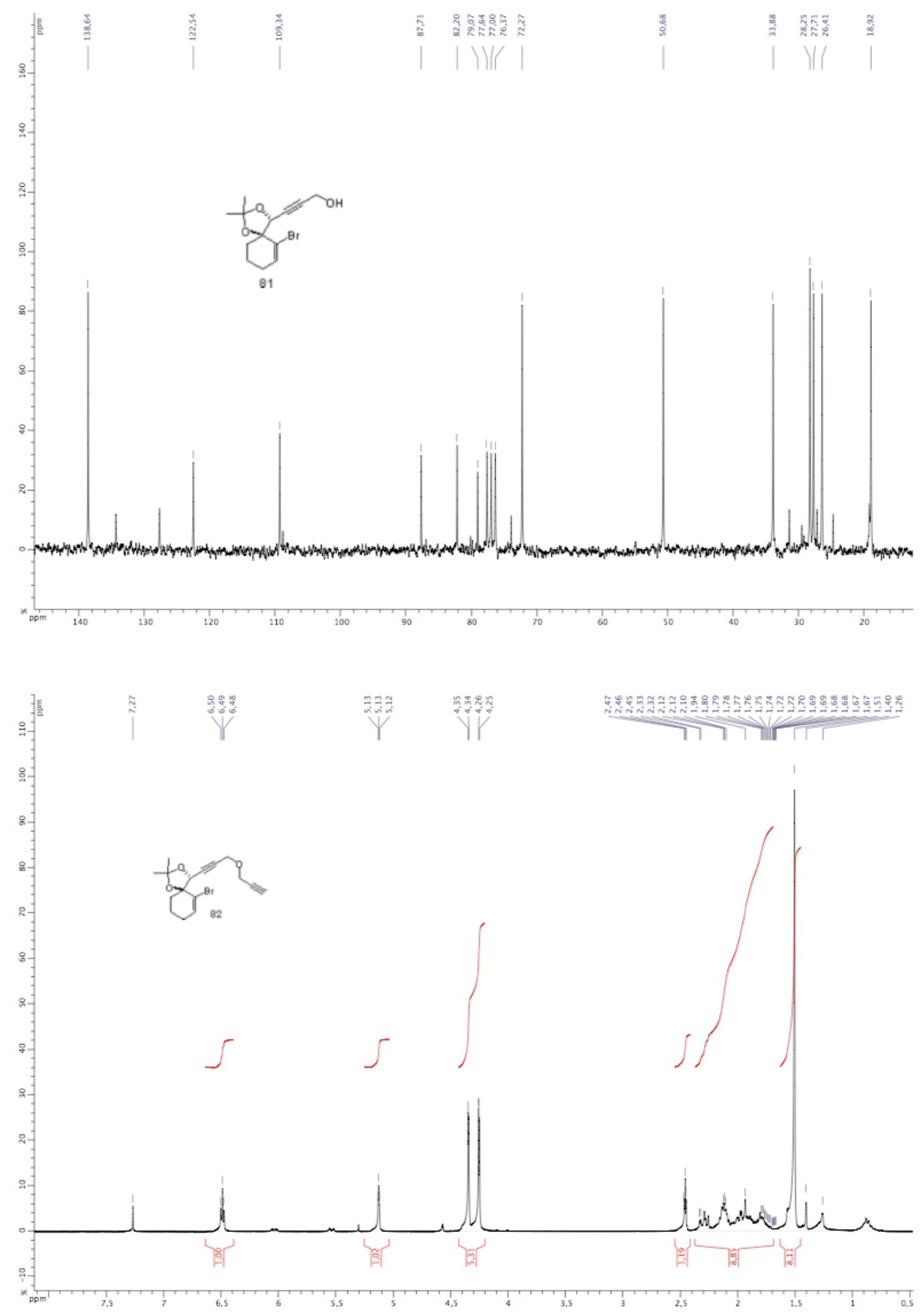

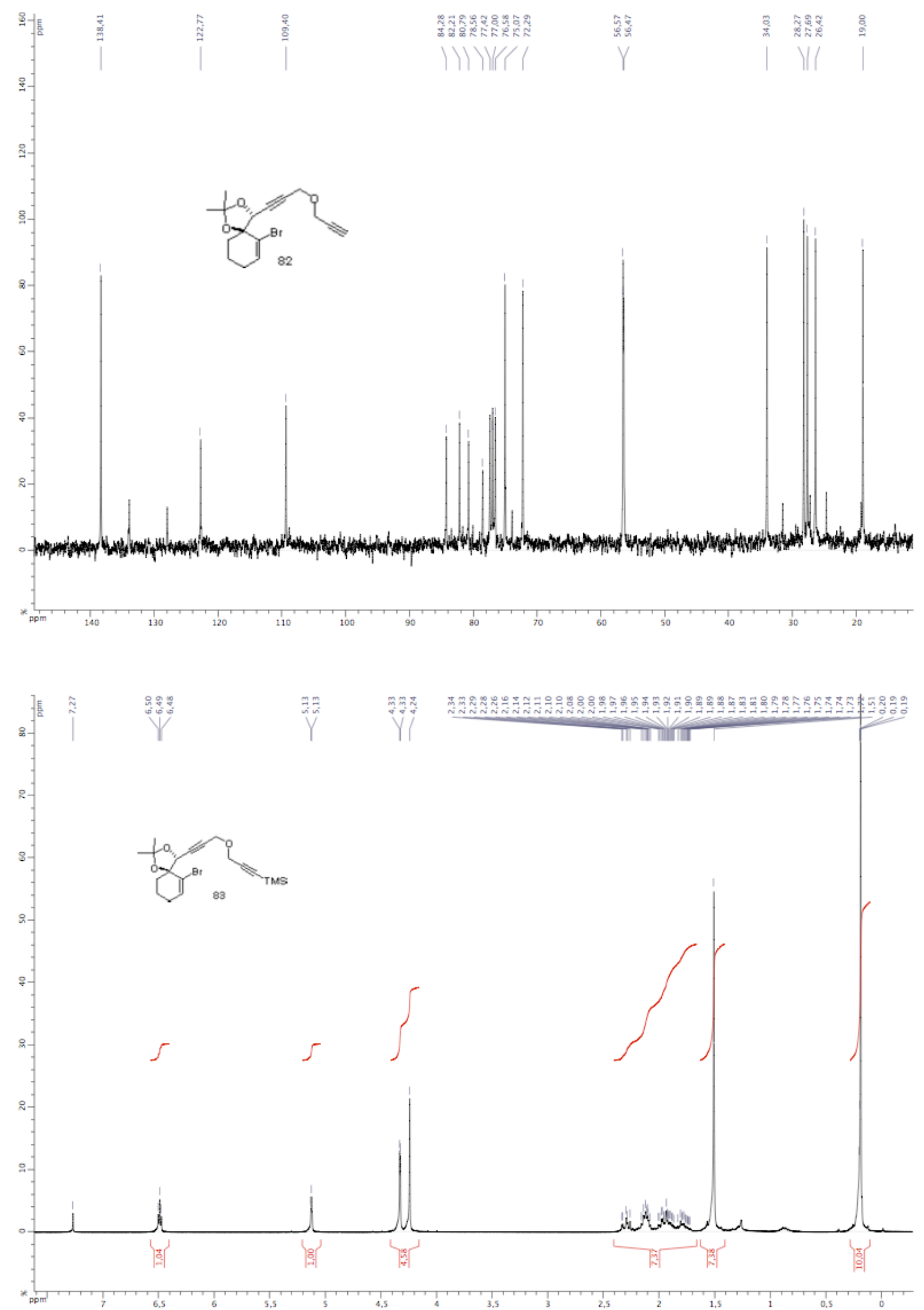

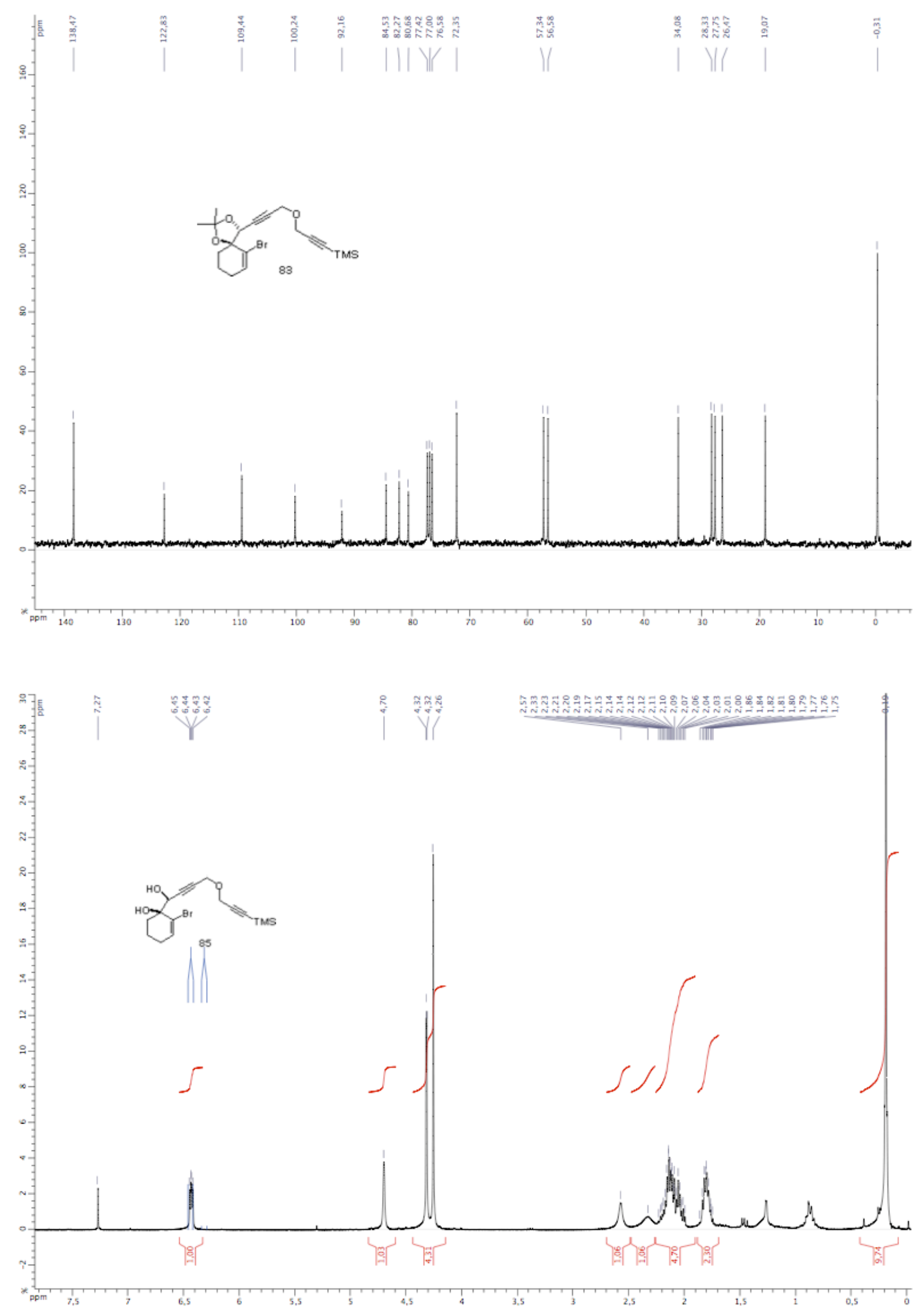

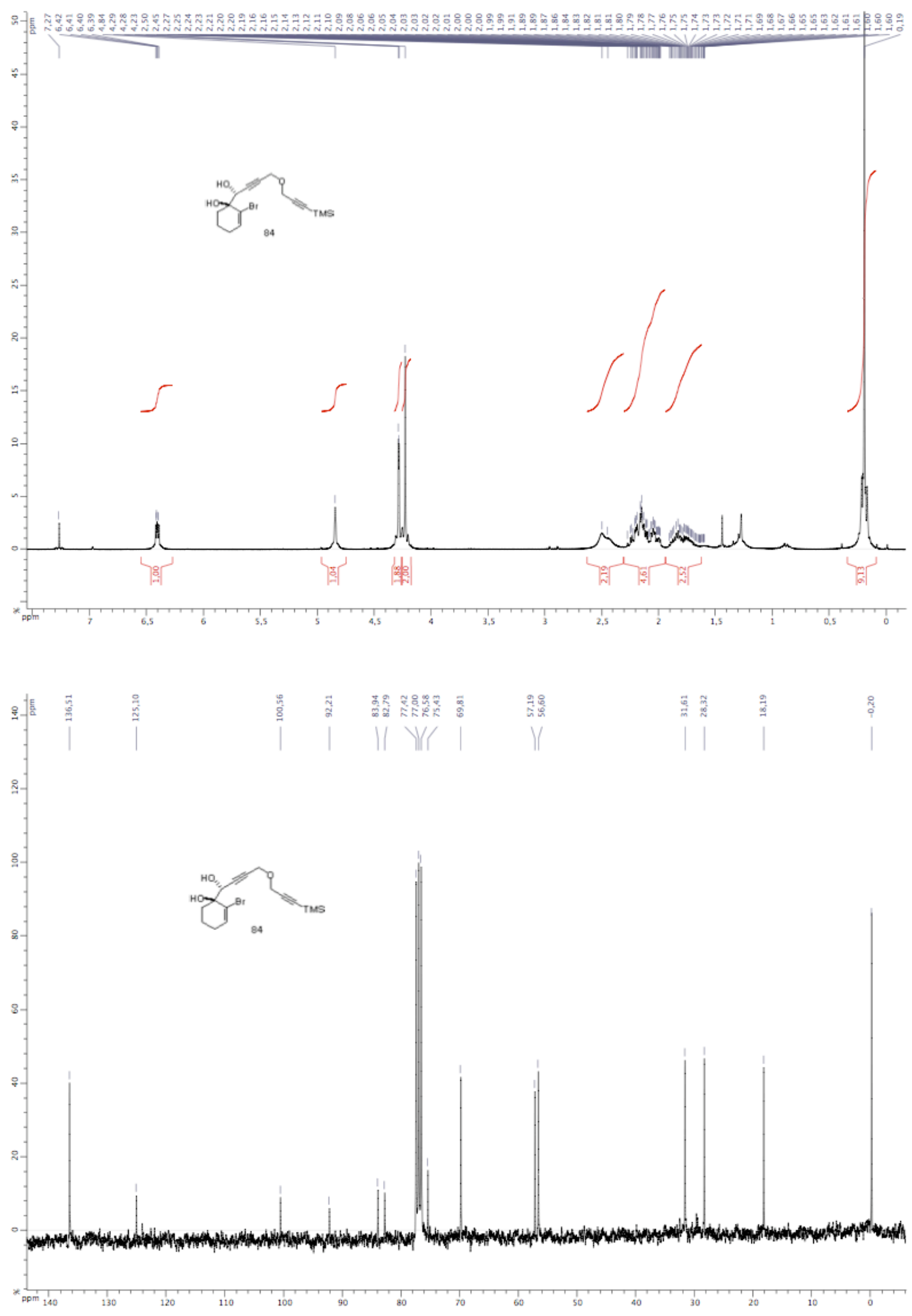

S67 


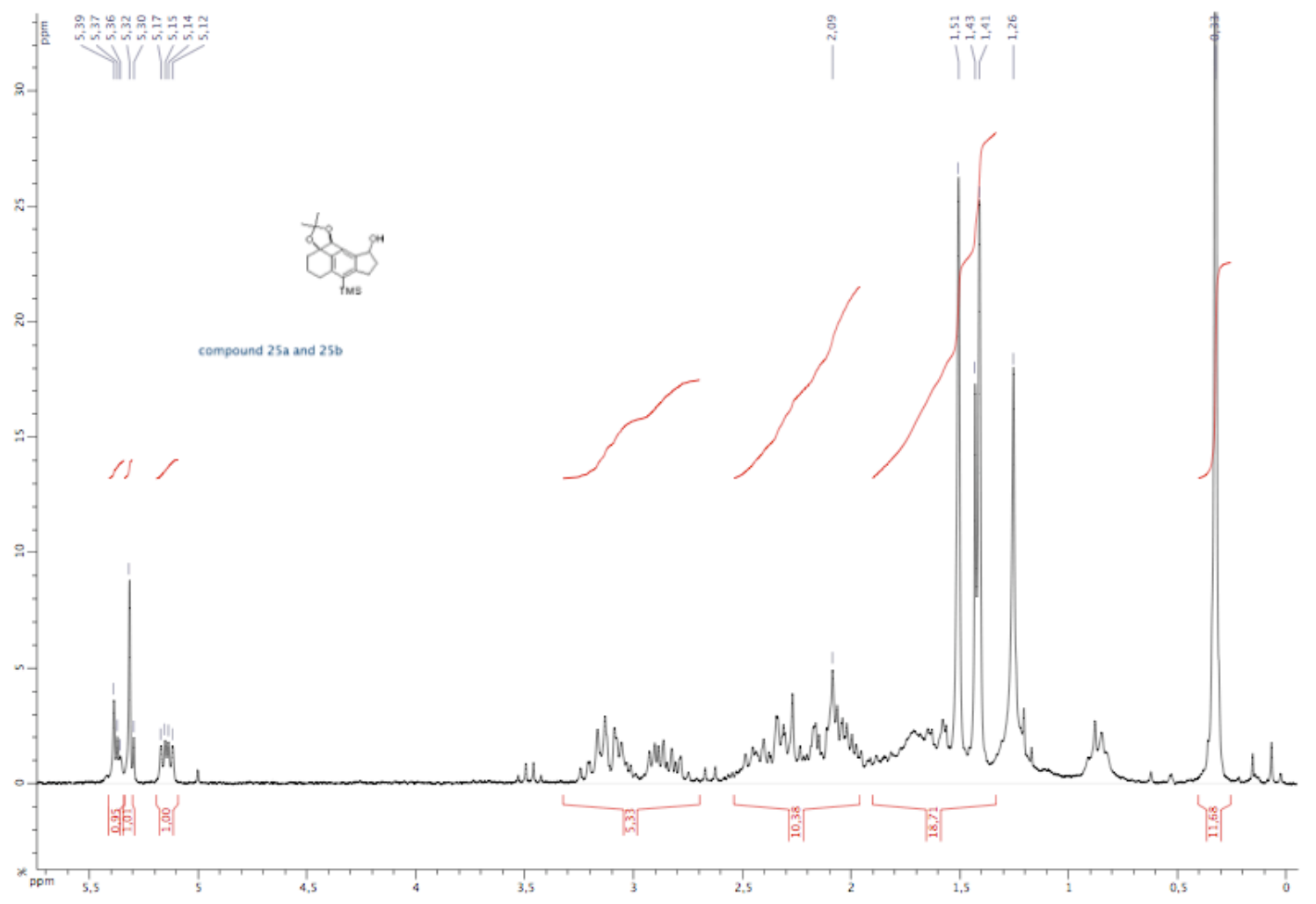

